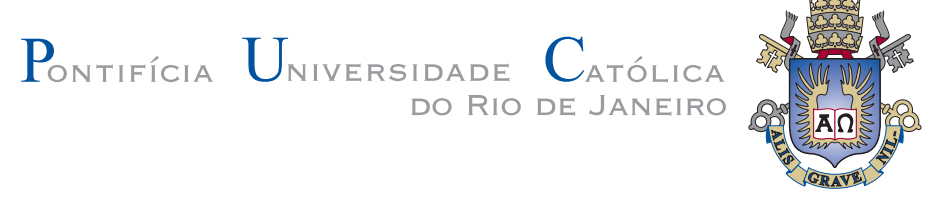

Américo Ariel Rubin de Celis Vidal

\title{
Modelagem Matemática da Interferência Produzida por Redes VSAT/MF-TDMA
}

Tese de Doutorado

Tese apresentada ao Programa de Pós-graduação em Engenharia Elétrica do Departamento de Engenharia Elétrica da PUC-Rio como requisito parcial para obtenção do grau de Doutor em Engenharia Elétrica.

Orientador: Prof. José Mauro Pedro Fortes 


\title{
Américo Ariel Rubin de Celis Vidal
}

\section{Modelagem Matemática da Interferência Produzida por Redes VSAT/MF-TDMA}

Tese apresentada ao Programa de Pós-graduação em Engenharia Elétrica do Departamento de Engenharia Elétrica do Centro Técnico Cientifico da PUC-Rio como requisito parcial para obtenção do grau de Doutor em Engenharia Elétrica. Aprovada pela Comissão Examinadora abaixo assinada.

\author{
Prof. José Mauro Pedro Fortes \\ Orientador \\ Departamento de Engenharia Elétrica - PUC-Rio \\ Prof. Raimundo Sampaio Neto \\ Centro de Estudos em Telecomunicações - PUC-Rio
}

Prof. Marco Antonio Grivet Mattoso Maia

Centro de Estudos em Telecomunicações - PUC-Rio

Prof. Ernesto Leite Pinto

IME

\section{Prof. Pedro Henrique Gouveia Coelho \\ UERJ}

Prof. Paulo Roberto Rosa Lopes Nunes

IME

Prof. Márcio da Silveira Carvalho

Coordenador Setorial do Centro

Técnico Cientifico - PUC-Rio

Rio de Janeiro, 03 de Abril de 2017 
Todos os direitos reservados. É proibida a reprodução total ou parcial do trabalho sem autorização da universidade, do autor e do orientador.

\section{Américo Ariel Rubin de Celis Vidal}

Graduou-se em Engenharia Eletrônica pela Universidad Privada de Tacna - Perú. Recebeu o título de Mestre em Engenharia Elétrica na area de Sistemas de Comunicações pela PUC-Rio em 2012.

Ficha Catalográfica

Rubin de Celis Vidal, Américo Ariel

Modelagem Matemática da Interferência Produzida por Redes VSAT/MF-TDMA/ Américo Ariel Rubin de Celis Vidal; orientador: José Mauro Pedro Fortes. - Rio de Janeiro : PUC-Rio, Departamento de Engenharia Elétrica, 2017.

111 f: il.(color.) ; $30 \mathrm{~cm}$

1. Tese (doutorado) - Pontifícia Universidade Católica do Rio de Janeiro, Departamento de Engenharia Elétrica.

Inclui bibliografia

1. Engenharia Elétrica - Tese. 2. Comunicações. 3. Satélites 4. GSO-FSS. 5. Interferências. 6. razão portadoraruído. 7. VSAT. 8. Inbound. 9. MF-TDMA. I. Fortes, José Mauro Pedro. II. Pontifícia Universidade Católica do Rio de Janeiro. Departamento de Engenharia Elétrica. III. Título. 
Ao meu pai Aladino e minha mãe Rosa, alicerces da minha vida e meus sonhos. 


\section{Agradecimentos}

Considero que seria difícil e quasi impossível tentar descrever, em tão pouco espaço a importância que minha família e especialmente meus pais Aladino e Rosa, tiveram na minha formação pessoal e acadêmica, e que foram fundamentais na consecução de meus objetivos, permitindo-me fechar mais uma etapa em minha formação professional. A eles, devo praticamente tudo.

Quero aproveitar este espaço para agradecer eternamente a uma pessoa extraordinária, meu orientador o Professor José Mauro Pedro Fortes, por seu apoio, orientação, incentivo, paciência e dedicação especialmente na edição e correção de cada projeto de pesquisa empreendido ao longo destes anos de convivência acadêmica no programa de post graduação da PUC-Rio.

Um agradecimento especial à operadora de satélites Embratel Star One, através de seu Gerente de Engenharia Antônio Paolino Iannelli, e do Engenheiro Carlos Alberto da Costa Rebello pelo fornecimento de dados e parâmetros técnicos das redes VSAT, que contribuíram na análises e validação dos resultados neste trabalho de Tese.

Aos professores do Centro de Estudos em Telecomunicações (CETUC) e do Departamento de Engenharia Elétrica da PUC-Rio, que contribuirão na minha formação acadêmica e especialmente aos professores Raimundo Sampaio Neto e Weiler Alves Finamore pelos conhecimentos ministrados.

Não poderia deixar de agradecer a minha avó Maximiliana, minhas irmãs Graciela e Marcia, meus irmãos Jimmy e Milton pelo incondicional apoio sempre mostrado. A minhas sobrinhas: Linda, Wendy, Stephanie e Nigella e sobrinhos Yonathan,Wilfredo e Piero.

Também gostaria de agradecer aos meus amigos Ivan e Carlos Aguilar, meus colegas do CETUC e da PUC-Rio: Franklin Sanchez, Gustavo Bustamante, Harry Anacleto, Alexander Hilario, Walter Aliaga, Uwe Rojas, Alfredo Cordova, Alberth Tamo, Leonel Arévalo, Juliana Carvalho, Jennifer Mendez, Diego Villafani, Maurício Nava, César A. Sierra, Jussif Abularach, Christian Arcos, Jose Calpa, Hugo e Victor Ayma, Darwin Pereira, Teddy Surco, Keyla Mora, Marcelo Molina, Juan Pablo Mayoral, Lisseth Saavedra, Luis Ynoquio, Leonardo Leyva, Rainel Sánchez, Dailys Arronde, Gidy Florez, Carlos Orihuela, Tamara Guerra, Amanda Motta, Isabela Maia, Kinko Mabamba Linden e Andy Alvarez, pelo apoio, sugestões e interesse no desenvolvimento deste trabalho. E aos amigos e funcionários do Departamento de Engenharia Elétrica da PUC-Rio, em especial ao pessoal do CETUC, pela simpatia e disponibilidade para toda e qualquer ajuda.

Finalmente, gostaria de agradecer ao Governo Brasileiro, à Pontifícia Universidade Católica de Rio de Janeiro (PUC-Rio), e o apoio financeiro provido pelo Conselho Nacional de Desenvolvimento Cientifico e Tecnológico - CNPq sem os quais este trabalho não poderia ter sido realizado. 


\section{Resumo}

Rubin de Celis Vidal, Américo Ariel; Fortes, José Mauro Pedro. Modelagem Matemática da Interferência Produzida por Redes VSAT/MF-TDMA. Rio de Janeiro, 2017. 111p. Tese de Doutorado Departamento de Engenharia Elétrica, Pontifícia Universidade Católica do Rio de Janeiro.

Neste trabalho é desenvolvido um modelo matemático para descrever o comportamento estatístico da interferência produzida por redes VSAT/MFTDMA. O modelo proposto é utilizado para avaliar a interferência produzida pelos lances de subida de enlaces VSAT/MF-TDMA em enlaces de uma outra rede que utiliza um satélite vizinho. No modelo proposto, expressões analíticas foram desenvolvidas para levar em conta os efeitos de variações nas potências transmitidas, nos tamanhos das antenas e nos erros de apontamento das antenas transmissoras. As posições geográficas das estações terrenas são modeladas por processos pontuais de Poisson, bi-dimensionais. O modelo proposto é suficientemente geral para acomodar outros tipos de processos pontuais, além de situações envolvendo áreas de serviço contendo múltiplos tipos de distribuição geográfica das estações terrenas. Resultados numéricos obtidos com o modelo proposto são comparados àqueles baseados em valores reais de parâmetros (e.g. localização das estações terrenas, tamanhos de antenas e potências de transmissão) que foram fornecidos por um operador brasileiro de satélites .

\section{Palavras-chave}

Comunicações por satélites; GSO-FSS; Interferências; razão portadoraruído; Redes VSAT/MF-TDMA. 


\section{Abstract}

Rubin de Celis Vidal, Américo Ariel; Fortes, José Mauro Pedro(Advisor). Mathematical modelling of the interference produced by VSAT/MF-TDMA satellite networks. Rio de Janeiro, 2017. 111p. Tese Doutorado - Departamento de Engenharia Elétrica, Pontifícia Universidade Católica do Rio de Janeiro.

In this work a mathematical model to describe the statistical behavior of the interference produced by VSAT/MF-TDMA networks is developed. The model is used to assess the interference produced by the uplinks of a VSAT/MF-TDMA network into links of a network that uses a neighboring satellite. In the proposed model, analytical expressions were developed to account for the effects of the varying transmitting powers, antenna sizes, and transmitting antenna pointing errors. The earth station locations are modeled by a two dimensional Poisson point process. The model is general enough to accommodate other types of point processes and can be applied to situations involving service areas containing multiple types of earth station geographical distribution. Numerical results obtained with the proposed model are compared to those based on the actual parameters values (e.g. earth station locations, antenna sizes and transmitting powers) which were provided by a Brazilian satellite operator.

\section{Keywords}

Satellite Communications; GSO-FSS; Interference; carrier-to-noise ratio; VSAT/MF-TDMA Networks. 


\section{Sumário}

1 Introdução 14

2 Descrição do Problema 17

3 Modelagem Matemática $\quad 21$

3.1 Considerações básicas 21

3.2 Determinação da função densidade de probabilidade da densidade de potência interferente $p_{i}(I) \quad 23$

3.3 Expressão Analítica da Função $H_{\mathbf{R} j}(I) \quad 25$

3.3.1 Caso particular: densidade de e.i.r.p. estatisticamente independente da posição do terminal VSAT 28

3.4 Consideração de regiões contendo diferentes características de distribuição de terminais

3.4.1 Caso particular: densidade de e.i.r.p. estatisticamente independente da posição do terminal VSAT, em cada região $\mathcal{R}_{m} \quad 30$

4 Resultados Numéricos 33

4.1 Considerações específicas sobre os cenários analisados neste capítulo 34

4.1.1 Identificação de regiões 35

4.1.2 Atenuação por chuva e tamanhos de antenas 37

4.1.3 Determinação da densidade de e.i.r.p. associada a cada tamanho de antena 38

4.1.4 Distribuição de terminais VSAT/MF-TDMA 39

4.1.5 Posições orbitais e antenas 41

4.2 Alguns exemplos específicos 45

4.2.1 Regiões com um único tipo de distribuição de terminais 45

4.2.2 Regiões com múltiplas distribuições de terminais 61

5 Conclusões e sugestões para trabalhos futuros. $\quad 71$

$\begin{array}{lll}5.1 \text { Conclusão } & 71\end{array}$

$\begin{array}{ll}5.2 & \text { Sugestões para trabalhos futuros. }\end{array}$

$\begin{array}{ll}\text { Referências bibliográficas } & \mathbf{7 4}\end{array}$

$\begin{array}{ll}\text { A Geometria Estocástica } & 78\end{array}$

$\begin{array}{lll}\text { A.1 Processo pontual Binomial } & 78\end{array}$

$\begin{array}{ll}\text { A.2 Processo pontual Poisson Homogêneo } & 80\end{array}$

A.3 Processo pontual Poisson Geral e Não Homogêneo 81

A.4 Modelos de Processos Pontuais $\quad 82$

A.4.1 Operações sobre Processos Pontuais 82

A.5 Processos Cox 85

A.6 Processos Neyman-Scott 86

A.7 Processos Gibss $\quad 86$

$\begin{array}{ll}\text { B Simulações de Processos Pontuais } & 88\end{array}$ 
B.1 Simulação do processo pontual Binomial $\quad 88$

B.2 Simulação do processo pontual Poisson homogêneo $\quad 89$

B.3 Simulação do Processo pontual Poisson Geral Não Homogêneo 90

B.4 Simulação do Processo Pontual de Poisson (PPP) homogéneo sobre as regiões geográficas definidas na Subseção 4.1.1 90

C Diagrama de radiação das antenas correspondentes aos terminais interferentes $T^{\prime}$ da rede VSAT: Recomendação ITU-R F.1245-1

D Diagrama de radiação da antena receptora do satélite vitima $S$ : Recomendação ITU-R S.672-4

E Níveis máximos admissíveis da densidade e.i.r.p., das antenas transmissoras VSAT, Recomendação ITU-R S.728-1

F Comportamento estatístico da Função distribuição de probabilidade complementar correspondente por região $\mathcal{R}_{m},(m=1 \ldots 6)$, para a rede $1 \mathrm{e}$ 2 com $R_{\text {ref }}\left(54,90^{\circ} \mathrm{W}, 9,70^{\circ} \mathrm{S}\right)$

F.1 Comparação do comportamento estatístico da variável aleatória $i_{n}$ obtida a partir do modelo proposto e estimativas a partir de dados reais da operadora de satélites Embratel Star One, por região $\mathcal{R}_{m},(m=$ $1 \ldots 6)$ correspondente à Rede 1 de 211 terminais VSAT.

F.2 Comparação do comportamento estatístico da variável aleatória $i_{n}$ obtida a partir do modelo proposto e estimativas a partir de dados reais da operadora de satélites Embratel Star One, por região $\mathcal{R}_{m},(m=$ $1 \ldots 6)$ correspondente à Rede 2 de 78 terminais VSAT. 


\section{Lista de figuras}

2.1 Direção dos enlaces inbound e outbound, em uma rede VSAT (Very Small Aperture Terminal)

2.2 Cenário correspondente ao "padrão de interferências" observado no receptor da rede vítima devido à transmissão dos terminais $T_{1}^{\prime}, \ldots, T_{N}^{\prime}$ de uma rede VSAT/MF-TDMA.

3.1 Geometria associada ao modelo.

3.2 Diagrama de radiação usual da antena do terminal interferente $T^{\prime}$.

3.3 Dependência de $\theta_{\mathbf{r}}$ sobre os erros de apontamento da antena em azimute e elevação.

4.1 Mapa da densidade demográfica do Brasil [Habitantes $/ \mathrm{km}^{2}$ ]. Fonte: Instituto Brasileiro de Geografia e Estatística (IBGE)

4.2 Taxa de precipitação pluvial no território brasileiro $[\mathrm{mm} / \mathrm{h}]$.

4.3 Taxa de precipitação pluvial $[\mathrm{mm} / \mathrm{h}]$ e Densidade demográfica [Habitantes por $\mathrm{km}^{2}$ ] do Brasil .

4.4 Separação orbital $\Delta$ (graus), entre satélites interferente $S^{\prime}$ e vítima $S$.

4.5 Diagrama de radiação da antena receptora do satélite vítima $S$.

4.6 Contornos de cobertura da antena receptora do satélite vítima.

4.7 Diagrama de radiação das antenas transmissoras dos terminais VSAT. 44

4.8 Amostra típica da distribuição de terminais da Rede 1 na Região 1 (PPP com densidade média de $8,96 \times 10^{-5}$ terminais por $\mathrm{km}^{2}$ ). Inclui a posição geográfica $R_{r e f}$ utilizada no cálculo do valor de referência $i_{\text {ref }}\left(44,96^{\circ} \mathrm{W}, 19,01^{\circ} \mathrm{S}\right)$.

4.9 Amostra típica da distribuição de terminais da Rede 2 na Região 1 (PPP com densidade média de $2,40 \times 10^{-5}$ terminais por $\mathrm{km}^{2}$ ). Inclui a posição geográfica $R_{\text {ref }}$ utilizada no cálculo do valor de referência $i_{\text {ref }}\left(44,96^{\circ} \mathrm{W}, 19,01^{\circ} \mathrm{S}\right)$.

4.10 Função distribuição de probabilidade complementar correspondente à Rede 1, com 67 terminais VSAT na Região 1.

4.11 Função distribuição de probabilidade complementar correspondente à Rede 2, com 18 terminais VSAT na Região 1.

4.12 Amostra típica da distribuição de terminais da Rede 1 na Região 6 (PPP com densidade média de $1,66 \times 10^{-5}$ terminais por $\mathrm{km}^{2}$ ). Inclui a posição geográfica $R_{\text {ref }}$ utilizada no cálculo do valor de referência $i_{\text {ref }}\left(54,90^{\circ} \mathrm{W}, 11,70^{\circ} \mathrm{S}\right)$.

4.13 Amostra típica da distribuição de terminais da Rede 2 na Região 6 (PPP com densidade média de $5,09 \times 10^{-6}$ terminais por $\mathrm{km}^{2}$ ). Inclui a posição geográfica $R_{r e f}$ utilizada no cálculo do valor de referência $i_{\text {ref }}\left(54,90^{\circ} \mathrm{W}, 11,70^{\circ} \mathrm{S}\right)$.

4.14 Função distribuição de probabilidade complementar correspondente à Rede 1, com 85 terminais VSAT na Região 6.

4.15 Função distribuição de probabilidade complementar correspondente à Rede 2, com 26 terminais VSAT na Região 6. 
4.16 Amostra típica da distribuição de terminais da Rede 1 na Região 4 (PPP com densidade média de $1,516 \times 10^{-5}$ terminais por $\mathrm{km}^{2}$ ). Inclui a posição geográfica $R_{\text {ref }}$ utilizada no cálculo do valor de referência $i_{\text {ref }}\left(52,31^{\circ} \mathrm{W}, 7,76^{\circ} \mathrm{S}\right)$.

4.17 Amostra típica da distribuição de terminais da Rede 2 na Região 4 (PPP com densidade média de $1,241 \times 10^{-5}$ terminais por $\mathrm{km}^{2}$ ). Inclui a posição geográfica $R_{\text {ref }}$ utilizada no cálculo do valor de referência $i_{\text {ref }}\left(52,31^{\circ} \mathrm{W}, 7,76^{\circ} \mathrm{S}\right)$.

4.18 Função distribuição de probabilidade complementar correspondente à Rede 1 , com 11 terminais VSAT na Região 4.

4.19 Função distribuição de probabilidade complementar correspondente à Rede 2, com 9 terminais VSAT na Região 4.

4.20 Amostras típicas das distribuições de terminais correspondentes a cada região da Rede 1 no território brasileiro (PPP com densidades médias por região [terminais $/ \mathrm{km}^{2}$ ], apresentada na Tabela 4.7), com $R_{\text {ref }}\left(54,90^{\circ} \mathrm{W}, 9,70^{\circ} \mathrm{S}\right)$.

4.21 Amostras típicas das distribuições de terminais correspondentes a cada região da Rede 2 no território brasileiro (PPP com densidade média por região [terminais $/ \mathrm{km}^{2}$ ], apresentada na Tabela 4.8), com $R_{r e f}\left(54,90^{\circ} \mathrm{W}, 9,70^{\circ} \mathrm{S}\right)$.

4.22 Função distribuição de probabilidade complementar correspondente à região $\mathcal{R}_{1}, \mathcal{R}_{2}, \mathcal{R}_{3}, \mathcal{R}_{4}, \mathcal{R}_{5}$, e $\mathcal{R}_{6}$ da Rede 1 , com $R_{\text {ref }}$ $\left(54,90^{\circ} \mathrm{W}, 9,70^{\circ} \mathrm{S}\right)$.

4.23 Função distribuição de probabilidade complementar correspondente à região $\mathcal{R}_{1}, \mathcal{R}_{2}, \mathcal{R}_{3}, \mathcal{R}_{4}, \mathcal{R}_{5}$, e $\mathcal{R}_{6}$ da Rede 2 , com $R_{\text {ref }}$ $\left(54,90^{\circ} \mathrm{W}, 9,70^{\circ} \mathrm{S}\right)$.

4.24 Função distribuição de probabilidade complementar correspondente à Rede 1 , com 211 terminais VSAT, com $R_{\text {ref }}\left(54,90^{\circ} \mathrm{W}, 9,70^{\circ} \mathrm{S}\right)$.

4.25 Função distribuição de probabilidade complementar correspondente à Rede 2, com 78 terminais VSAT, com $R_{\text {ref }}\left(54,90^{\circ} \mathrm{W}, 9,70^{\circ} \mathrm{S}\right)$.

A.1 Exemplo correspondente a um processo pontual Binomial, em $W=[0,1]^{2}$ com $n=100$ pontos

A.2 Exemplo: Processo Pontual de Poisson como função intensidade linearmente crescente $\lambda(x)$

A.3 Padrões de pontos Thinning, pontos do padrão original são marcados como + , e pontos excluídos $\oplus$. (a) Thinning Independente, realizado por p-thinning. (b) Thinning dependente, exclusão dos pontos com vizinhos mais próximos, com distância $R$.

A.4 Exemplo de um processo pontual cluster. Pontos pai são marcados por $\circ$ e pontos filhos por $\bullet$.

B.1 Simulação de 45 pontos aleatórios uniformemente distribuídos em um região $W$.

B.2 Simulação de um Processo pontual de Poisson(PPP), uniformemente distribuído correspondente à Região 1.

B.3 Simulação de um Processo pontual de Poisson(PPP), uniformemente distribuído correspondente à Região 6 . 
C.1 Diagramas de radiação das antenas dos terminais interferentes $T^{\prime}$, da rede VSAT MF-TDMA.

D.1 Diagrama de radiação da antena receptora do satélite vítima $S . \quad 96$

E.1 Diagrama de radiação antena 0,96 m. e nível máximo admissível da densidade e.i.r.p.e (Recomendação ITU-R S.728-1) em dB(W/Hz) 98

E.2 Diagrama de radiação antena $1,20 \mathrm{~m}$. e nível máximo admissível da densidade e.i.r.p.e (Recomendação ITU-R S.728-1) em dB(W/Hz) 99

E.3 Diagrama de radiação antena 1,80 m. e nível máximo admissível da densidade e.i.r.p.e (Recomendação ITU-R S.728-1) em dB(W/Hz) 99

F.1 Número de terminais $\mathcal{N}_{m}: 84$.

F.2 Número de terminais $\mathcal{N}_{m}: 7 . \quad 101$

F.3 Número de terminais $\mathcal{N}_{m}: 65$. 102

F.4 Número de terminais $\mathcal{N}_{m}: 9$.

F.5 Número de terminais $\mathcal{N}_{m}: 10 . \quad 104$

F.6 Número de terminais $\mathcal{N}_{m}: 36.105$

F.7 Número de terminais $\mathcal{N}_{m}: 31$.

F.8 Número de terminais $\mathcal{N}_{m}: 3 . \quad 107$

F.9 Número de terminais $\mathcal{N}_{m}: 24.108$

F.10 Número de terminais $\mathcal{N}_{m}: 3 . \quad 109$

F.11 Número de terminais $\mathcal{N}_{m}: 4$.

F.12 Número de terminais $\mathcal{N}_{m}: 13$. 


\section{Lista de tabelas}

4.1 Taxa de precipitação e tamanho de antena por região

4.2 Valores de densidade de e.i.r.p., transmitidos pelos terminais VSAT na direção de máxima radiação das antenas.

4.3 Valores de $N_{m}$ e $E_{j m}$, correspondentes utilizados em cada uma das seis regiões geográficas ilustradas na Figura 4.3.

4.4 População $\mathcal{P}_{m}$ (habitantes) e Área $\mathcal{A}_{m}\left(\mathrm{~km}^{2}\right)$ correspondentes a cada região $\mathcal{R}_{m}$.

4.5 Densidade de terminais por região, para uma rede VSAT (inbound), com $\mathcal{N}_{T}$ terminais.

4.6 Valores de $G_{\max }, d / \lambda$ e $\varphi_{m}$ para antenas de diferentes diâmetros 44

4.7 Número $\mathcal{N}_{m}$ e densidade $\lambda_{m}$ de terminais de terminais por região (Rede $1-\mathcal{N}_{T}=211$ ).

4.8 Número $\mathcal{N}_{m}$ e densidade $\lambda_{m}$ de terminais de terminais por região (Rede $2-\mathcal{N}_{T}=78$ ).

4.9 Valores de probabilidade $P\left(\mathbf{r} \in \mathcal{R}_{m}\right)$ de cada região $\mathcal{R}_{m}$ ( $m=$ $1, \ldots, 6)$, para a Rede 1 e Rede 2 .

B.1 Parâmetros $R, W$ e $\lambda$. Assumindo uma rede com $\mathcal{N}_{T}=211$ terminais 91

C.1 Parâmetros correspondentes às antenas dos terminais interferentes $T^{\prime}$ VSAT.

D.1 Parâmetros da antena receptora do satélite $S$. 


\section{Introdução}

Nos últimos anos, mais especificamente durante a última década, a demanda por serviços de transferência de dados em altas taxas através de enlaces via satélite tem aumentado significativamente. Atualmente a maioria destes serviços tem como clientes usuários residenciais e empresas de pequeno, médio e grande porte que utilizam redes por satélite com tecnologia VSAT (Very Small Aperture Terminal).

A grande maioria desses sistemas VSAT utiliza o protocolo de múltiplo acesso por divisão no tempo e frequência (multiple frequency - time division multiple access MF-TDMA) em seu enlace inbound. Neste tipo de múltiplo acesso, a portadora recebida no satélite é composta de quadros (TDMA frame) divididos em diversas janelas de tempo (time slots), que são utilizadas por diferentes terminais VSAT para a transmissão de seus dados. Dependendo de sua localização, da cobertura da antena de recepção do satélite e das condições de propagação, estes terminais podem operar com diferentes tamanhos de antenas transmissoras e diferentes níveis de potência de transmissão. Além disso, os terminais de uma rede VSAT operam usualmente sob erros de apontamento de suas antenas transmissoras, que variam, em maior ou menor grau, dependendo do terminal considerado. Todos estes aspectos contribuem para a formação de um "padrão de interferências", que pode afetar enlaces de outras redes como por exemplo, redes que operam com satélites próximos (espaçamento orbital menor que três graus) àquele da rede VSAT.

Uma metodologia desenvolvida para a avaliação das interferências geradas pelos terminais VSAT de redes GSO-FSS (Geostationary Satellite Orbit Fixed Satellite Service), que inclui, além dos terminais fixos, terminais montados sobre plataformas móveis (trens, veículos, navios) é apresentada na Recomendação ITU-R S.2029 do Setor de Radiocomunicações da União Internacional de Telecomunicações [1]. Esta metodologia modela a posição geográfica dos terminais da rede VSAT e a densidade de e.i.r.p. (Effective Isotropic Radiated Power) como variáveis aleatórias, e considera que estas duas variáveis aleatórias são estatisticamente independentes. Esta é uma restrição muito forte do modelo, uma vez que a densidade de e.i.r.p. transmitida por um terminal depende, de certa maneira, de sua localização geográfica. É usual, por exemplo, que terminais localizados nas regiões com altas taxa de precipitação de chuvas utilizem antenas maiores e consequentemente tenham um nível de densidade de e.i.r.p. maior do que os terminais localizados em regiões de pouca chuva. 
Dada a importância da caracterização estatística das interferências geradas por portadoras VSAT/TDMA na análise de seus efeitos sobre o desempenho de outras portadoras que compartilham a mesma faixa de frequências, mesmo após a aprovação da Recomendação ITU-R S.2029, o assunto continuou em pauta no âmbito do Setor de Radiocomunicações da União Internacional de Telecomunicações (ITU-R). Nessa linha de pesquisa, um estudo [2] apresentado em setembro de 2013 ao Grupo de Trabalho 4A da Comissão de Estudos 4 do ITU-R (responsável pelos assuntos relativos ao Serviço Fixo por Satélite) propôs uma metodologia para avaliar a sensibilidade dos níveis de interferência gerados pelas redes VSAT/TDMA à localização geográfica dos terminais VSAT interferentes. Mais especificamente, a metodologia apresentada propõe uma avaliação do efeito da quantização das posições geográficas dos terminais VSAT nos níveis de interferência gerados. Esta avaliação seria feita, via simulação, através da comparação dos resultados (níveis de interferência) obtidos considerando-se a quantização das posições geográficas dos terminais com aqueles obtidos ao se considerar que as posições geográficas dos terminais têm distribuição uniforme ao longo da área de serviço da rede VSAT. O estudo apresentado [1] serviu de base para os estudos e análises desenvolvidos no âmbito do ITU-R entre 2013 e 2015, resultando na aprovação, em junho de 2015, do Relatório ITU-R S.2362-0 [6].

Os estudos realizados no âmbito do ITU-R consideraram, para efeitos da análise, a metodologia proposta em [2]. Assim, foi considerada, uma distribuição uniforme das posições geográficas dos terminais da rede VSAT MFTDMA, sendo analisados os efeitos da quantização dessas posições geográficas na estimação dos níveis de interferência em redes que operam em satélites vizinhos. Além disso, o efeito dos outros parâmetros envolvidos (e.g. potência de transmissão, erros de apontamento), foram avaliados por simulação (Método de Monte Carlo).

Motivado pela indiscutível importância da caracterização estatística das interferências produzidas por redes VSAT/TDMA, este trabalho de tese propõe um modelo matemático para a abordagem do problema, no qual são desenvolvidas expressões analíticas para as funções densidade de probabilidade dos erros de apontamento das antenas dos terminais interferentes e das potências de transmissão desses terminais, consideradas dependentes de suas posições geográficas. No modelo proposto, as posições geográficas dos terminais VSAT são caracterizadas por processos estocásticos pontuais bi-dimensionais (e.g. processos de Poisson, processos binomiais, processos com clusters) que correspondem à área de pesquisa matemática conhecida como Geometria Estocástica [9]. Os resultados obtidos com o modelo proposto são comparados àqueles pro- 
duzidos com base em dados reais fornecidos pela operadora de satélites Embratel Star One.

Assim, no Capítulo 2, é feita uma abordagem sucinta dos conceitos e parâmetros mais importantes relativos aos dois tipos de degradação abordados neste trabalho: a degradação produzida por chuvas e a degradação causada por sinais interferentes gerados por outros sistemas. Esta última, de grande importância, é modelada matematicamente, no Capítulo 3. Assim, no Capítulo 3, um modelo matemático para a caracterização do comportamento estatístico da potência interferente é apresentado, sendo obtidas expressões analíticas para a função densidade de probabilidade da densidade de potência interferente considerando-se a dependência entre a densidade de e.i.r.p. e a posição geográfica do terminal VSAT, além de diferentes tipos de distribuição de terminais. Os resultados são também particularizados para o caso em que a densidade de e.i.r.p. é estatisticamente independente da posição do terminal VSAT.

No Capítulo 4, a modelagem apresentada no no Capitulo 3 á aplicada a cenários de interesse envolvendo a interferência gerada por enlaces inbound de uma rede VSAT/MF-TDMA. Os cenários abordados incluem tanto situações envolvendo regiões geográficas com um único tipo de distribuição de terminais quanto situações envolvendo terminais VSAT distribuídos em uma área geográfica contendo múltiplos tipos de distribuição de terminais. Além dos resultados numéricos obtidos, são apresentadas considerações específicas sobre a identificação de regiões, a atenuação por chuvas, o tamanho das antenas e as densidades de e.i.r.p. utilizadas pelos terminais, além de aspectos relativos à distribuição geográfica de terminais VSAT/MF-TDMA. Para efeitos de validação dos resultados obtidos, os mesmos são comparados com aqueles obtidos a partir de dados reais fornecidos pela operadora de satélites Embratel Star One.

Finalmente, no Capítulo 5, são apresentadas algumas conclusões gerais e sugestões para trabalhos futuros. 


\section{Descrição do Problema}

Em um sistema de comunicações, o sinal emitido por um transmissor sofre degradações devido à influência do ambiente através do qual é transmitido. Dois tipos de degradação são considerados importantes: a degradação produzida por aspectos de propagação e a degradação causada por sinais interferentes $i$ gerados por outros sistemas. Tais fontes de degradação têm um comportamento aleatório e podem ser modeladas probabilísticamente.

Para garantir um desempenho adequado de um enlace, é importante o estabelecimento de critérios de proteção que garantam a operação adequada do enlace, mesmo quando sob condições adversas de propagação e na presença de interferências externas. Estes critérios de proteção são usualmente definidos através da imposição de restrições ao comportamento estatístico da razão portadora-ruído na entrada do receptor do enlace e têm, geralmente, a forma

$$
P\left(\left(\frac{c}{N}\right)<\left(\frac{C}{N}\right)_{j}\right) \leq p_{j} \quad ; j=1, \ldots, J
$$

onde, $c$ representa a potência do sinal recebido, $N$ a potência de ruído térmico na entrada do receptor, $(C / N)_{j}, j=1, \ldots, J$ correspondem a valores pré especificados da razão portadora ruído e $p_{j}$ representam valores máximos das probabilidades com as quais os valores $(C / N)_{j}$ não devem ser excedidos. Note que este tipo de critério de proteção é definido pelo conjunto de pares $\left((C / N)_{j}, p_{j}\right), j=1, \ldots, J$, quando a razão portadora ruído é expressa em dB, o critério de proteção em (2-1) se escreve

$$
P\left(\left(\frac{c}{N}\right)_{\mathrm{dB}}<\left(\frac{C}{N}\right)_{j \mathrm{~dB}}\right) \leq p_{j} \quad ; j=1, \ldots, J
$$

A quantidade $(c / N)$ que aparece em (2-1) e (2-2) representa a razão portadora ruído degradada, ou seja, ela considera os efeitos de propagação e os efeitos de interferências externas $i$. Considerando-se que $x$ e $y$ são variáveis aleatórias que representam, respectivamente, as degradações de razão portadora-ruído devidas à aspectos de propagação e às interferências externas $i$, é possível escrever

$$
\frac{c}{N}=\frac{1}{x} \frac{1}{y}\left(\frac{C}{N}\right)_{C S}
$$

onde $(C / N)_{C S}$ representa a razão portadora-ruído em condições de céu claro 
(clear sky), em dB, (2-3) se escreve

$$
\left(\frac{c}{N}\right)_{\mathrm{dB}}=\left(\frac{C}{N}\right)_{C S_{\mathrm{dB}}}-x_{\mathrm{dB}}-y_{\mathrm{dB}}
$$

onde, $x_{\mathrm{dB}}$ e $y_{\mathrm{dB}}$ são dados respectivamente por $10 \log x$ e $10 \log y$. Alternativamente,

$$
\left(\frac{c}{N}\right)_{\mathrm{dB}}=\left(\frac{C}{N}\right)_{C S_{\mathrm{dB}}}-z_{\mathrm{dB}}
$$

onde,

$$
z_{\mathrm{dB}}=x_{\mathrm{dB}}+y_{\mathrm{dB}} .
$$

Considerando-se (2-5), as restrições em (2-2) se escrevem

$$
P\left(\left(\frac{C}{N}\right)_{C S_{\mathrm{dB}}}-z_{\mathrm{dB}}<\left(\frac{C}{N}\right)_{j \mathrm{~dB}}\right) \leq p_{j} \quad ; j=1, \ldots, J
$$

ou ainda

$$
P\left(z_{\mathrm{dB}}>\left(\frac{C}{N}\right)_{C S_{\mathrm{dB}}}-\left(\frac{C}{N}\right)_{j \mathrm{~dB}}\right) \leq p_{j} \quad ; j=1, \ldots, J
$$

Assim, para verificar se o critério em (2-8) é atendido, é necessaria a determinação do comportamento estatístico da variável aleatória $z_{\mathrm{dB}}$, ou seja, é necessário o conhecimento da função distribuição de probabilidade $p_{z_{\mathrm{dB}}}(Z)$. Considerando-se (2-6), e que as degradações por aspectos de propagação (chuvas) e interferências externas são estatisticamente independentes, tem-se

$$
p_{z_{\mathrm{dB}}}(Z)=p_{x_{\mathrm{dB}}}(Z) * p_{y_{\mathrm{dB}}}(Z) .
$$

Para a determinação da função distribuição de probabilidade $p_{x_{\mathrm{dB}}}(X)$, correspondente à degradação por chuvas, são usualmente considerados os modelos matemáticos estabelecidos no âmbito do Setor de Radiocomunicações da União Internacional de Telecomunicações (ITU-R), como por exemplo o modelo apresentado na Recomendação ITU-R P.837-6 [15].

Note que a degradação $y$ da razão portadora-ruído, devida a interferências externas $i$, se escreve

$$
y=\frac{1}{1+\frac{i}{N}}
$$

uma vez que

$$
\left(\frac{c}{N+i}\right)=\frac{1}{1+\frac{i}{N}}\left(\frac{c}{N}\right) .
$$

A degradação devida a interferências externas, quando expressa em dB, é então 
dada por

$$
y_{\mathrm{dB}}=10 \log \left(\frac{1}{1+\frac{i}{N}}\right)
$$

O relacionamento em (2-12) mostra claramente que o comportamento estatístico da degradação $y_{\mathrm{dB}}$ depende do comportamento estatístico da potência interferente $i$ cuja modelagem é objeto deste trabalho.

Conforme indicado a seguir, no caso de sistemas interferentes MFTDMA, o comportamento estatístico da potência interferente $i$ reflete uma variação temporal $i(t)$ devida às transmissões de diferentes estações terrenas (terminais) multiplexadas no tempo. Com efeito, o segmento terrestre de uma rede VSAT/MF-TDMA é composto por um grande número de terminais (estações terrenas) com antenas de abertura pequena, distribuídas em uma determinada área geográfica, e por uma estação principal de grande porte (Hub station). A comunicação é feita através de dois tipos de enlaces: o primeiro dos terminais $\left(T_{1}^{\prime}, T_{2}^{\prime} \ldots T_{N}^{\prime}\right)$ para a estação principal Hub (inbound), e o segundo da estação $H u b$ para os terminais (outbound), conforme ilustrado nas figuras $2.1(\mathrm{a})$ e $2.1(\mathrm{~b})$.

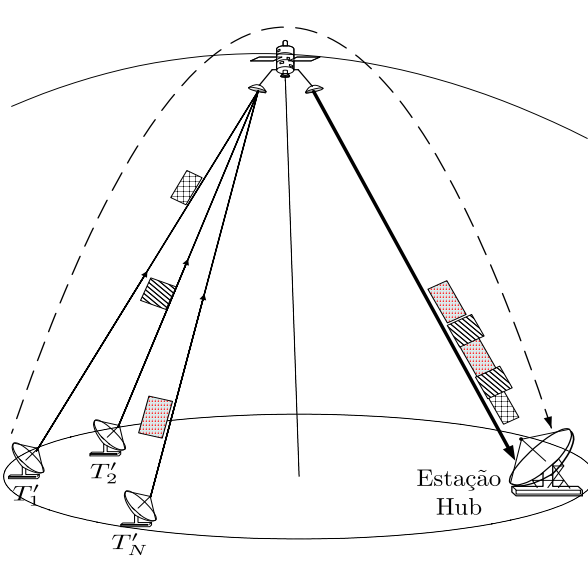

2.1(a): Enlace inbound

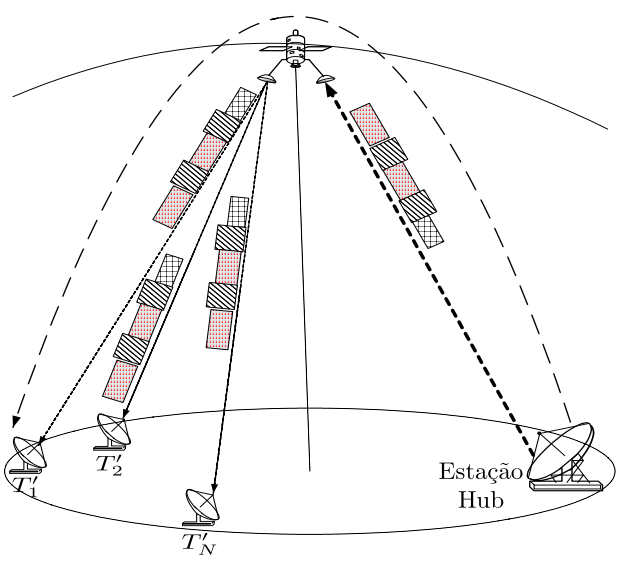

2.1(b): Enlace outbound

Figura 2.1: Direção dos enlaces inbound e outbound, em uma rede VSAT (Very Small Aperture Terminal)

Note que o nível de interferência $i$ introduzido pelos terminais da rede VSAT que usam o protocolo MF-TDMA em seu enlace inbound, varia em função do tempo (time slots), conforme ilustrado na Figura 2.2. Assim, este trabalho tem por objetivo a modelagem probabilística da potência interferente produzida pelo enlace inbound de uma rede VSAT/MF-TDMA no receptor do satélite de uma rede por satélite qualquer, considerada vítima.

A modelagem proposta para a caracterização estatística da potência interferente produzida pelos enlaces inbound de sistemas VSAT/MF-TDMA é apresentada no Capítulo 3. 


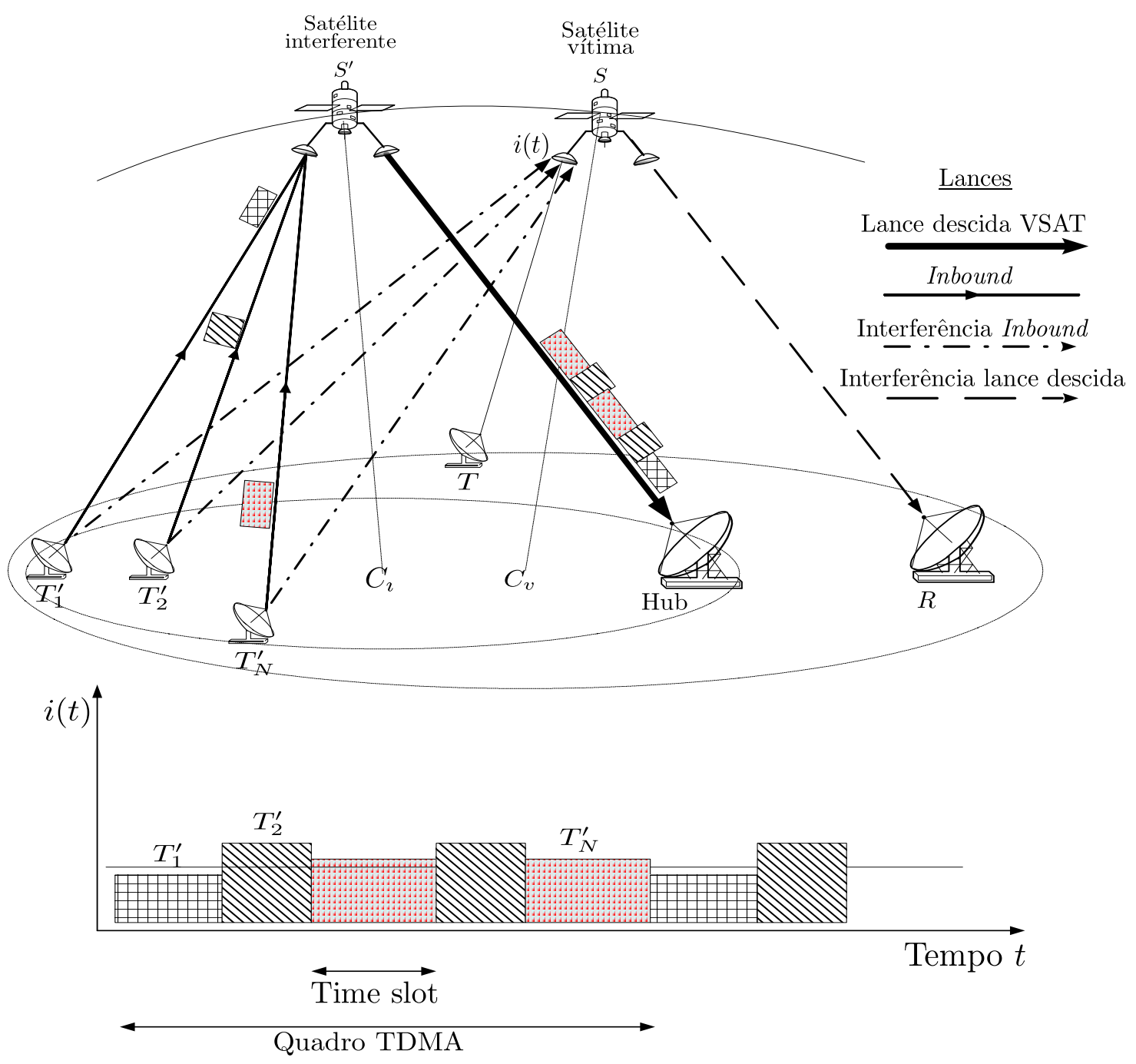

Figura 2.2: Cenário correspondente ao "padrão de interferências" observado no receptor da rede vítima devido à transmissão dos terminais $T_{1}^{\prime}, \ldots, T_{N}^{\prime}$ de uma rede $\mathrm{VSAT/MF-TDMA.}$ 


\section{Modelagem Matemática}

\section{1}

\section{Considerações básicas}

A geometria considerada no cálculo de interferências envolvendo redes VSAT (Very Small Aperture Terminal) que utilizam o protocolo de Acesso Múltiplo por Divisão de Tempo (TDMA), é ilustrada na Figura 3.1

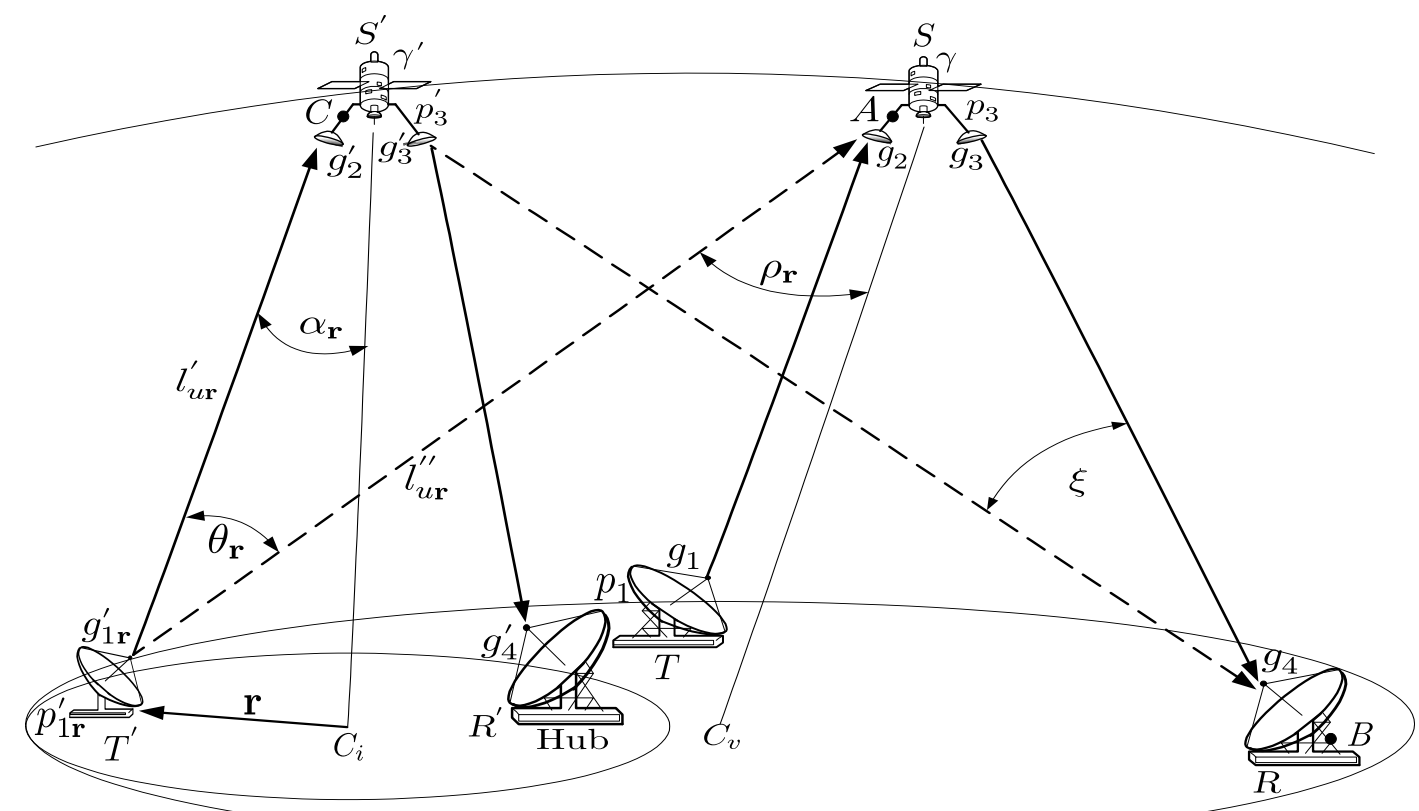

Figura 3.1: Geometria associada ao modelo.

Observa-se na Figura 3.1, duas redes de satélites: uma rede VSAT/MFTDMA interferente que utiliza o satélite $S^{\prime}$ com estação transmissora $T^{\prime}$ localizada numa posição $\mathbf{r}$ dentro da área de cobertura do feixe de recepção do satélite $S^{\prime}$, e uma segunda rede vítima que utiliza o satélite $S$ e na qual considera-se um enlace da estação terrena transmissora $T$ para a estação terrena receptora $R$.

Com base na geometria apresentada na Figura 3.1, a densidade de interferência $i_{u}(\mathbf{r})$ (inbound), produzida na estação terrena vítima $R$ pelo lance de subida do enlace interferente originado em $T^{\prime}$ é dada por

$$
i_{u}(\mathbf{r})=\frac{p_{1 \mathbf{r}}^{\prime} g_{1 \mathbf{r}}^{\prime}\left(\theta_{\mathbf{r}}\right) g_{2}\left(\rho_{\mathbf{r}}\right)}{l_{u \mathbf{r}}^{\prime \prime}} \gamma
$$


onde, $p_{1 \mathbf{r}}^{\prime}$ é a densidade de potência de transmissão do terminal interferente $T^{\prime}$ em $[\mathrm{W} / \mathrm{Hz}], g_{1 \mathbf{r}}^{\prime}\left(\theta_{\mathbf{r}}\right)$ é o ganho da antena transmissora do terminal interferente $T^{\prime}$ na direção do satélite vítima $S, g_{2}\left(\rho_{\mathbf{r}}\right)$ é o ganho da antena receptora do satélite vítima $S$ na direção do terminal interferente $T^{\prime}, l_{u \mathbf{r}}^{\prime \prime}$ é a perda de propagação no espaço livre no percurso entre o terminal $T^{\prime}$ e o satélite vítima $S$ e $\gamma$ é o ganho de transmissão desde a saída da antena receptora do satélite $S$ até a saída da antena receptora da estação terrena vítima $R$ (respectivamente pontos A e B na Figura 3.1). Os índices $\mathbf{r}$ em (3-1) foram adicionados para indicar a dependência das quantidades envolvidas com a posição geográfica da estação terrena transmissora interferente $T^{\prime}$.

A densidade de interferência $i_{d}(\mathbf{r})[\mathrm{W} / \mathrm{Hz}]$, produzida na estação terrena vítima $R$, pelo lance de descida do enlace interferente originado em $T^{\prime}$ é dada por

$$
i_{d}(\mathbf{r})=\frac{p_{1 \mathbf{r}}^{\prime} g_{1 \mathbf{r}}^{\prime}(0) g_{2}^{\prime}\left(\alpha_{\mathbf{r}}\right)}{l_{u \mathbf{r}}^{\prime}} \gamma^{\prime}
$$

onde, $p_{1 \mathbf{r}}^{\prime}$ é a densidade de potência de transmissão em [W/Hz] do terminal interferente $T^{\prime}, g_{1 \mathbf{r}}^{\prime}(0)$ é o ganho da antena transmissora do terminal interferente $T^{\prime}$ na direção do satélite interferente $S^{\prime}, g_{2}^{\prime}\left(\alpha_{\mathbf{r}}\right)$ é o ganho da antena receptora do satélite interferente $S^{\prime}$ na direção do terminal interferente $T^{\prime}, l_{u \mathbf{r}}^{\prime}$ é a perda de propagação no espaço livre no percurso entre o terminal $T^{\prime}$ e o satélite interferente $S^{\prime}$ e $\gamma^{\prime}$ é o ganho de transmissão desde a saída da antena receptora do satélite $S^{\prime}$ até a saída da antena receptora da estação terrena vítima $R$ (respectivamente pontos C e B na Figura 3.1).

Note que (3-1) e (3-2), podem ser expressas em termos da densidade espectral de e.i.r.p. (Effective Isotropic Radiated Power) emitida pelo terminal $T^{\prime}$, definida como

$$
e_{\mathbf{r}}=p_{1 \mathbf{r}}^{\prime} g_{1 \mathbf{r}}^{\prime}(0)
$$

resultando

$$
i_{u}(\mathbf{r})=\frac{e_{\mathbf{r}} g_{1 \mathbf{r}}^{\prime}\left(\theta_{\mathbf{r}}\right) g_{2}\left(\rho_{\mathbf{r}}\right)}{g_{1 \mathbf{r}}^{\prime}(0) l_{u \mathbf{r}}^{\prime \prime}} \gamma
$$

e

$$
i_{d}(\mathbf{r})=\frac{e_{\mathbf{r}} g_{2}^{\prime}\left(\alpha_{\mathbf{r}}\right)}{l_{u \mathbf{r}}^{\prime}} \gamma^{\prime}
$$

A interferência total recebida na estação terrena receptora $R$ do enlace vítima corresponde à soma da interferências devidas aos lances de subida e descida ou seja

$$
i=i_{u}(\mathbf{r})+i_{d}(\mathbf{r})
$$

com $i_{u}(\mathbf{r})$ e $i_{d}(\mathbf{r})$ dados por (3-4) e (3-5) respectivamente. 


\section{2 \\ Determinação da função densidade de probabilidade da densidade de potência interferente $p_{i}(I)$}

Observa-se na Figura 3.1, que a densidade de potência interferente $i$ que atinge a estação terrena $R$ do sistema vítima depende, dentre outros parâmetros, da posição $\mathbf{r}$ da estação terrena transmissora interferente $T^{\prime}$, da densidade de e.i.r.p. $\left(e_{\mathbf{r}}\right)$ na direção de máxima radiação de sua antena transmissora e do angulo off-axis $\theta_{\mathbf{r}}$. Neste trabalho, estas três variáveis $\left(\mathbf{r}, e_{\mathbf{r}}\right.$ e $\theta_{\mathbf{r}}$ ) são modeladas por variáveis aleatórias. A aleatoriedade de $\theta_{\mathbf{r}}$ se justifica com base nos erros de apontamento das antenas transmissoras dos terminais VSAT. Com esta modelagem, a densidade de potência interferente $i$, dada por (3-6), (3-4) e(3-5), será também uma variável aleatória, podendo sua função densidade de probabilidade ser escrita como

$$
p_{i}(I)=\int_{\Omega_{\mathbf{r}}} \int_{-\infty}^{\infty} \int_{-\infty}^{\infty} p_{i \mathbf{r} e_{\mathbf{r}} \theta_{\mathbf{r}}}(I, \mathbf{R}, E, \Theta) d E d \Theta d \mathbf{R}
$$

onde $\Omega_{\mathbf{r}}$ representa o espaço de variação do vetor $\mathbf{r}$, e $p_{i \mathbf{r} e_{\mathbf{r}} \theta_{\mathbf{r}}}(I, \mathbf{R}, E, \Theta)$ é a função densidade de probabilidade conjunta das variáveis $i, \mathbf{r}, e_{\mathbf{r}}$ e $\theta_{\mathbf{r}}$ que, por sua vez, pode ser escrita como

$$
p_{i \mathbf{r} e_{\mathbf{r}} \theta_{\mathbf{r}}}(I, \mathbf{R}, E, \Theta)=p_{i \mid \mathbf{r}=\mathbf{R}, e_{\mathbf{r}}=E, \theta_{\mathbf{r}}=\Theta}(I) p_{\mathbf{r} e_{\mathbf{r}} \theta_{\mathbf{r}}}(\mathbf{R}, E, \Theta)
$$

com $p_{\mathbf{r} e_{\mathbf{r}} \theta_{\mathbf{r}}}(\mathbf{R}, E, \Theta)$ denotando a função de densidade de probabilidade conjunta de $\mathbf{r}, e_{\mathbf{r}}$ e $\theta_{\mathbf{r}}$, que pode ser escrita como

$$
p_{\mathbf{r} e_{\mathbf{r}} \theta_{\mathbf{r}}}(\mathbf{R}, E, \Theta)=p_{e_{\mathbf{r}} \theta_{\mathbf{r}} \mid \mathbf{r}=\mathbf{R}}(E, \Theta) p_{\mathbf{r}}(\mathbf{R}) .
$$

Considerando-se que, para uma determinada posição $\mathbf{r}$, a densidade e.i.r.p. $e_{\mathbf{r}}$ e o ângulo off-axis $\theta_{\mathbf{r}}$, são estatisticamente independentes, tem-se

$$
p_{e_{\mathbf{r}} \theta_{\mathbf{r}} \mid \mathbf{r}=\mathbf{R}}(E, \Theta)=p_{e_{\mathbf{r}} \mid \mathbf{r}=\mathbf{R}}(E) p_{\theta_{\mathbf{r}} \mid \mathbf{r}=\mathbf{R}}(\Theta)
$$

Substituindo (3-10), (3-9), (3-8) em (3-7), obtém-se

$$
p_{i}(I)=\int_{\Omega_{\mathbf{r}}} \int_{-\infty}^{\infty} \int_{-\infty}^{\infty} p_{i \mid \mathbf{r}=\mathbf{R}, e_{\mathbf{r}}=E, \theta_{\mathbf{r}}=\Theta}(I) p_{e_{\mathbf{r}} \mid \mathbf{r}=\mathbf{R}}(E) p_{\theta_{\mathbf{r}} \mid \mathbf{r}=\mathbf{R}}(\Theta) p_{\mathbf{r}}(\mathbf{R}) d E d \Theta d \mathbf{R}
$$

Observe que, dado $e_{\mathbf{r}}=E, \mathbf{r}=\mathbf{R}$ e $\theta_{\mathbf{r}}=\Theta_{\mathbf{R}}$, a variável aleatória $i$ assume um valor fixo conhecido, $\mathcal{I}(E, \mathbf{R}, \Theta)$, com probabilidade um. Neste caso a primeira função densidade de probabilidade condicional no integrando 
de (3-11), se escreve

$$
p_{i \mid \mathbf{r}=\mathbf{R}, e_{\mathbf{r}}=E, \theta_{\mathbf{r}}=\Theta}(I)=\delta(I-\mathcal{I}(E, \mathbf{R}, \Theta))
$$

$\operatorname{com} \delta($ ) denotando a função impulso (Dirac delta function). Assim, a função densidade de probabilidade em (3-11) escreve

$$
p_{i}(I)=\int_{\Omega_{\mathbf{r}}} p_{\mathbf{r}}(\mathbf{R}) \int_{-\infty}^{\infty} p_{\theta_{\mathbf{r}} \mid \mathbf{r}=\mathbf{R}}(\Theta) \int_{-\infty}^{\infty} \delta(I-\mathcal{I}(E, \mathbf{R}, \Theta)) p_{e_{\mathbf{r}} \mid \mathbf{r}=\mathbf{R}}(E) d E d \Theta d \mathbf{R}
$$

Neste ponto é importante ressaltar que usualmente a e.i.r.p. emitida por um terminal VSAT está diretamente ligada ao tamanho (ganho) de sua antena transmissora. Assim, é razoável considerar que, dada a posição $\mathbf{r}=\mathbf{R}$ do terminal VSAT, a densidade de e.i.r.p. (W/Hz) por ele transmitida seja modelada por uma variável aleatória discreta que assume valores num conjunto discreto e finito $\left\{E_{j_{\mathbf{R}}}, j=1, \ldots, N_{\mathbf{R}}\right\}$, onde cada um dos valores possíveis está relacionado a um determinado tamanho de antena e à posição geográfica $\mathbf{R}$ do terminal VSAT interferente $T^{\prime}$. Tem-se assim

$$
p_{e_{\mathbf{r}} \mid \mathbf{r}=\mathbf{R}}(E)=\sum_{j=1}^{N_{\mathbf{R}}} P_{j_{\mathbf{R}}} \delta\left(E-E_{j_{\mathbf{R}}}\right)
$$

onde

$$
P_{j_{\mathbf{R}}}=P\left(e_{\mathbf{r}}=E_{j_{\mathbf{R}}} \mid \mathbf{r}=\mathbf{R}\right) .
$$

Neste caso, a integral mais interna em (3-13) se escreve

$$
\int_{-\infty}^{\infty} \delta(I-\mathcal{I}(E, \mathbf{R}, \Theta)) p_{e_{\mathbf{r}} \mid \mathbf{r}=\mathbf{R}}(E) d E=\sum_{j=1}^{N_{\mathbf{R}}} P_{j_{\mathbf{R}}} \delta\left(I-\mathcal{I}\left(E_{j_{\mathbf{R}}}, \mathbf{R}, \Theta\right)\right)
$$

e, consequentemente,

$$
p_{i}(I)=\int_{\Omega_{\mathbf{r}}} p_{\mathbf{r}}(\mathbf{R}) \sum_{j=1}^{N_{\mathbf{R}}} P_{j_{\mathbf{R}}} \int_{-\infty}^{\infty} p_{\theta_{\mathbf{r}} \mid \mathbf{r}=\mathbf{R}}(\Theta) \delta\left(I-\mathcal{I}\left(E_{j_{\mathbf{R}}}, \mathbf{R}, \Theta\right)\right) . d \Theta d \mathbf{R}
$$

A função distribuição de probabilidade da densidade de potência interferente, definida como $P(i \leq I)$, é obtida integrando (3-17), resultando

$$
F_{i}(I)=\int_{\Omega_{\mathbf{r}}} p_{\mathbf{r}}(\mathbf{R}) \sum_{j=1}^{N_{\mathbf{R}}} P_{j_{\mathbf{R}}} \int_{-\infty}^{\infty} p_{\theta_{\mathbf{r}} \mid \mathbf{r}=\mathbf{R}}(\Theta) u\left(I-\mathcal{I}\left(E_{j_{\mathbf{R}}}, \mathbf{R}, \Theta\right)\right) d \Theta d \mathbf{R}
$$

onde $u($ ) denota a função degrau unitário. Note que (3-18) pode ainda ser 
escrita como

$$
F_{i}(I)=\int_{\Omega_{\mathbf{r}}} p_{\mathbf{r}}(\mathbf{R}) \sum_{j=1}^{N_{\mathbf{R}}} P_{j_{\mathbf{R}}} H_{\mathbf{R} j}(I) d \mathbf{R}
$$

onde

$$
H_{\mathbf{R} j}(I)=\int_{-\infty}^{\infty} p_{\theta_{\mathbf{r}} \mid \mathbf{r}=\mathbf{R}}(\Theta) u\left(I-\mathcal{I}\left(E_{j_{\mathbf{R}}}, \mathbf{R}, \Theta\right)\right) d \Theta .
$$

Note que (3-19), pode ainda ser escrita como

$$
F_{i}(I)=E[g(\mathbf{r})]
$$

onde

$$
g(\mathbf{r})=\sum_{j=1}^{N_{\mathbf{R}}} P_{j_{\mathbf{r}}} H_{\mathbf{r} j}(I)
$$

\section{3}

\section{Expressão Analítica da Função $H_{\mathbf{R} j}(I)$}

Para obter uma expressão analítica para a função $H_{\mathbf{R} j}(I)$ note, em primeiro lugar, que considerando-se (3-4) (3-5) e (3-6), o valor $\mathcal{I}\left(E_{j_{\mathbf{R}}}, \mathbf{R}, \Theta\right)$ pode ser escrito como

$$
\mathcal{I}\left(E_{j_{\mathbf{R}}}, \mathbf{R}, \Theta\right)=K_{1 \mathbf{R} j} g_{1 \mathbf{R} j}^{\prime}(\Theta)+K_{2 \mathbf{R} j}
$$

onde

$$
K_{1 \mathbf{R} j}=\frac{E_{j_{\mathbf{R}}} g_{2}\left(\rho_{\mathbf{R}}\right)}{g_{1 \mathbf{R} j}^{\prime}(0) l_{u_{\mathbf{R}}}^{\prime \prime}} \gamma
$$

e

$$
K_{2 \mathbf{R} j}=\frac{E_{j_{\mathbf{R}}} g_{2}^{\prime}\left(\alpha_{\mathbf{R}}\right)}{l_{u_{\mathbf{R}}}^{\prime}} \gamma^{\prime}
$$

Em (3-23) e (3-24) $g_{1 \mathbf{R} j}^{\prime}($ ) representa o diagrama de radiação da antena transmissora do terminal interferente $T^{\prime}$, que está associado ao nível $E_{j_{\mathbf{R}}}$ de densidade de e.i.r.p. de um terminal localizado em $\mathbf{r}=\mathbf{R}$. Este diagrama de radiação tem, usualmente, a forma

$$
g_{1 \mathbf{R} j}^{\prime}(\Theta)= \begin{cases}f_{1 \mathbf{R} j}(\Theta) & ; 0 \leq \Theta<\Theta_{1 \mathbf{R} j} \\ G_{1 \mathbf{R} j} & ; \quad \Theta_{1 \mathbf{R} j} \leq \Theta<\Theta_{2 \mathbf{R} j} \quad ; \quad j=1, \ldots, N_{\mathbf{R}} \\ f_{2 \mathbf{R} j}(\Theta) & ; \quad \Theta_{2 \mathbf{R} j} \leq \Theta<\Theta_{3 \mathbf{R} j} \\ G_{2 \mathbf{R} j} & ; \quad \Theta_{3 \mathbf{R} j} \leq \Theta\end{cases}
$$


onde as funções $f_{1 \mathbf{R} j}(\Theta)$ e $f_{2 \mathbf{R} j}(\Theta)$ descrevem, respectivamente, o lóbulo principal (main lobes) e os lóbulos laterais (side-lobe) da antena e $G_{1 \mathbf{R} j}$ e $G_{2 \mathbf{R} j}$ correspondem, respectivamente, aos níveis de lóbulos laterais próximos (near side-lobe level) e distantes (far side-lobe level). Observe que o ganho máximo $G_{0 \mathbf{R} j}$ da antena é igual a $f_{1 \mathbf{R} j}(0)$. Esta forma de diagrama de radiação em dB é ilustrada na Figura 3.2.

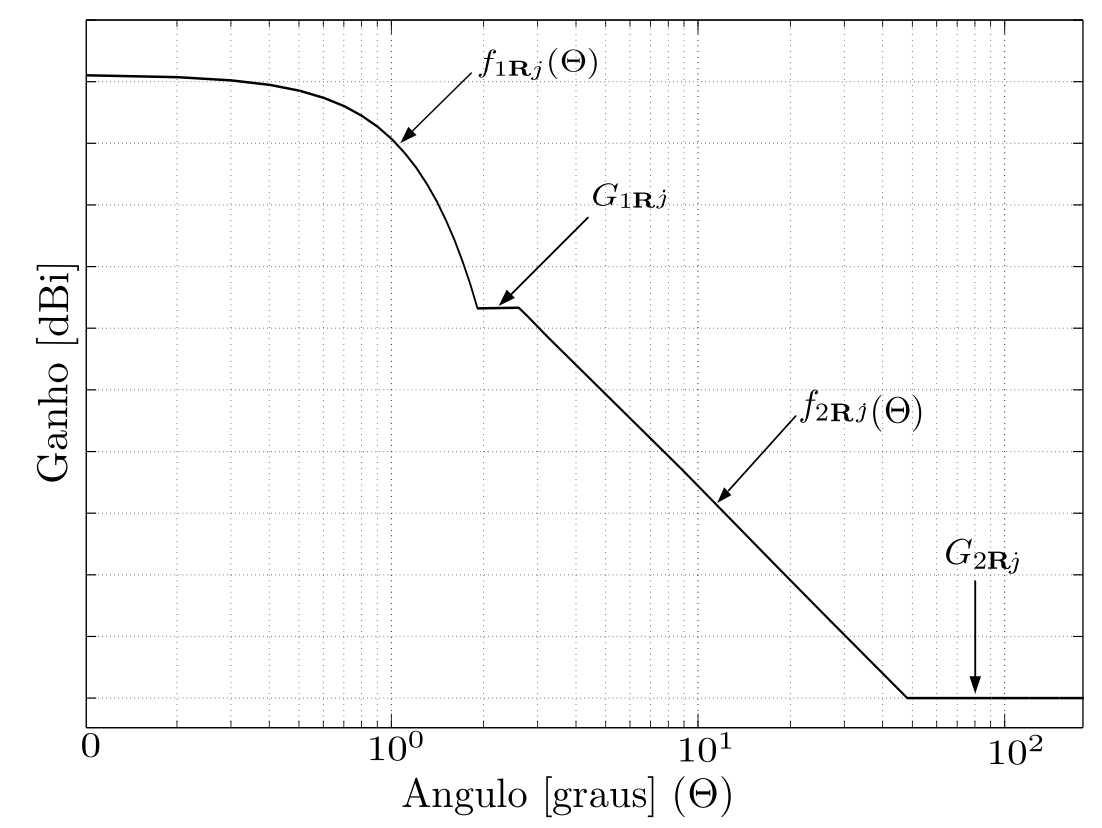

Figura 3.2: Diagrama de radiação usual da antena do terminal interferente $T^{\prime}$.

Considerando (3-20) e (3-23), é possível verificar que $H_{\mathbf{R} j}(I)$ pode ser escrito como

$$
H_{\mathbf{R} j}(I)=\int_{\mathcal{S}_{\mathbf{R} j}} p_{\theta_{\mathbf{r}} \mid \mathbf{r}=\mathbf{R}}(\Theta) d \Theta
$$

com

$$
\mathcal{S}_{\mathbf{R} j}=\left\{\Theta \in \Omega_{\theta} \mid g_{1 \mathbf{R} j}^{\prime}(\Theta) \leq \frac{I-K_{2 \mathbf{R} j}}{K_{1 \mathbf{R} j}}\right\}
$$

Como em [4], os erros de apontamento, em azimute $\left(\phi_{a}\right)$ e elevação $\left(\phi_{e}\right)$, das antenas transmissoras dos terminais VSAT são modeladas por variáveis aleatórias gaussianas, estatisticamente independentes, com médias nulas e variâncias $\sigma^{2}$. Na Figura 3.3, é apresentada a geometria associada aos erros de apontamento. Consideranda dita geometria, pode ser facilmente deduzida a expressão para $\theta_{\mathbf{r}}$

$$
\theta_{\mathbf{r}} \approx \sqrt{\left(\phi_{a}-\phi_{a_{\mathbf{R}}}\right)^{2}+\left(\phi_{e}-\phi_{e_{\mathbf{R}}}\right)^{2}}
$$

onde $\phi_{a_{\mathbf{R}}}$ e $\phi_{e_{\mathbf{R}}}$ denotam, respectivamente os ângulos de azimute e elevação da antena transmissora correspondente ao terminal interferente $T^{\prime}$ quando 
está apontando na direção do satélite vitima $S$. Em (3-29), $\phi_{a}$ e $\phi_{e}$ são variáveis aleatórias gaussianas estatisticamente independentes com media nula e variância $\sigma^{2}$. Dado que $\mathbf{r}=\mathbf{R}$, é possível mostrar que $\theta_{\mathbf{r}}$, tem uma função densidade de probabilidade Rice [7], [8], dada por,

$$
p_{\theta_{\mathbf{r}} \mid \mathbf{r}=\mathbf{R}}(\Theta)=\frac{\Theta}{\sigma^{2}} \mathrm{e}\left(-\frac{\Theta^{2}+A_{\mathbf{R}}^{2}}{2 \sigma^{2}}\right) I_{0}\left(\frac{\Theta A_{\mathbf{R}}}{\sigma^{2}}\right) u(\Theta)
$$

onde

$$
A_{\mathbf{R}}=\sqrt{\phi_{a_{\mathbf{R}}}^{2}+\phi_{e_{\mathbf{R}}}^{2}}
$$

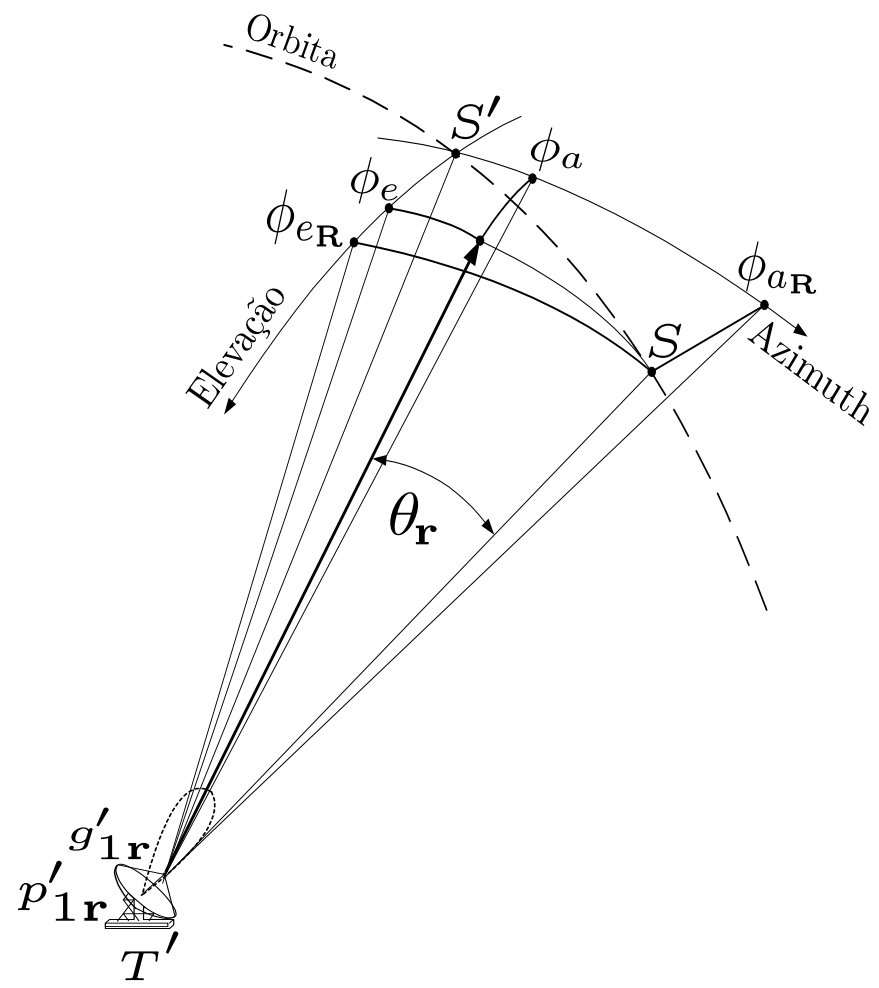

Figura 3.3: Dependência de $\theta_{\mathbf{r}}$ sobre os erros de apontamento da antena em azimute e elevação.

É interessante ressaltar que, em [4], o efeito dos erros de apontamento das antenas dos terminais VSAT sobre o ângulo $\theta_{\mathbf{r}}$ e, consequentemente, sobre a função $H_{\mathbf{R} j}(I)$ definida em (3-27) e (3-28), foi considerado pelo método de Monte Carlo. Neste trabalho, o resultado em (3-30) é utilizado em (3-27) na determinação de uma expressão analítica para $H_{\mathbf{R} j}(I)$. Além disso, o diagrama de radiação em (3-26) foi utilizado para determinar a região de integração $\mathcal{S}_{\mathbf{R} j}$ 
definida em (3-28). Após algumas manipulações algébricas é possível obter

$$
H_{\mathbf{R} j}(I)= \begin{cases}0 \quad ; I<G_{2 \mathbf{R} j} K_{1 \mathbf{R} j}+K_{2 \mathbf{R} j} \\ Q_{1}\left(\frac{A_{\mathbf{R}}}{\sigma}, \frac{f_{2 \mathbf{R} j}^{-1}\left(\frac{I-K_{2 \mathbf{R} j}}{K_{1 \mathbf{R} j}}\right)}{\sigma}\right) ; & G_{2 \mathbf{R} j} K_{1 \mathbf{R} j}+K_{2 \mathbf{R} j} \leq I<G_{1 \mathbf{R} j} K_{1 \mathbf{R} j}+K_{2 \mathbf{R} j} \\ Q_{1}\left(\frac{A_{\mathbf{R}}}{\sigma}, \frac{f_{1 \mathbf{R} j}^{-1}\left(\frac{I-K_{2 \mathbf{R} j}}{K_{1 \mathbf{R} j}}\right)}{\sigma}\right) ; & G_{1 \mathbf{R} j} K_{1 \mathbf{R} j}+K_{2 \mathbf{R} j} \leq I<G_{0 \mathbf{R} j} K_{1 \mathbf{R} j}+K_{2 \mathbf{R} j} \\ 1 & ; G_{0 \mathbf{R} j} K_{1 \mathbf{R} j}+K_{2 \mathbf{R} j} \leq I\end{cases}
$$

onde $Q_{1}(\cdot, \cdot)$, denota a função Marcum- $Q$ generalizada, $Q_{1}$ definida por

$$
Q_{1}(a, b)=\int_{b}^{\infty} x e^{-\frac{x^{2}+a^{2}}{2}} I_{0}(a x) d x
$$

Finalmente, a função distribuição de probabilidade da potência interferente é determinada utilizando-se (3-19) e (3-32). A modelagem probabilística apresentada nesta seção foi apresentada em artigo publicado nos anais da CCIS 2016 [16].

\subsection{1}

\section{Caso particular: densidade de e.i.r.p. estatisticamente independente da posição do terminal VSAT}

No caso em que a densidade de e.i.r.p. e $_{\mathbf{r}}$ é estatisticamente independente da posição $\mathbf{r}$ dos terminais VSAT tem-se, de (3-14)

$$
p_{e_{\mathbf{r}} \mid \mathbf{r}=\mathbf{R}}(E)=p_{e_{\mathbf{r}}}(E)=\sum_{j=1}^{N} P_{j} \delta\left(E-E_{j}\right) \quad \forall \mathbf{r} \in \Omega_{\mathbf{r}}
$$

com

$$
P_{j}=P\left(e_{\mathbf{r}}=E_{j}\right)
$$

ou seja, a variável aleatória $e_{\mathbf{r}}$ que caracteriza a densidade de e.i.r.p., toma valores num mesmo conjunto discreto, $\left\{E_{j}, j=1, \ldots, N\right\}$, qualquer que seja a posição $\mathbf{r}$ do terminal VSAT interferente.

Neste caso, (3-17) e (3-18) tornam-se, respectivamente,

$$
p_{i}(I)=\sum_{j=1}^{N} P_{j} \int_{\Omega_{\mathbf{r}}} p_{\mathbf{r}}(\mathbf{R}) \int_{-\infty}^{\infty} p_{\theta_{\mathbf{r}} \mid \mathbf{r}=\mathbf{R}}(\Theta) \delta\left(I-\mathcal{I}\left(E_{j}, \mathbf{R}, \Theta\right)\right) d \Theta d \mathbf{R}
$$


e

$$
F_{i}(I)=\sum_{j=1}^{N} P_{j} \int_{\Omega_{\mathbf{r}}} p_{\mathbf{r}}(\mathbf{R}) \int_{-\infty}^{\infty} p_{\theta_{\mathbf{r}} \mid \mathbf{r}=\mathbf{R}}(\Theta) u\left(I-\mathcal{I}\left(E_{j}, \mathbf{R}, \Theta\right)\right) d \Theta d \mathbf{R}
$$

Consequentemente, (3-19) e (3-20) são re-escritas como

$$
F_{i}(I)=\sum_{j=1}^{N} P_{j} \int_{\Omega_{\mathbf{r}}} p_{\mathbf{r}}(\mathbf{R}) H_{\mathbf{R} j}(I) d \mathbf{R}
$$

onde

$$
H_{\mathbf{R} j}(I)=\int_{-\infty}^{\infty} p_{\theta_{\mathbf{r}} \mid \mathbf{r}=\mathbf{R}}(\Theta) u\left(I-\mathcal{I}\left(E_{j}, \mathbf{R}, \Theta\right)\right) d \Theta .
$$

Observe ainda que, neste caso particular, os diagramas de radiação $\left\{g_{1 \mathbf{R} j}^{\prime}(\Theta), j=1, \ldots, N_{\mathbf{R}}\right\}$ das antenas transmissoras dos terminais VSAT, dados por (3-26), não dependem de $\mathbf{R}$, podendo ser escritos na forma

$$
g_{1 j}^{\prime}(\Theta)= \begin{cases}f_{1 j}(\Theta) & ; \quad 0 \leq \Theta<\Theta_{1 j} \\ G_{1 j} & ; \quad \Theta_{1 j} \leq \Theta<\Theta_{2 j} \quad ; \quad j=1, \ldots, N \\ f_{2 j}(\Theta) & ; \quad \Theta_{2 j} \leq \Theta<\Theta_{3 j} \\ G_{2 j} & ; \quad \Theta_{3 j} \leq \Theta\end{cases}
$$

sendo o ganho máximo $G_{0 j}$ dado por $f_{1 j}(0)$.

Finalmente, a expressão analítica da função $H_{\mathbf{R} j}(I)$ é, neste caso, escrita como

$$
H_{\mathbf{R} j}(I)= \begin{cases}0 & ; I<G_{2 j} K_{1 \mathbf{R} j}+K_{2 \mathbf{R} j} \\ Q_{1}\left(\frac{A_{\mathbf{R}}}{\sigma}, \frac{f_{2 j}^{-1}\left(\frac{I-K_{2 \mathbf{R} j}}{K_{1 \mathbf{R} j}}\right)}{\sigma}\right) ; & G_{2 j} K_{1 \mathbf{R} j}+K_{2 \mathbf{R} j} \leq I<G_{1 j} K_{1 \mathbf{R} j}+K_{2 \mathbf{R} j} \\ Q_{1}\left(\frac{A_{\mathbf{R}}}{\sigma}, \frac{f_{1 j}^{-1}\left(\frac{I-K_{2 \mathbf{R} j}}{K_{1 \mathbf{R} j}}\right)}{\sigma}\right) & ; G_{1 j} K_{1 \mathbf{R} j}+K_{2 \mathbf{R} j} \leq I<G_{0 j} K_{1 \mathbf{R} j}+K_{2 \mathbf{R} j} \\ 1 \quad & ; G_{0 j} K_{1 \mathbf{R} j}+K_{2 \mathbf{R} j} \leq I\end{cases}
$$

onde $A_{\mathbf{R}}$ é dado por (3-31), $Q_{1}(\cdot, \cdot)$ é a função Marcum- $Q$ generalizada, definida em (3-33), sendo $K_{1 \mathbf{R} j}$ e $K_{2 \mathbf{R} j}$ dados por

$$
K_{1 \mathbf{R} j}=\frac{E_{j} g_{2}\left(\rho_{\mathbf{R}}\right)}{g_{1 j}^{\prime}(0) l_{u_{\mathbf{R}}}^{\prime \prime}} \gamma
$$

$\mathrm{e}$

$$
K_{2 \mathbf{R} j}=\frac{E_{j} g_{2}^{\prime}\left(\alpha_{\mathbf{R}}\right)}{l_{u_{\mathbf{R}}}^{\prime}} \gamma^{\prime}
$$




\section{4}

\section{Consideração de regiões contendo diferentes características de distri- buição de terminais}

O teorema da probabilidade total permite escrever a função densidade de probabilidade da posição geográfica dos terminais VSAT em função das funções densidade de probabilidade condicionais que modelam a distribuição de terminais VSAT em regiões geográficas $\left\{\mathcal{R}_{1}, \ldots, \mathcal{R}_{m}\right\}$ com caraterísticas específicas, ou seja,

$$
p_{\mathbf{r}}(\mathbf{R})=\sum_{m=1}^{M} P\left(\mathbf{r} \in \mathcal{R}_{m}\right) p_{\mathbf{r} \mid \mathbf{r} \in \mathcal{R}_{m}}(\mathbf{R})
$$

Note que as funções densidade de probabilidade condicionais em (3-44) são dadas por

$$
p_{\mathbf{r} \mid \mathbf{r} \in \mathcal{R}_{m}}(\mathbf{R})=\left\{\begin{array}{cl}
\frac{1}{P\left(\mathbf{r} \in \mathcal{R}_{m}\right)} p_{\mathbf{r}}(\mathbf{R}) & ; \quad \mathbf{R} \in \mathcal{R}_{m} \\
0 & ; \quad \mathbf{R} \notin \mathcal{R}_{m}
\end{array}\right.
$$

Estas funções densidade de probabilidade podem, por exemplo, ser associadas a processos estocásticos pontuais bidimensionais $\left(\mathbf{r} \in \mathbb{R}^{2}\right)$ escolhidos, de modo a modelar adequadamente a distribuição de terminais VSAT na região considerada $\mathcal{R}_{m}$.

Neste caso, (3-19) se escreve

$$
F_{i}(I)=\sum_{m=1}^{M} P\left(\mathbf{r} \in \mathcal{R}_{m}\right) \int_{\mathcal{R}_{m}} p_{\mathbf{r} \mid \mathbf{r} \in \mathcal{R}_{m}}(\mathbf{R}) \sum_{j=1}^{N_{\mathbf{R}}} P_{j_{\mathbf{R}}} H_{\mathbf{R} j}(I) d \mathbf{R}
$$

com $H_{\mathbf{R} j}(I)$ dado por (3-32).

\subsection{1}

Caso particular: densidade de e.i.r.p. estatisticamente independente da posição do terminal VSAT, em cada região $\mathcal{R}_{m}$

Quando a densidade de e.i.r.p. $e_{\mathbf{r}}$ é estatisticamente independente da posição $\mathbf{r}$ dos terminais VSAT em uma dada região, tem-se

$$
p_{e_{\mathbf{r}} \mid \mathbf{r} \in \mathcal{R}_{m}}(E)=\sum_{j=1}^{N m} P_{j m} \delta\left(E-E_{j m}\right) \quad \forall \mathbf{r} \in \mathcal{R}_{m}, m=1, \ldots, N_{m}
$$

com

$$
P_{j m}=P\left(e_{\mathbf{r}}=E_{j m} \mid \mathbf{r} \in \mathcal{R}_{m}\right)
$$


ou seja, em cada região $\mathcal{R}_{m}$, a variável aleatória $e_{\mathbf{r}}$ que caracteriza a densidade de e.i.r.p., toma valores num mesmo conjunto discreto, $\left\{E_{j m}, j=1, \ldots, N_{m}\right\}$, qualquer que seja a posição $\mathbf{r}$ do terminal VSAT interferente na região $\mathcal{R}_{m}$.

Observe que, neste caso particular, os possíveis diagramas de radiação em (3-26) são os mesmos para terminais localizados em uma mesma região $\Omega_{m}$, ou seja,

$$
g_{1 j m}^{\prime}(\Theta)= \begin{cases}f_{1 j m}(\Theta) & ; \quad 0 \leq \Theta<\Theta_{1 j m} \\ G_{1 j m} & ; \Theta_{1 j m} \leq \Theta<\Theta_{2 j m} \quad ; \quad j=1, \ldots, N_{m} . \\ f_{2 j m}(\Theta) & ; \quad \Theta_{2 j m} \leq \Theta<\Theta_{3 j m} \\ G_{2 j m} & ; \quad \Theta_{3 j m} \leq \Theta\end{cases}
$$

sendo o ganho máximo $G_{0 j m}$ dado por $f_{1 j m}(0)$.

Além disso, em diferentes regiões $\mathcal{R}_{m}$, os parâmetros $K_{1 \mathbf{R} j}$ e $K_{2 \mathbf{R} j}$, definidos em (3-24) e (3-25), correspondem a diferentes funções de $\mathbf{R}$ sendo, neste caso escritos como

$$
K_{1 \mathbf{R} j m}=\frac{E_{j m} g_{2}\left(\rho_{\mathbf{R}}\right)}{g_{1 j m}^{\prime}(0) l_{u_{\mathbf{R}}}^{\prime \prime}} \gamma
$$

e

$$
K_{2 \mathbf{R} j m}=\frac{E_{j m} g_{2}^{\prime}\left(\alpha_{\mathbf{R}}\right)}{l_{u_{\mathbf{R}}}^{\prime}} \gamma^{\prime} .
$$

Observe, a partir de (3-49) a (3-51), que a função $H_{\mathbf{R} j}(I)$ definida em (3-32) irá também depender da região $\mathcal{R}_{m}$ na qual o terminal VSAT interferente se encontra. Tem-se assim

$$
H_{\mathbf{R} j m}(I)= \begin{cases}0 & ; I<G_{2 j m} K_{1 \mathbf{R} j m}+K_{2 \mathbf{R} j m} \\ Q_{1}\left(\frac{A_{\mathbf{R}}}{\sigma}, \frac{f_{2 j m}^{-1}\left(\frac{I-K_{2 \mathbf{R} j m}}{K_{1 \mathbf{R} j m}}\right)}{\sigma}\right) ; & G_{2 j m} K_{1 \mathbf{R} j m}+K_{2 \mathbf{R} j m} \leq I<G_{1 j m} K_{1 \mathbf{R} j m}+K_{2 \mathbf{R} j m} \\ Q_{1}\left(\frac{A_{\mathbf{R}}}{\sigma}, \frac{f_{1 j m}^{-1}\left(\frac{I-K_{2 \mathbf{R} j m}}{K_{1 \mathbf{R} j m}}\right)}{\sigma}\right) & ; G_{1 m j} K_{1 \mathbf{R} j m}+K_{2 \mathbf{R} j m} \leq I<G_{0 m j} K_{1 \mathbf{R} j m}+K_{2 \mathbf{R} j m} \\ 1 \quad & ; G_{0 m j} K_{1 \mathbf{R} j m}+K_{2 \mathbf{R} j m} \leq I\end{cases}
$$

onde $A_{\mathbf{R}}$ é dado por $(3-31), Q_{1}(\cdot, \cdot)$ é a função $M a r c u m-Q$ generalizada, sendo $K_{1 \mathbf{R} j m}$ e $K_{2 \mathbf{R} j m}$ dados por (3-50) e (3-51), respectivamente.

Finalmente, considerando-se (3-46), a função distribuição de probabilidade da densidade de potência interferente, se escreve, neste caso particular,

$$
F_{i}(I)=\sum_{m=1}^{M} P\left(\mathbf{r} \in \mathcal{R}_{m}\right) \sum_{j=1}^{N_{m}} P_{j m} \int_{\mathcal{R}_{m}} p_{\mathbf{r} \mid \mathbf{r} \in \mathcal{R}_{m}}(\mathbf{R}) H_{\mathbf{R} j m}(I) d \mathbf{R}
$$


ou ainda,

$$
F_{i}(I)=\sum_{m=1}^{M} P\left(\mathbf{r} \in \mathcal{R}_{m}\right) F_{i_{n} \mid \mathbf{r} \in \mathcal{R}_{m}}(I)
$$

onde

$$
F_{i \mid \mathbf{r} \in \mathcal{R}_{m}}(I)=\sum_{j=1}^{N_{m}} P_{j m} \int_{\mathcal{R}_{m}} p_{\mathbf{r} \mid \mathbf{r} \in \mathcal{R}_{m}}(\mathbf{R}) H_{\mathbf{R} j m}(I) d \mathbf{R}
$$

Note que (3-55) pode ainda ser escrita como

$$
F_{i \mid \mathbf{r} \in \mathcal{R}_{m}}(I)=\sum_{j=1}^{N_{m}} P_{j m} E\left[H_{\mathbf{r} j m}(I) \mid \mathbf{r} \in \mathcal{R}_{m}\right]
$$

Conforme mencionado no inicio desta seção, as funções densidade de probabilidade condicionais $p_{\mathbf{r} \mid \mathbf{r} \in \mathcal{R}_{m}}(\mathbf{R})$, podem ser associadas a processos estocásticos pontuais bidimensionais $\left(\mathbf{r} \in \mathbb{R}^{2}\right)$ convenientemente escolhidos. A caracterização estocástica deste tipo de processos é objeto do estudo de uma área de pesquisa matemática conhecida como Geometria Estocástica [9]. Os processos pontuais utilizados neste trabalho para a caracterização da distribuição dos terminais transmissores VSAT, incluem os modelos de processos Binomial e Poisson. Uma descrição sucinta de cada um destes modelos é apresentada no Apêndice A. 


\section{Resultados Numéricos}

Para ilustrar a aplicabilidade do modelo desenvolvido no Capítulo 3 são apresentados neste capítulo, resultados numéricos correspondentes a exemplos específicos dentro de um cenário envolvendo a interferência produzida pelos enlaces (inbound) de uma rede VSAT/TDMA com terminais distribuídos em uma determinada área geográfica sobre um enlace de uma rede que utiliza um satélite adjacente (enlace vítima). Os resultados obtidos com o modelo proposto são comparados aos obtidos a partir de dados reais fornecidos pela operadora de satélites Embratel Star One.

Para a apresentação dos resultados, a densidade de potência interferente $i$ foi normalizada relativamente a um valor de referência $i_{r e f}$, ou seja, considerouse a densidade de potência interferente normalizada dada por

$$
i_{n}=\frac{i}{i_{r e f}}
$$

sendo o valor de referência $i_{\text {ref }}$ definido por

$$
i_{r e f}=\max _{j} i_{r e f_{j}}
$$

com $i_{r e f_{j}}$ sendo o valor da densidade de potência interferente afetando o enlace vítima quando um terminal interferente VSAT, utilizando uma antena transmissora de diâmetro $d_{j}$, é colocado na posição geográfica da área de serviço do sistema VSAT que corresponde ao maior ganho da antena receptora do satélite vítima, e os erros de apontamento em azimute e elevação $\left(\phi_{a}\right.$ e $\left.\phi_{e}\right)$ são iguais a zero.

Os resultados obtidos são apresentados em termos da função distribuição de probabilidade complementar da variável

$$
i_{n_{\mathrm{dB}}}=10 \log i_{n}
$$

ou seja, em termos de $1-F_{i_{n_{\mathrm{dB}}}}(I)$. 


\section{1}

\section{Considerações específicas sobre os cenários analisados neste capítulo}

O cenário abordado considera uma rede interferente VSAT/TDMA servindo o território brasileiro, operando na faixa de 11-14 GHz (Banda Ku). A grande extensão geográfica do território brasileiro permite identificar regiões geográficas com diferentes densidades de terminais, considerada neste trabalho como proporcionais à distribuição populacional do Brasil. Assim, um elemento importante a ser considerado no modelo proposto é a demografia do território brasileiro. A Figura 4.1 apresenta a densidade demográfica do Brasil, fornecida pelo Instituto Brasileiro de Geografia e Estatística - IBGE [18].

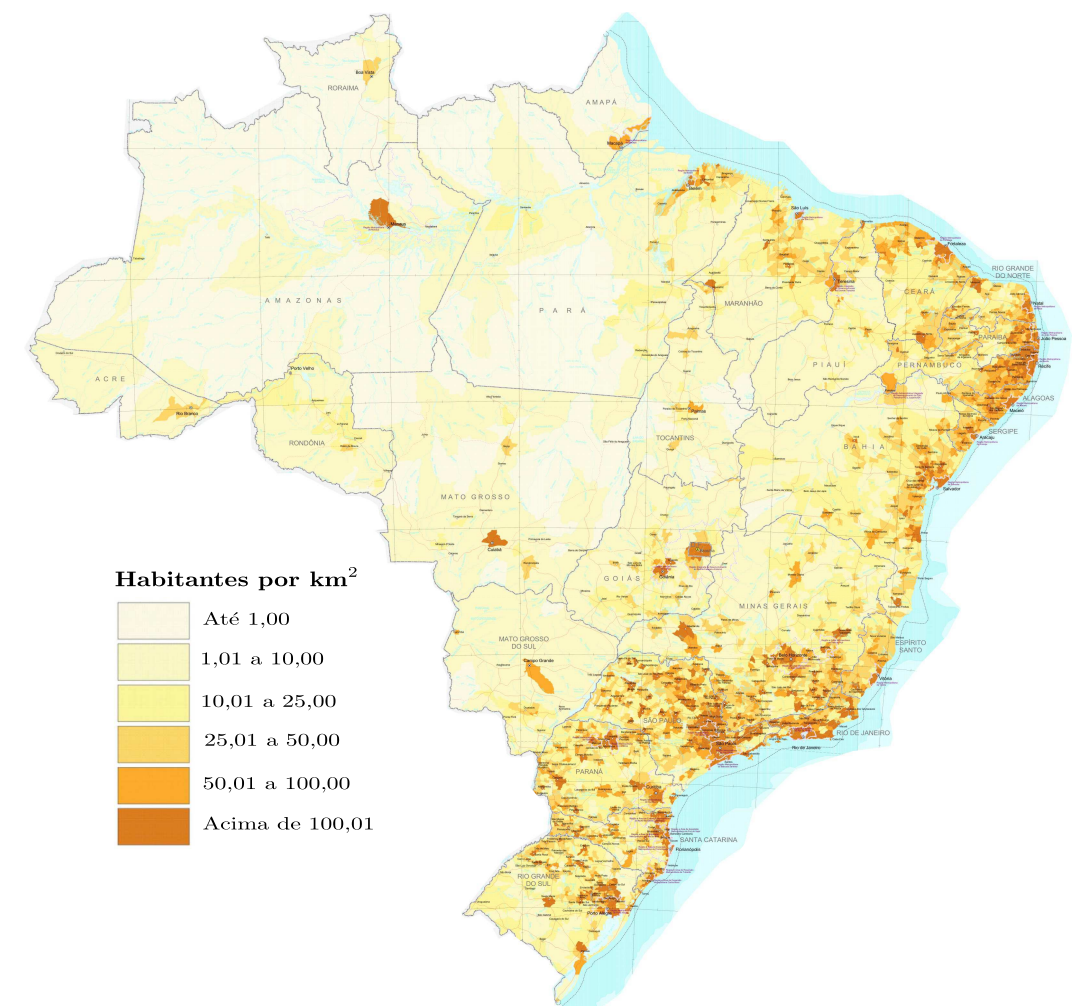

Figura 4.1: Mapa da densidade demográfica do Brasil [Habitantes $\left./ \mathrm{km}^{2}\right]$. Fonte: Instituto Brasileiro de Geografia e Estatística (IBGE)

Um outro dado importante na implementação do modelo proposto é a distribuição da taxa de precipitação pluvial no território brasileiro, que tem influência direta nos diâmetros das antenas dos terminais VSAT utilizados e, consequentemente, nas funções densidade de probabilidade da densidade de e.i.r.p. $p_{e_{\mathbf{r}} \mid \mathbf{r} \in \mathcal{R}_{m}}(E)$ das diferentes regiões consideradas. Neste trabalho, considerou-se a distribuição geográfica da taxa de precipitação (em milímetros por hora) excedida durante $0,01 \%$ da média anual, fornecida pela Recomendação ITU-R P.837-6 [15], ilustrada na Figura 4.2. 


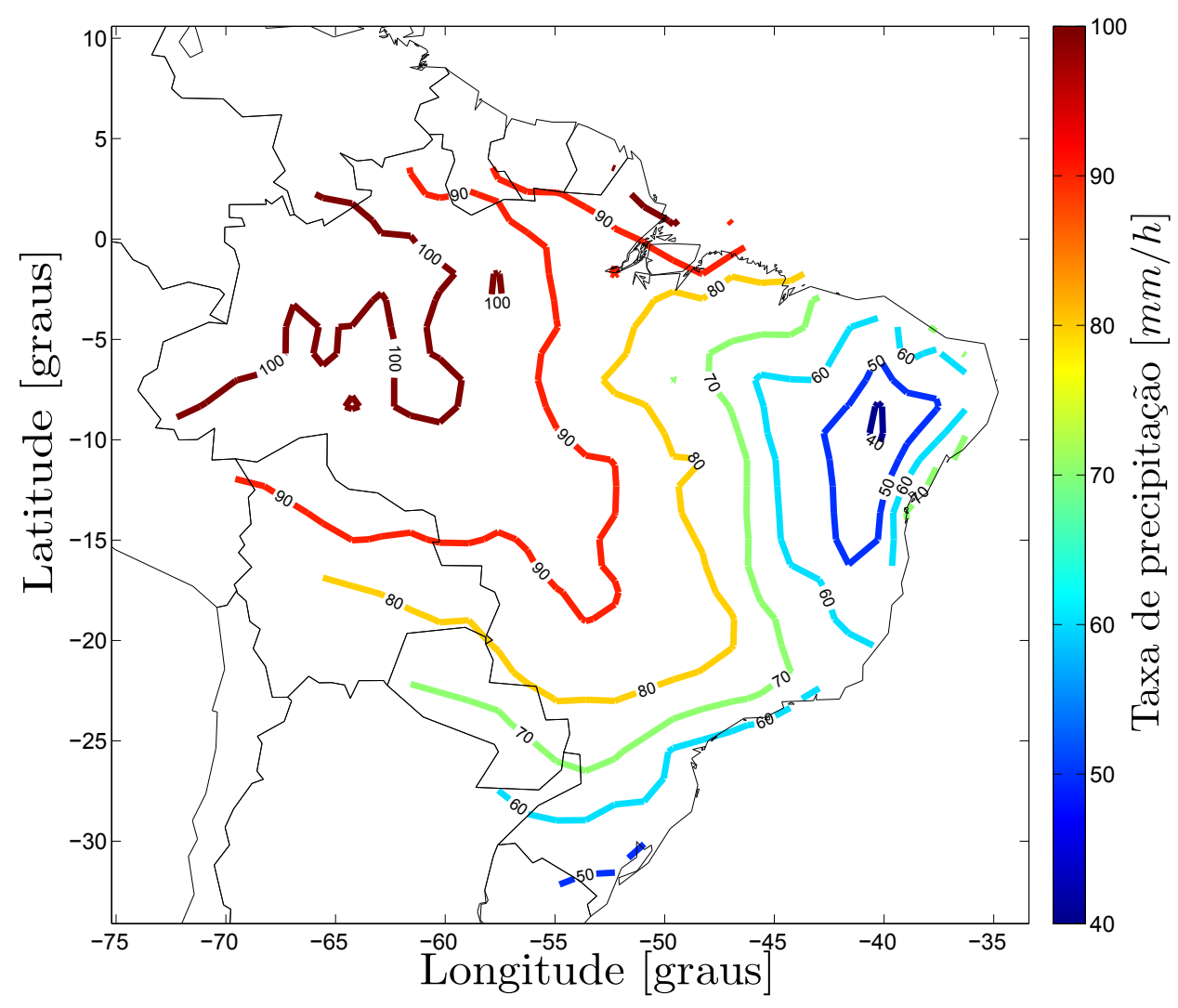

Figura 4.2: Taxa de precipitação pluvial no território brasileiro [mm/h].

\subsection{1 \\ Identificação de regiões}

Um primeiro passo, necessário para a aplicação do modelo matemático proposto, é a identificação, com base nas figuras 4.1 e 4.2 , de regiões no território brasileiro contendo uma densidade demográfica aproximadamente uniforme (ou com distribuição uniforme de clusters demográficos) e, ao mesmo tempo, pouca variação nas características de precipitação. Desse modo é possível considerar em cada uma dessas regiões, há uma distribuição de terminais bem definida (e.g. processo pontual de Poisson homogêneo com uma dada taxa de distribuição de pontos, ou processo pontual com clusters) e uma função densidade de probabilidade $p_{e_{\mathbf{r}} \mid \mathbf{r} \in \mathcal{R}_{m}}(E)$ válida para todos os pontos da região. Uma inspeção das figuras 4.1 e 4.2 resultou na identificação das seis regiões $(M=6)$ apresentadas na Figura 4.3.

As características de cada uma das regiões identificadas são apresentadas a seguir:

Região 1 corresponde à região leste da costa brasileira, a partir do Ceará, Rio Grande do Norte, Paraíba, Pernambuco, Alagoas, Sergipe, Bahia, Minas Gerais, Espírito Santo e Rio de Janeiro, com área de $747.075 \mathrm{~km}^{2}$. A taxa de precipitação pluvial é da ordem de 60 - $70 \mathrm{~mm} / \mathrm{h}$, sendo predominante 


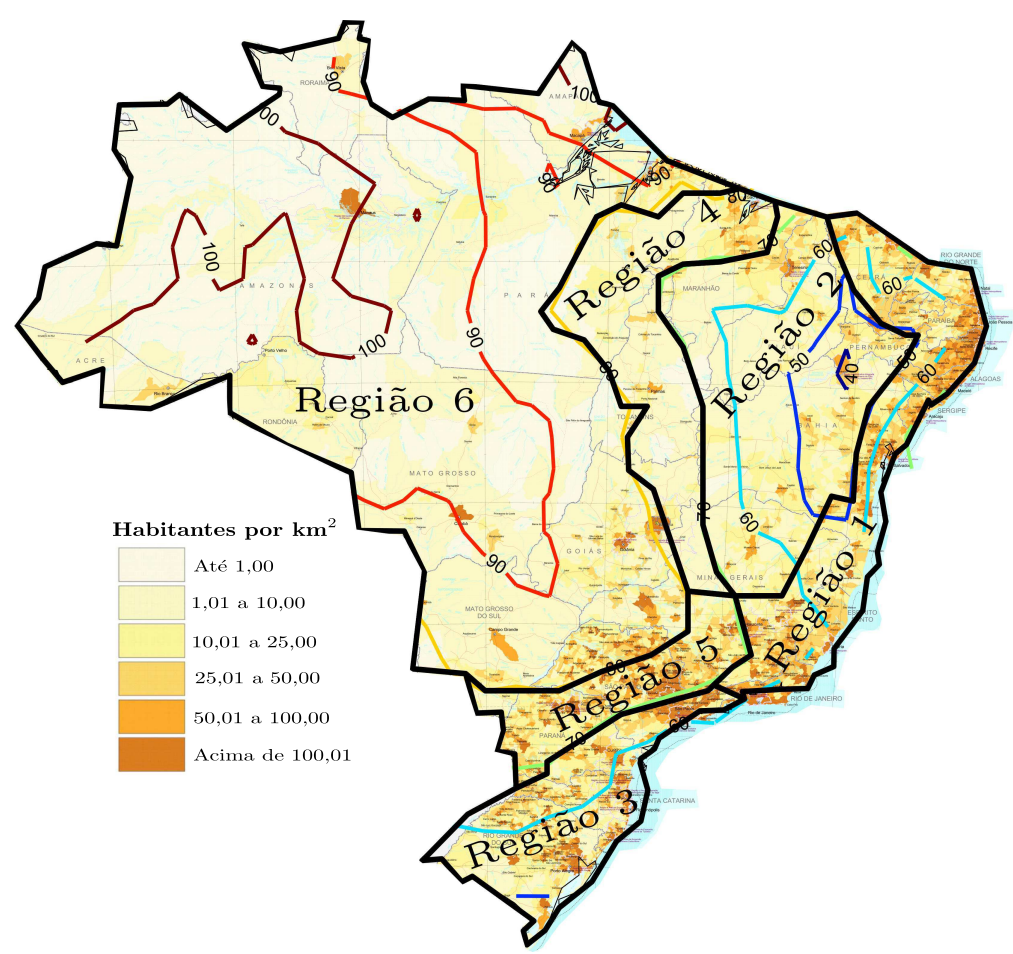

Figura 4.3: Taxa de precipitação pluvial $[\mathrm{mm} / \mathrm{h}]$ e Densidade demográfica [Habitantes por $\mathrm{km}^{2}$ ] do Brasil .

a taxa de $60 \mathrm{~mm} / \mathrm{h}$. A densidade demográfica pode ser considerada alta (entre 25 e 100 habitantes por $\mathrm{km}^{2}$ ) e, segundo o IBGE a região possui um total de 81.602.058 habitantes [19].

Região 2 encontra-se localizada na região centro leste do território brasileiro, constituída por a parte leste do estado de Maranhão, Piauí, parte sul este de Ceará, centro e leste de Pernambuco, centro e leste de Bahia, e norte de Minas Gerais, com uma extensão de $1.172 .461 \mathrm{~km}^{2}$. A taxa de precipitação pluvial é da ordem de 50 - $70 \mathrm{~mm} / \mathrm{h}$; sendo predominate as taxas de $60 \mathrm{~mm} / \mathrm{h}$ e $70 \mathrm{~mm} / \mathrm{h}$. A densidade demográfica pode ser considerada média (entre 1 e 25,1 habitantes por $\mathrm{km}^{2}$ ) e, segundo o IBGE a região possui um total de 6.600.495 habitantes [19].

Região 3 localizada na região sul do território brasileiro, constituída por parte da zona sul de Rio de Janeiro, São Paulo, parte leste de Paraná, Santa Catarina, e Rio Grande do Sul, com uma extensão de 486.807 $\mathrm{km}^{2}$. A taxa de precipitação pluvial é da ordem de $40-70 \mathrm{~mm} / \mathrm{h}$; sendo predominante a taxa de $60 \mathrm{~mm} / \mathrm{h}$. A densidade demográfica é considerada alta (entre 25 e 100 habitantes por $\mathrm{km}^{2}$ ), segundo IBGE a região possui um total de 63.121.589 habitantes [19].

Região 4 constituída por una estreita faixa localizada na região central do 
território brasileiro, conformada pela parte central e leste de Maranhão, parte leste de Pará, parte central e leste de Tocantins, leste e sul de Goiás, e leste de Minas Gerais, com uma extensão de $725.217 \mathrm{~km}^{2}$. A taxa de precipitação pluvial é da ordem de $60-70 \mathrm{~mm} / \mathrm{h}$. A densidade demográfica pode ser considerada média (entre 10 e 25 habitantes por $\mathrm{km}^{2}$ ), segundo o IBGE a região possui um total de 8.772 .292 habitantes [19].

Região 5 é conformada por parte do sul de Minas Gerais, centro de São Paulo, e leste de Paraná, com uma extensão de $335.342 \mathrm{~km}^{2}$. A taxa de precipitação pluvial é da ordem de 70 - 80 mm/h. A densidade demográfica é considerada como média alta (entre 25 e acima de 100 habitantes por $\mathrm{km}^{2}$ ) e, segundo o IBGE a região possui um total de 9.479.551 habitantes [19].

Região 6 é uma das regiões com maior extensão localizada na parte central, leste e nordeste do território brasileiro conformada por Rondônia, Acre, Amazonas, Roraima, centro e leste de Pará, Mato Grosso, Mato Grosso do Sul, centro e leste de Goiás, Distrito Federal, Amapá, leste e sul de Minas Gerais, e leste de São Paulo, com uma área de 5.101 .773 km². A taxa de precipitação pluvial é da ordem de $80-100 \mathrm{~mm} / \mathrm{h}$, considerada a mais alta do território brasileiro. A densidade demográfica pode ser considerada como baixa (entre 1 a 25 habitantes por $\mathrm{km}^{2}$ ). Observa-se nesta região clusters demográficos que são cidades com altas densidades demográficas (entre 25 e acima de 100 Habitantes por $\mathrm{km}^{2}$ ), caso de Manaus, Cuiabá, Campo Grande, Goiânia, Uberlândia, Brasilia e a região leste do estado de São Paulo. Segundo o IBGE a região possui um total de 34.874.664, habitantes [19].

\subsection{2}

\section{Atenuação por chuva e tamanhos de antenas}

Os cálculos usuais de enlace para redes VSAT/TDMA operando na Banda $\mathrm{Ku}$, indicam que antenas com diâmetros iguais a $0,96 \mathrm{~m}$ são usualmente utilizadas em terminais VSAT localizados em regiões onde a taxa de precipitação é da ordem de 40 a $60 \mathrm{~mm} / \mathrm{h}$. Terminais VSAT operando em regiões com taxa de precipitação da ordem de 60 a $80 \mathrm{~mm} / \mathrm{h}$ usualmente utilizam antenas com diâmetro 1,20 m. Antenas de diâmetro igual a 1,80 m são normalmente utilizadas nos terminais VSAT que operam em regiões com a taxa de precipitação da ordem 80 a 100 mm/h. Com base nessa informação, 
o presente trabalho considerou, para terminais operando em cada uma das seis regiões geográficas definidas e ilustradas na Figura 4.3, antenas com os diâmetros apresentados na Tabela 4.1.

Tabela 4.1: Taxa de precipitação e tamanho de antena por região

\begin{tabular}{|c|c|c|c|}
\hline \hline Região & $\begin{array}{c}\text { Taxa de precipi- } \\
\text { tação }[\mathrm{mm} / \mathrm{h}]\end{array}$ & $\begin{array}{c}\text { quantidade de dife- } \\
\text { rentes antenas }\left(N_{m}\right)\end{array}$ & $\begin{array}{c}\text { possíveis diâmetros de an- } \\
\text { tena }\left(d_{j}, j=1, \ldots, N_{m}\right)[\mathrm{m}]\end{array}$ \\
\hline $\mathcal{R}_{1}$ & $50-60$ & 1 & 0,96 \\
\hline $\mathcal{R}_{2}$ & $40-70$ & 2 & $0,96,1,20$ \\
\hline $\mathcal{R}_{3}$ & $50-70$ & 2 & $0,96,1,20$ \\
\hline $\mathcal{R}_{4}$ & $70-80$ & 2 & $1,20,1,80$ \\
\hline $\mathcal{R}_{5}$ & $70-80$ & 2 & $1,20,1,80$ \\
\hline $\mathcal{R}_{6}$ & $80-100$ & 1 & 1,80 \\
\hline \hline
\end{tabular}

Considera-se ainda que, em cada região, antenas com diferentes diâmetros têm a mesma probabilidade de serem utilizadas, ou seja, a probabilidade $P_{j m}$ de se utilizar uma antena com diâmetro $d_{j}$ na região $\mathcal{R}_{m}$ á dada por

$$
P_{j m}=\frac{1}{N_{m}}, \quad j=1, \ldots, N_{m}
$$

\subsection{3}

\section{Determinação da densidade de e.i.r.p. associada a cada tamanho de antena}

Neste trabalho, considerou-se um terminal VSAT utilizando uma antena de diâmetro $d_{j}$ que transmite a máxima densidade de e.i.r.p. permitida pela Recomendação ITU-R S.728 [12]. Esta recomendação limita a densidade de e.i.r.p. emitida fora do eixo de apontamento da antena (ângulo off-axis). Mais especificamente, no caso da Banda $\mathrm{Ku}$, esses limites, expressos em dB(W/40 $\mathrm{kHz}$ ), são dados por

$$
L_{728}(\varphi)= \begin{cases}(39-25 \log \varphi) & ; 2,5^{\circ} \leq \varphi \leq 7^{\circ} \\ 18 & ; 7^{\circ}<\varphi<9,2^{\circ} \\ (42-25 \log \varphi) & ; \quad 9.2^{\circ}<\varphi \leq 48^{\circ} \\ 0 & ; 48^{\circ}<\varphi \leq 180^{\circ}\end{cases}
$$

onde $\varphi$ representa o ângulo off-axis. O procedimento para determinar os valores das densidades de e.i.r.p. (na direção de máxima radiação das antenas) associados a cada um dos possíveis diâmetros de antena é descrito no Apêndice E. A aplicação deste procedimento resultou nos valores apresentados na Tabela 4.2 . 
Tabela 4.2: Valores de densidade de e.i.r.p., transmitidos pelos terminais VSAT na direção de máxima radiação das antenas.

\begin{tabular}{|c|c|}
\hline \hline $\begin{array}{c}\text { Diâmetro da antena } \\
\text { do terminal VSAT }[\mathrm{m}]\end{array}$ & $\begin{array}{c}\text { Densidade de e.i.r.p. } \\
{[\mathrm{dB}(\mathrm{W} / \mathrm{Hz})]}\end{array}$ \\
\hline 0,96 & $-3,42$ \\
\hline 1,20 & $-1,11$ \\
\hline 1,80 & 3,29 \\
\hline \hline
\end{tabular}

Em resumo, considerando-se a Tabela 4.1, as densidades de e.i.r.p. (na direção de máxima radiação das antenas) utilizadas pelos terminais VSAT em cada uma das regiões definidas são apresentadas na Tabela 4.3.

Tabela 4.3: Valores de $N_{m}$ e $E_{j m}$, correspondentes utilizados em cada uma das seis regiões geográficas ilustradas na Figura 4.3.

\begin{tabular}{|c|c|c|c|c|}
\hline \hline$m$ & \multirow{2}{*}{ Região $\mathcal{R}_{m}$} & \multirow{2}{*}{$N_{m}$} & \multicolumn{2}{|c|}{$E_{j m}, m=1, \ldots, N_{m}$} \\
\cline { 3 - 5 } & & $E_{1 m}$ & $E_{2 m}$ \\
\hline 1 & $\mathcal{R}_{1}$ & 1 & $-3,42$ & - \\
\hline 2 & $\mathcal{R}_{2}$ & 2 & $-3,42$ & $-1,11$ \\
\hline 3 & $\mathcal{R}_{3}$ & 2 & $-3,42$ & $-1,11$ \\
\hline 4 & $\mathcal{R}_{4}$ & 2 & $-1,11$ & 3,29 \\
\hline 5 & $\mathcal{R}_{5}$ & 2 & $-1,11$ & 3,29 \\
\hline 6 & $\mathcal{R}_{6}$ & 1 & 3,29 & - \\
\hline \hline
\end{tabular}

\subsection{4}

\section{Distribuição de terminais VSAT/MF-TDMA}

No cenário analisado neste capítulo, as posições geográficas dos terminais em cada uma das regiões consideradas foi modelada por um Processo Pontual de Poisson (Poisson Point Precess - PPP) homogêneo. Vale observar que, embora da distribuição demográfica da região $\mathcal{R}_{6}$ apresente a formação de clusters, a baixa densidade de terminais na região conduziu a uma distribuição de terminais também modelada por um PPP homogêneo (1 terminal por cluster demográfico e distribuição uniforme de clusters demográficos).

As densidades de terminais, $\lambda_{m}, m=1, \ldots, 6$ (terminais $/ \mathrm{km}^{2}$ ), em cada uma das regiões, foi determinada considerando-se o número total de terminais da rede $\mathcal{N}_{T}$ (dado conhecido que depende do tamanho da rede), a população $\mathcal{P}_{m}$ e a área $\mathcal{A}_{m}$ de cada uma das regiões. Inicialmente o número total de terminais $\mathcal{N}_{T}$ foi dividido entre as seis regiões considerando-se uma proporcionalidade entre o número de terminais $\mathcal{N}_{m}$ e a população $\mathcal{P}_{m}$ em cada região. Tem-se 
assim,

$$
\mathcal{N}_{m}=\frac{\mathcal{P}_{m}}{\sum_{m=1}^{M} \mathcal{P}_{m}} \mathcal{N}_{T} \quad ; \quad m=1, \ldots, M
$$

A densidade de terminais em cada região foi calculada dividindo-se o número de terminais na região por sua área, ou seja,

$$
\lambda_{m}=\frac{\mathcal{N}_{m}}{\mathcal{A}_{m}} ; \quad ; \quad m=1, \ldots, M
$$

ou ainda, considerando-se (4-6),

$$
\lambda_{m}=\frac{\mathcal{P}_{m}}{\mathcal{A}_{m} \sum_{m=1}^{M} \mathcal{P}_{m}} \mathcal{N}_{T} ; m=1, \ldots, M
$$

Os valores utilizados para $\mathcal{P}_{m}$ e $\mathcal{A}_{m}$ em cada uma das regiões, obtidos de [19], são apresentados na Tabela 4.4

Tabela 4.4: População $\mathcal{P}_{m}$ (habitantes) e Área $\mathcal{A}_{m}\left(\mathrm{~km}^{2}\right)$ correspondentes a cada região $\mathcal{R}_{m}$.

\begin{tabular}{|c|r|r|}
\hline \hline Região & População $\mathcal{P}_{m}$ & Área $\mathcal{A}_{m}\left[\mathrm{~km}^{2}\right]$ \\
\hline $\mathcal{R}_{1}$ & 81.602 .058 & 747.075 \\
\hline $\mathcal{R}_{2}$ & 6.600 .495 & 1.172 .461 \\
\hline $\mathcal{R}_{3}$ & 63.121 .589 & 486.807 \\
\hline $\mathcal{R}_{4}$ & 8.772 .292 & 725.217 \\
\hline $\mathcal{R}_{5}$ & 9.479 .551 & 335.342 \\
\hline $\mathcal{R}_{6}$ & 34.874 .664 & 5.101 .773 \\
\hline \hline
\end{tabular}

Em resumo, as densidades de terminais VSAT em cada uma das seis regiões $(M=6)$ identificadas na Figura 4.3 são apresentadas na Tabela 4.5.

Tabela 4.5: Densidade de terminais por região, para uma rede VSAT (inbound), $\operatorname{com} \mathcal{N}_{T}$ terminais.

\begin{tabular}{|c|c|}
\hline \hline Região & $\lambda_{m}, m=1, \ldots, 6$ [terminais $\left./ \mathrm{km}^{2}\right]$ \\
\hline $\mathcal{R}_{1}$ & $5,345 \times 10^{-7} \mathcal{N}_{T}$ \\
\hline $\mathcal{R}_{2}$ & $2,7535 \times 10^{-8} \mathcal{N}_{T}$ \\
\hline $\mathcal{R}_{3}$ & $6,34 \times 10^{-7} \mathcal{N}_{T}$ \\
\hline $\mathcal{R}_{4}$ & $5,915 \times 10^{-8} \mathcal{N}_{T}$ \\
\hline $\mathcal{R}_{5}$ & $1,3825 \times 10^{-7} \mathcal{N}_{T}$ \\
\hline $\mathcal{R}_{6}$ & $3,3435 \times 10^{-8} \mathcal{N}_{T}$ \\
\hline \hline
\end{tabular}

Note que, no caso de redes VSAT envolvendo regiões com diferentes características de distribuição de terminais, a determinação da função distribuição 
de probabilidade da densidade de potência interferente dada por (3-53), depende da probabilidade $P\left(\mathbf{r} \in \mathcal{R}_{m}\right)$ do canal TDMA estar sendo utilizado por um terminal da região $\mathcal{R}_{m}$ para $m=1, \ldots, M$. Estas probabilidades são aqui estimadas como a razão entre o número de terminais da rede em cada uma das regiões e o número total de terminais, ou seja

$$
P\left(\mathbf{r} \in \mathcal{R}_{m}\right)=\frac{\mathcal{N}_{m}}{\mathcal{N}_{T}} ; m=1, \ldots, M
$$

\subsection{5}

\section{Posições orbitais e antenas}

Neste trabalho considerou-se uma separação orbital entre os satélites interferente $S^{\prime}$ e vítima $S$ igual a $3,0^{\circ}\left(\Delta=3,0^{\circ}\right)$. Mais especificamente, o satélite interferente $S^{\prime}$ está localizado na posição orbital $65,0^{\circ} \mathrm{W}$, e o satélite vítima na posição orbital $62,0^{\circ} \mathrm{W}$. Além disso, considerou-se, para a antena de recepção do satélite vítima, um feixe circular cobrindo todo o território brasileiro e apontado para a posição $\left(54,9^{\circ} \mathrm{W}, 11,7^{\circ} \mathrm{S}\right)$. Esta geometria é ilustrada na Figura 4.4.

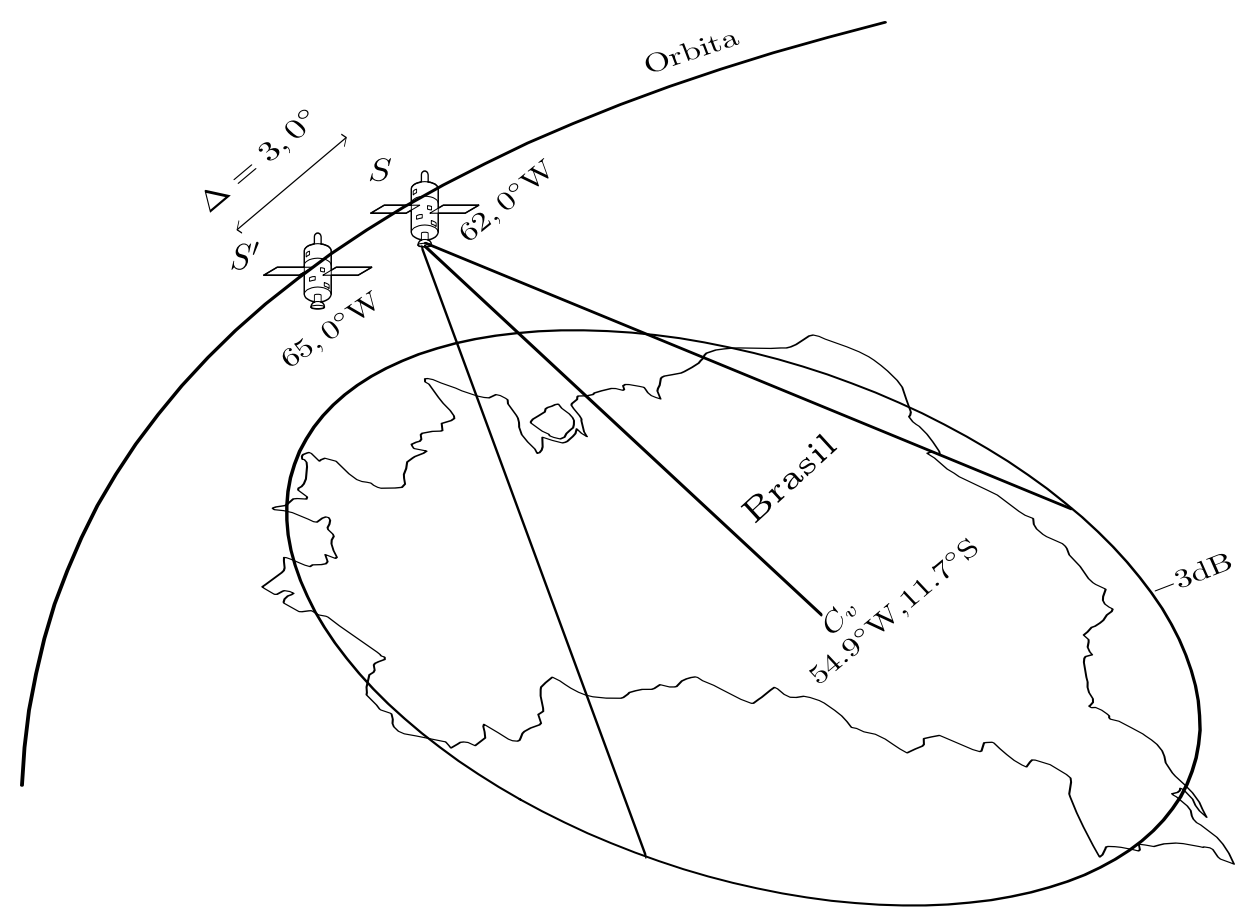

Figura 4.4: Separação orbital $\Delta$ (graus), entre satélites interferente $S^{\prime}$ e vítima $S$.

Neste cenário, assumiu-se que a estação terrena $R$ opera com uma antena receptora de alto ganho. Neste caso, o ganho $g_{4}(0)$ na direção do satélite $S$ é muito maior do que o ganho $g_{4}(\xi)$ na direção da rede do satélite interferente $S^{\prime}$, 
ou seja $g_{4}(0) \gg g_{4}(\xi)$, sendo então razoável supor que o ganho $\gamma$ associado com o satélite $S$ é muito maior do que $\gamma^{\prime}$ associado com o satélite $S^{\prime}$, ou seja $\gamma \gg \gamma^{\prime}$. Sob esta hipóteses, a densidade de potência interferente $i_{u}(\mathbf{r})$ é dominante, o que nos permite desprezar os efeitos da interferência $i_{d}(\mathbf{r})$ em (3-6), ou seja, $i(\mathbf{r}) \approx i_{u}(\mathbf{r})$. Neste caso

$$
i \approx \frac{e_{\mathbf{r}} g_{1 \mathbf{r}}^{\prime}\left(\theta_{\mathbf{r}}\right) g_{2}\left(\rho_{\mathbf{r}}\right)}{g_{1 \mathbf{r}}^{\prime}(0) l_{u \mathbf{r}}^{\prime \prime}} \gamma
$$

Para a antena receptora $g_{2}($ ) do satélite vítima (feixe circular) utilizou-se o diagrama de radiação da Recomendação ITU-R S.672 [14], dado por

$$
g_{2}(\Psi)= \begin{cases}G_{m}-3\left(\Psi / \Psi_{0}\right)^{2} & ; \Psi_{0} \leq \Psi<a \Psi_{0} \\ G_{m}+L_{s} & ; a \Psi_{0} \leq \Psi<b \Psi_{0} \\ G_{m}+L_{s}+20-25 \log \left(\Psi / \Psi_{0}\right) & ; b \Psi_{0} \leq \Psi<\Psi_{1} \\ 0 & ; \Psi_{1} \leq \Psi\end{cases}
$$

com $G_{m}=30,99 \mathrm{dBi}, \Psi_{0}=3^{\circ}, a=3,16, b=6,32$ e $L_{s}=-30 \mathrm{dBi}$. Este diagrama de radiação encontra-se ilustrado na Figura 4.5. Os contornos de cobertura produzidos por esta antena são apresentados na Figura 4.6.

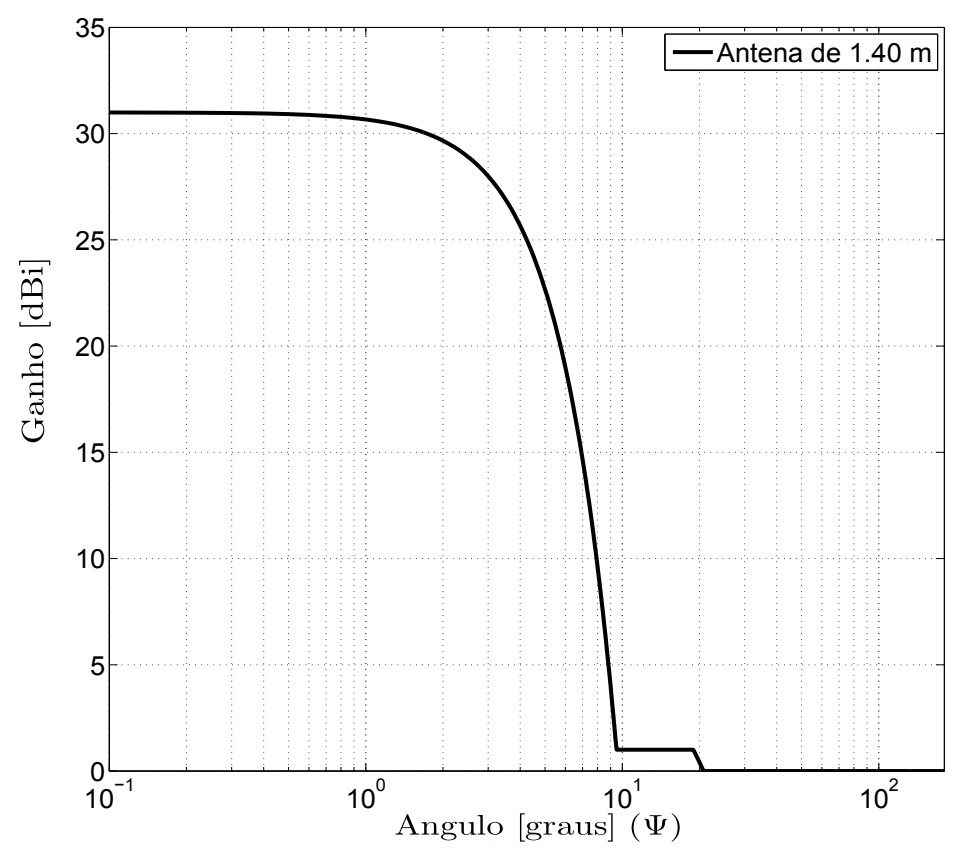

Figura 4.5: Diagrama de radiação da antena receptora do satélite vítima $S$.

Para as antenas transmissoras $g_{1 \mathbf{r}}^{\prime}()$ dos terminais VSAT (interferentes) utilizou-se o diagrama de radiação da Recomendação ITU-R F.1245-1 [13], 


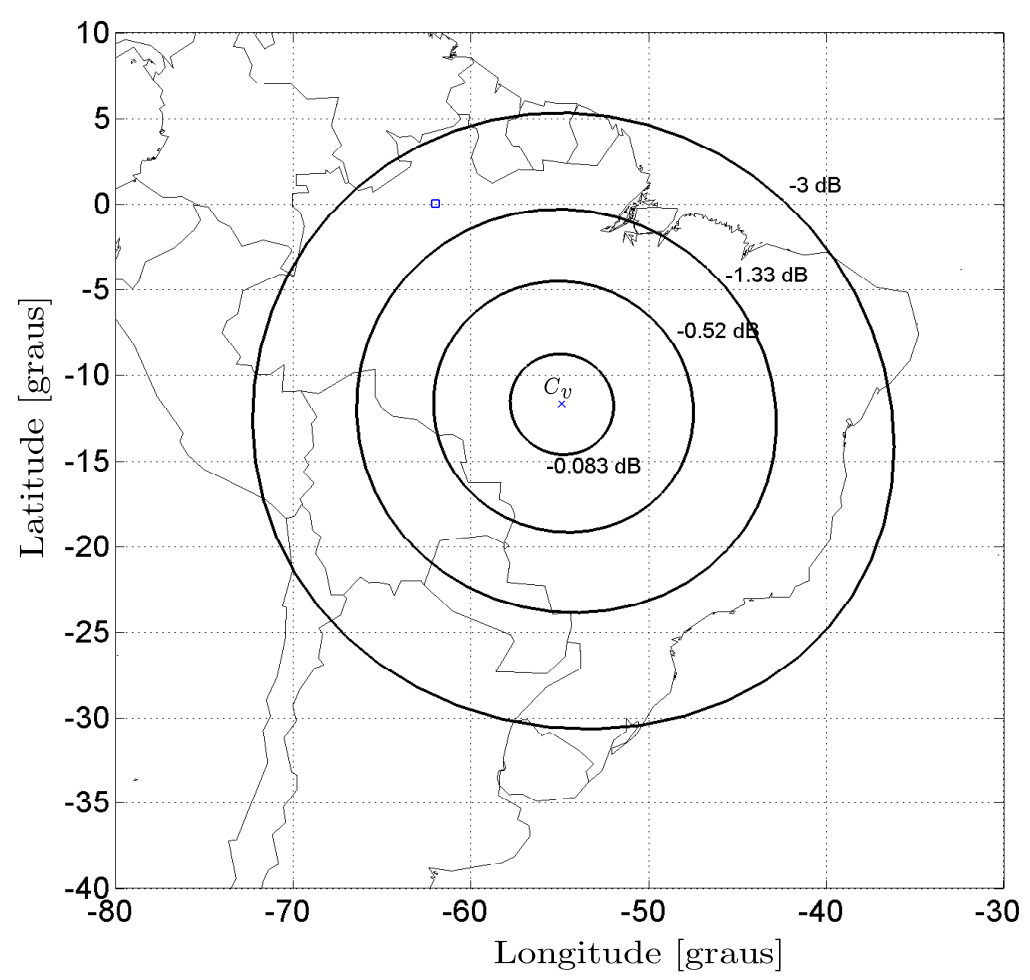

Figura 4.6: Contornos de cobertura da antena receptora do satélite vítima.

dado por

$$
g_{1 \mathbf{r}}^{\prime}(\varphi)= \begin{cases}G_{\max }-2,5 \times 10^{-3}\left(\frac{d}{\lambda} \varphi\right) & ; 0 \leq \varphi<\varphi_{m} \\ 39-5 \log (d / \lambda)-25 \log \varphi & ; \varphi_{m} \leq \varphi<48^{\circ} \\ -3-5 \log (d / \lambda) & ; \quad 48^{\circ} \leq \varphi<180^{\circ}\end{cases}
$$

onde $G_{\max }$ é o ganho da antena na direção de máxima radiação (em dBi), $d$ é o seu diâmetro, $\lambda$ é o comprimento de onda associado à frequência da transmissão e

$$
\varphi_{m}=\frac{20 \lambda}{d} \sqrt{G_{\max }-G_{1}}
$$

com

$$
G_{1}=2+15 \log (d / \lambda)
$$

Conforme mencionado anteriormente, dentro do cenário apresentado são considerados terminas VSAT com antenas de diâmetros $d_{j}$ iguais a 0,96, 1,20 e 1,80 metros, conforme indicado na Tabela 4.1. Valores de $G_{\max }, d / \lambda$ e $\varphi_{m}$ para antenas com cada um desses diâmetros são apresentados na Tabela 4.6. Os diagramas de radiação correspondentes são ilustrados na Figura 4.7.

Para os erros de apontamento (azimute $\phi_{a}$ e elevação $\phi_{e}$ ) das antenas dos terminais VSAT, modelados como variáveis aleatórias gaussianas com médias nulas e variâncias $\sigma^{2}$, considerou-se $\sigma^{2}=0,2$ (graus). 
Tabela 4.6: Valores de $G_{\max }, d / \lambda$ e $\varphi_{m}$ para antenas de diferentes diâmetros

\begin{tabular}{|c|c|c|c|}
\hline \hline$d[\mathrm{~m}]$ & $G_{\max }[\mathrm{dBi}]$ & $d / \lambda$ & $\varphi_{m}$ [graus] \\
\hline 0,96 & 40,37 & 44,80 & 1,65 \\
\hline 1,20 & 42,31 & 56,00 & 1,34 \\
\hline 1,80 & 45,83 & 84,00 & 0,92 \\
\hline \hline
\end{tabular}

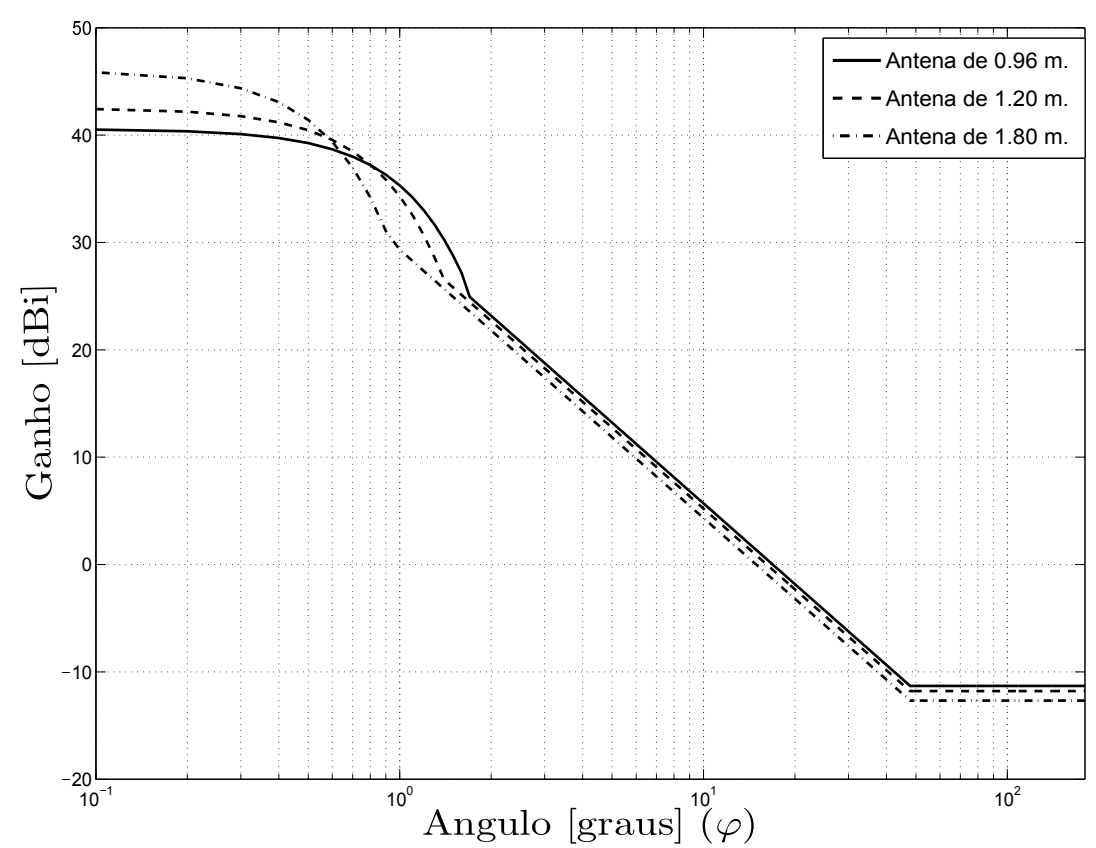

Figura 4.7: Diagrama de radiação das antenas transmissoras dos terminais VSAT. 


\section{2}

\section{Alguns exemplos específicos}

Dentro do cenário definido na Seção 4.1, são analisados quatro exemplos que abordam redes VSAT/TDMA cobrindo todo o território brasileiro. Os três primeiros analisam situações envolvendo regiões geográficas com um único tipo de distribuição de terminais, mais especificamente, com distribuição modelada por um Processo Pontual de Poisson Homogêneo bidimensional (PPP). Nestes três exemplos são abordadas, respectivamente, situações com densidades alta, media e baixa de terminais VSAT. Resultados correspondentes a esses três exemplos são apresentados na Seção 4.2.1. No quarto exemplo, descrito na Seção 4.2.2, é considerada uma situação envolvendo terminais VSAT distribuídos ao longo de uma área geográfica contendo múltiplos tipos de distribuição de terminais. Em cada um desses quatro exemplos são consideradas duas redes VSAT, aqui denominadas Rede 1 e Rede 2, com diferentes números de terminais. O número de terminais dessas redes foi escolhido de modo a possibilitar a comparação dos resultados obtidos com o modelo proposto com aqueles resultantes de dados reais (posições dos terminais, diâmetros das antenas, e e.i.r.p associados a cada antena) fornecidos pela operadora de satélites Embratel Star One.

\subsection{1}

\section{Regiões com um único tipo de distribuição de terminais}

Nesta subseção, são consideradas redes VSAT interferentes atuando numa área de serviço com um único tipo de distribuição de terminais VSAT. Mais especificamente, são consideradas redes com distribuição uniforme de terminais. As posições geográficas dos terminais são aqui modeladas por processos pontuais de Poisson homogêneos (densidade média de terminais $\lambda$ constante). Além disso, considera-se, nesta subseção, que a função densidade de probabilidade da densidade de e.i.r.p. é dada por (3-34), ou seja, é a mesma para todos os pontos da área de serviço. Neste caso, a função distribuição de probabilidade da densidade de potência interferente é determinada utilizandose (3-38) e (3-41).

São apresentados três exemplos, envolvendo diferentes áreas de serviço e diferentes densidades de terminais. Os resultados relativos a cada um desses exemplos são apresentados nas subseções 4.2.1.1 a 4.2.1.2. 


\subsubsection{1}

\section{Exemplo envolvendo a Região $\mathcal{R}_{1}$}

Neste exemplo considera-se que a Rede 1 tem 67 terminais VSAT $\left(\mathcal{N}_{T}=67\right)$ e a Rede 2 possui 18 terminais $\operatorname{VSAT}\left(\mathcal{N}_{T}=18\right)$. Estes valores foram escolhidos de modo a possibilitar a comparação dos resultados obtidos com o modelo proposto com aqueles resultantes de dados reais fornecidos pela operadora de satélites Embratel Star One. Levando-se em conta que a área da Região $\mathcal{R}_{1}$ é de $747.075 \mathrm{~km}^{2}$, esses números de terminais conduzem aos valores de densidade de $8,96 \times 10^{-5}$ terminais por $\mathrm{km}^{2}$ e $2,40 \times 10^{-5}$ terminais por $\mathrm{km}^{2}$ para as redes 1 e 2 , respectivamente.

Com base nos comentários da Seção 4.1.2, considerou-se ainda que, em ambas as redes, os terminais VSAT operam com um único tamanho de antena, correspondente a 0.96 metros $\left(N_{1}=1, P_{11}=1\right)$. Para a densidade de e.i.r.p. transmitida pelos terminais VSAT considerou-se o valor apresentado na Tabela 4.2 , ou seja, $E_{11}=-3,42 \mathrm{~dB}(\mathrm{~W} / \mathrm{Hz})$.

As figuras 4.8 e 4.9 ilustram, respectivamente, distribuições típicas (amostras de um PPP) de terminais VSAT das redes 1 e 2 na Região $\mathcal{R}_{1}$. Estas figuras incluem ainda a posição geográfica $R_{r e f}$ utilizada no cálculo do valor de referência $i_{\text {ref }}$.

O comportamento estatístico (função distribuição de probabilidade complementar) da variável aleatória $i_{n_{\mathrm{dB}}}$, que caracteriza a densidade de potência interferente produzida pelos terminais VSAT da Rede 1 no enlace vítima, é apresentado na Figura 4.10 (linha cheia). A título de comparação, esta figura inclui ainda estimativas da função distribuição de probabilidade complementar de $i_{n_{\mathrm{dB}}}$, obtidas diretamente de dados reais fornecidos pela operadora de satélites Embratel Star One, considerando-se quatro diferentes conjuntos de valores de erro de apontamento de antenas escolhidos aleatoriamente (pontos ligados por linhas). No caso de dados reais, esta figura inclui ainda a curva média (linha tracejada) obtida a partir de diferentes conjuntos de valores de erro de apontamento das antenas escolhidos aleatoriamente.

De maneira análoga, o comportamento estatístico da variável aleatória $i_{n_{\mathrm{dB}}}$, que caracteriza a densidade de potência interferente produzida pelos terminais VSAT da Rede 2 no enlace vítima, é apresentado na Figura 4.11 (linha cheia). Novamente, a título de comparação, esta figura inclui estimativas obtidas diretamente de dados reais fornecidos pela Embratel Star One, considerando-se quatro diferentes conjuntos de valores de erro de apontamento das antenas escolhidos aleatoriamente (pontos ligados por linhas). No caso de dados reais, esta figura também inclui ainda a curva média (linha tracejada) obtida a partir de diferentes conjuntos de valores de erro de apontamento das 


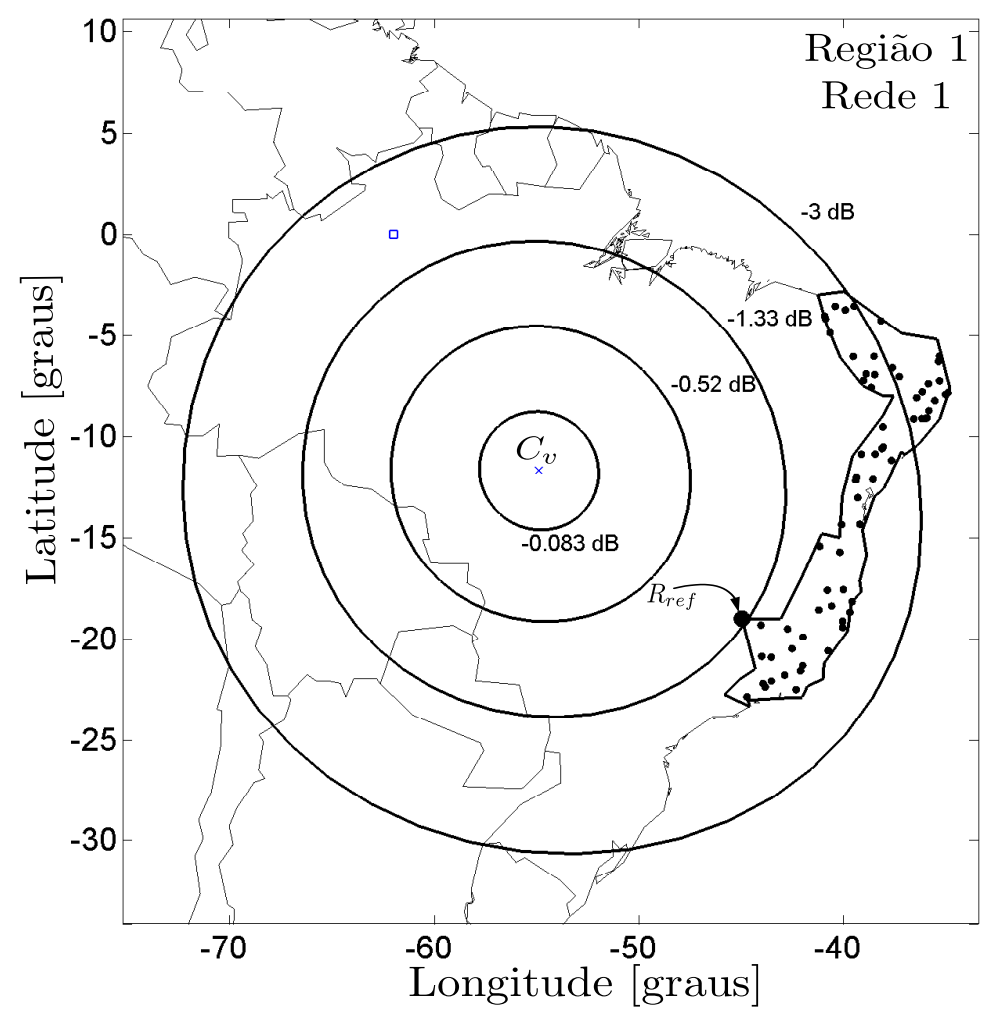

Figura 4.8: Amostra típica da distribuição de terminais da Rede 1 na Região 1 (PPP com densidade média de $8,96 \times 10^{-5}$ terminais por $\mathrm{km}^{2}$ ). Inclui a posição geográfica $R_{\text {ref }}$ utilizada no cálculo do valor de referência $i_{r e f}$ $\left(44,96^{\circ} \mathrm{W}, 19,01^{\circ} \mathrm{S}\right)$.

antenas escolhidos aleatoriamente.

Note em ambos os casos abordados neste exemplo (redes 1 e 2), que em torno de $\mathrm{I}[\mathrm{dB}]=0$, existe uma concordância bastante grande entre os resultados produzidos pelo modelo proposto e a curva média obtidos com base em dados reais.

Observe que, no caso dos dados reais (ver curvas com pontos ligados por linhas nas figuras 4.10 e 4.11), a aleatoriedade esta relacionada com a escolha do terminal $T^{\prime}$ que vai transmitir em um determinado canal em um dado instante. E, considerou-se que os terminais VSAT têm a mesma probabilidade de ser escolhido.

Note ainda que, conforme esperado, por utilizar um modelo analítico na caracterização do comportamento estatístico do ângulo $\theta_{\mathbf{r}}$, o modelo proposto é capaz de fornecer a função distribuição de probabilidade de $i_{n_{\mathrm{dB}}}$ mesmo para valores bastante ínfimos de $P\left(i_{n_{\mathrm{dB}}}>I\right.$ ) (nas figuras estas probabilidade estão limitadas inferiormente por $10^{-5}$ ). Já as estimativas obtidas a partir de dados reais dependem da quantidade de dados considerados (basicamente do número de terminais da rede), conforme refletido nos resultados apresentados nas figuras 4.10 (67 terminais) e 4.11 (18 terminais). 


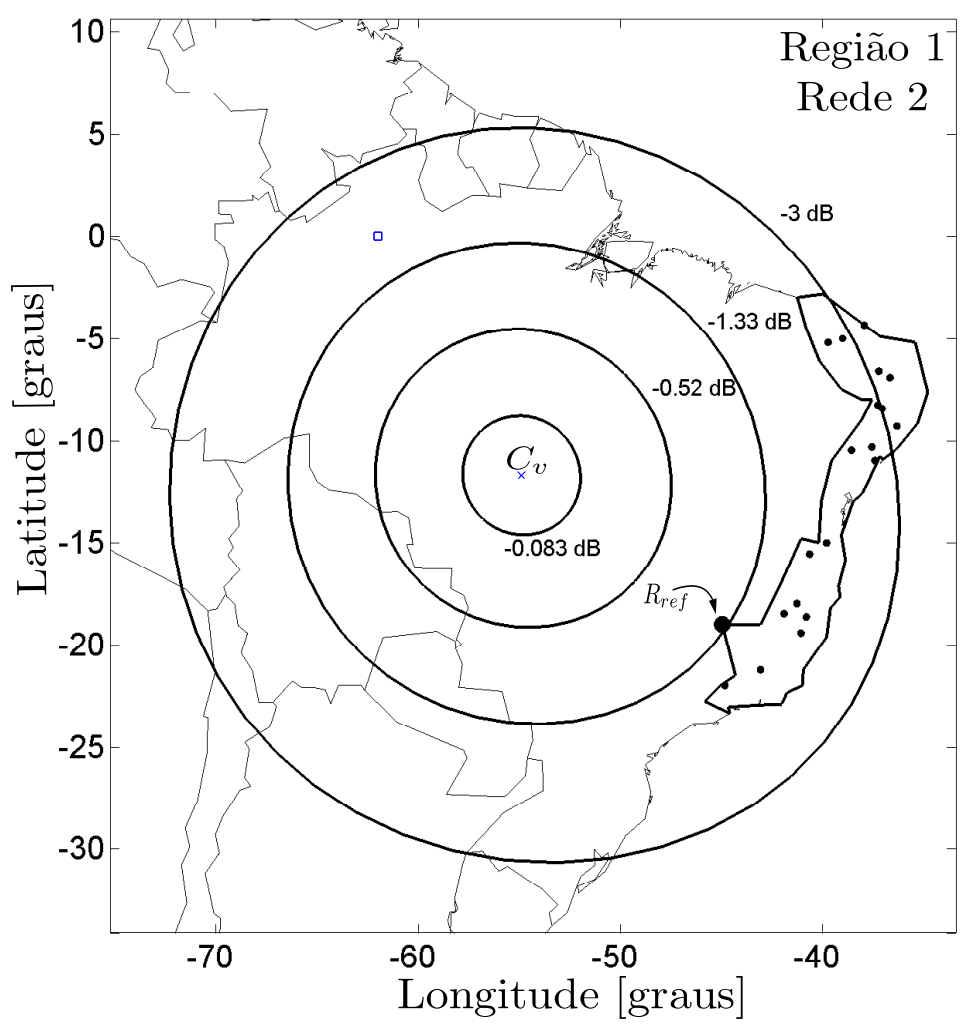

Figura 4.9: Amostra típica da distribuição de terminais da Rede 2 na Região 1 (PPP com densidade média de $2,40 \times 10^{-5}$ terminais por $\mathrm{km}^{2}$ ). Inclui a posição geográfica $R_{r e f}$ utilizada no cálculo do valor de referência $i_{r e f}$ $\left(44,96^{\circ} \mathrm{W}, 19,01^{\circ} \mathrm{S}\right)$. 


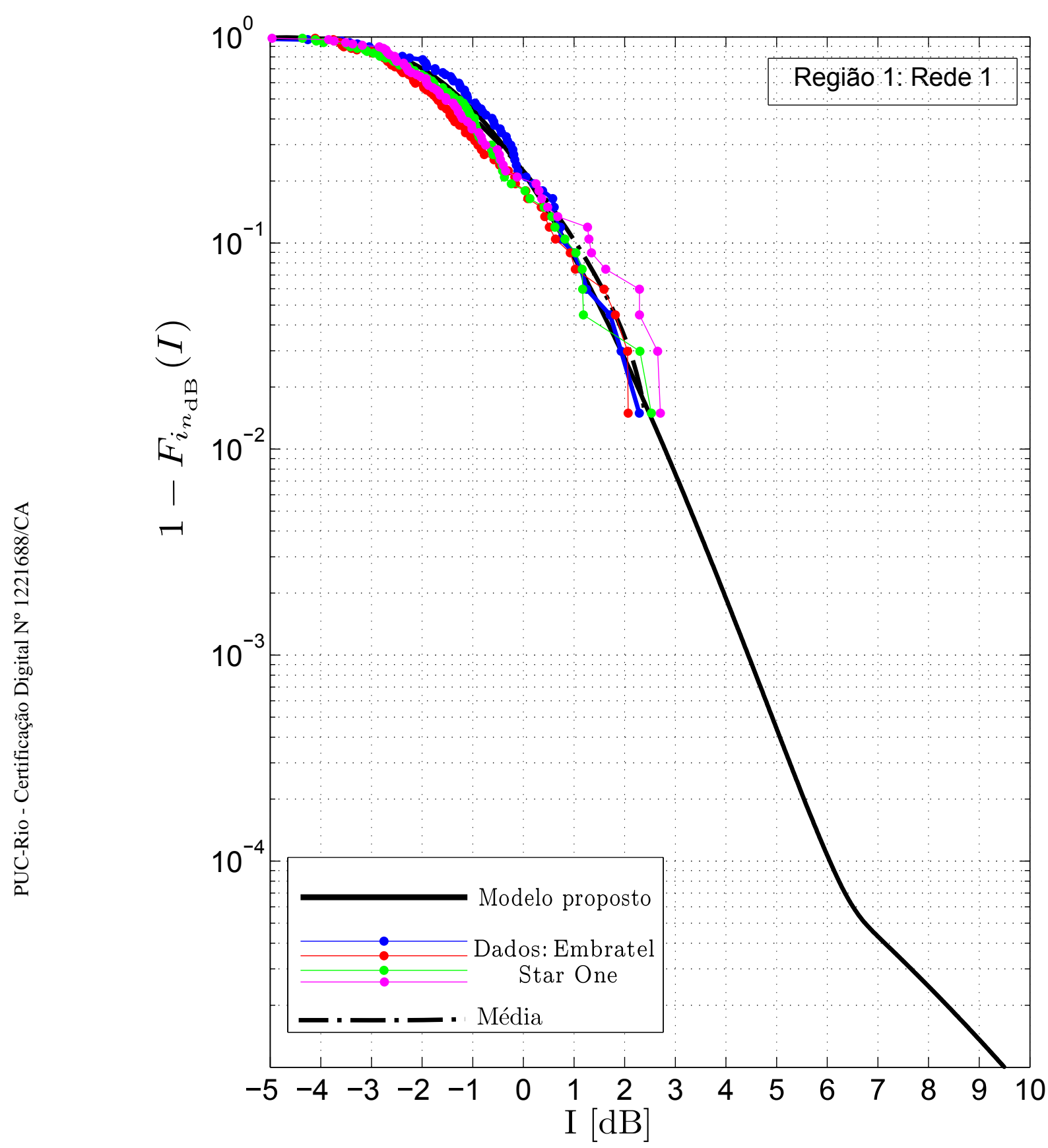

Figura 4.10: Função distribuição de probabilidade complementar correspondente à Rede 1, com 67 terminais VSAT na Região 1. 


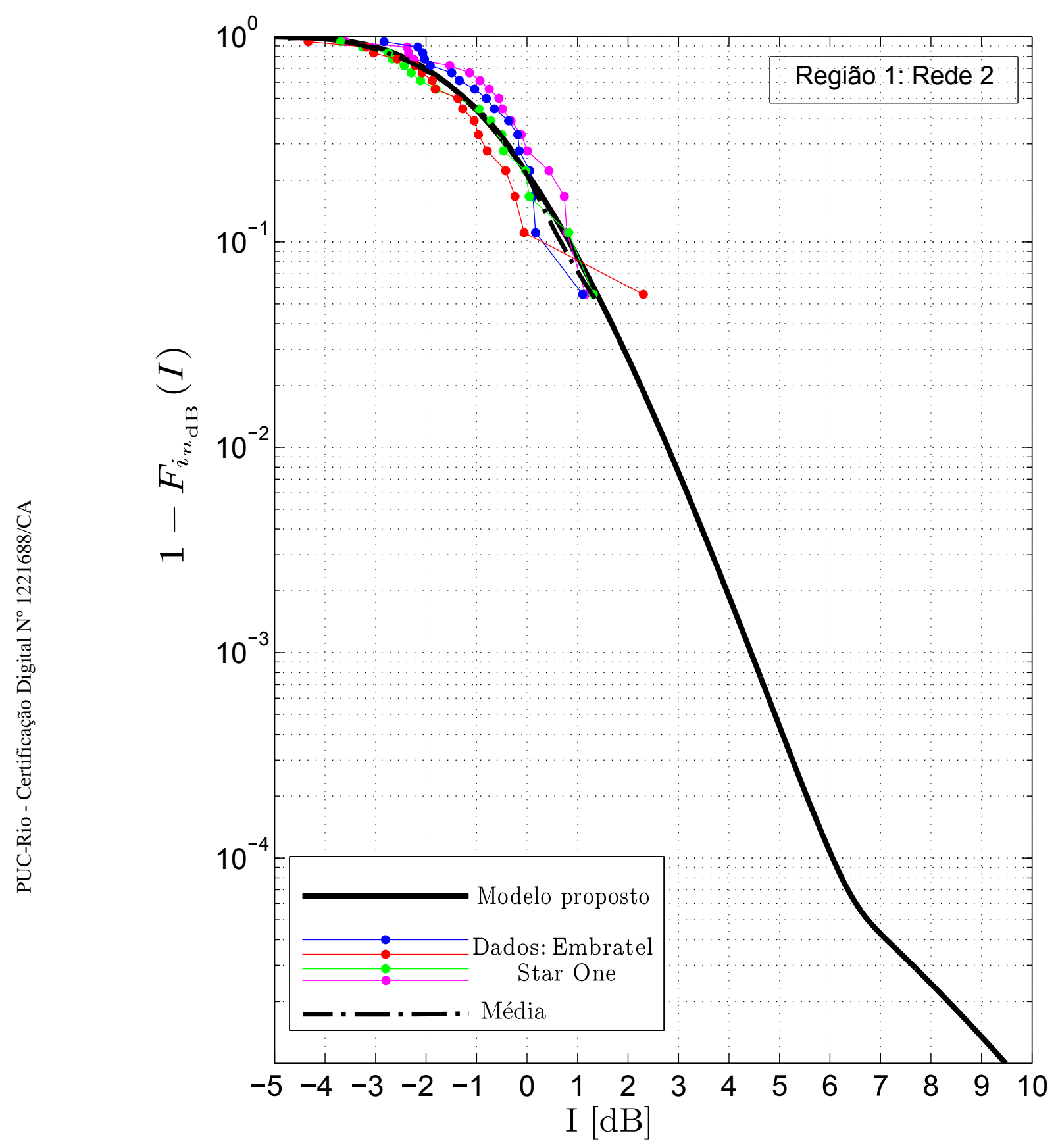

Figura 4.11: Função distribuição de probabilidade complementar correspondente à Rede 2, com 18 terminais VSAT na Região 1. 


\subsubsection{2}

\section{Exemplo envolvendo a Região $\mathcal{R}_{6}$}

Este exemplo tem como objetivo ilustrar o comportamento do modelo proposto em situações envolvendo densidades de terminais mais baixas, o que ocorre na região $\mathcal{R}_{6}$. Considera-se que a Rede 1 tem 85 terminais $\operatorname{VSAT}\left(\mathcal{N}_{T}=\right.$ $85)$ e a Rede 2 possui 26 terminais $\operatorname{VSAT}\left(\mathcal{N}_{T}=26\right)$. Novamente, estes valores foram escolhidos de modo a possibilitar a comparação dos resultados obtidos com o modelo proposto com aqueles resultantes de dados reais fornecidos pela Embratel Star One. Levando-se em conta que a área da Região $\mathcal{R}_{6}$ é de $5.101 .773 \mathrm{~km}^{2}$, esses números de terminais conduzem aos valores de densidade $1,66 \times 10^{-5}$ terminais por $\mathrm{km}^{2}$ e $5,09 \times 10^{-6}$ terminais por $\mathrm{km}^{2}$ para as redes 1 e 2 , respectivamente.

Com base nos comentários da Seção 4.1, considerou-se ainda que, em ambas redes, os terminais VSAT operam com um tamanho de antena, correspondente a 1,80 metros $\left(N_{1}=1, P_{16}=1\right)$. Para a densidade de e.i.r.p. transmitida pelos terminais VSAT considerou-se o valor apresentado na Tabela 4.2 , ou seja, $E_{16}=3,29 \mathrm{~dB}(\mathrm{~W} / \mathrm{Hz})$.

As figuras 4.12 e 4.13 ilustram, respectivamente, distribuições típicas (amostras de um PPP) de terminais VSAT das redes 1 e 2 na Região $\mathcal{R}_{6}$. Estas figuras incluem ainda a posição geográfica $R_{\text {ref }}$ utilizada no cálculo do valor de referência $i_{r e f}$.

O comportamento estatístico (função distribuição de probabilidade complementar) da variável aleatória $i_{n_{\mathrm{dB}}}$, que caracteriza a densidade de potência interferente produzida pelos terminais VSAT da Rede 1 no enlace vítima, é apresentado na Figura 4.14 (linha cheia). A título de comparação, esta figura inclui ainda estimativas da função distribuição de probabilidade complementar de $i_{n}$, obtidas diretamente de dados reais fornecidos operadora de satélites Embratel Star One, considerando-se quatro diferentes conjuntos de valores de erro de apontamento de antenas escolhidos aleatoriamente (pontos ligados por linhas). No caso de dados reais, esta figura inclui ainda a curva média (linha tracejada) obtida a partir de diferentes conjuntos de valores de erro de apontamento das antenas escolhidos aleatoriamente.

De maneira análoga, o comportamento estatístico da variável aleatória $i_{n_{\mathrm{dB}}}$, que caracteriza a densidade de potência interferente produzida pelos terminais VSAT da Rede 2 no enlace vítima, é apresentado na Figura 4.15 (linha cheia). Novamente, a título de comparação, esta figura inclui estimativas obtidas diretamente de dados reais fornecidos operadora de satélites Embratel Star One, considerando-se quatro diferentes conjuntos de valores de erro de apontamento de antenas escolhidos aleatoriamente (pontos ligados por linhas). 


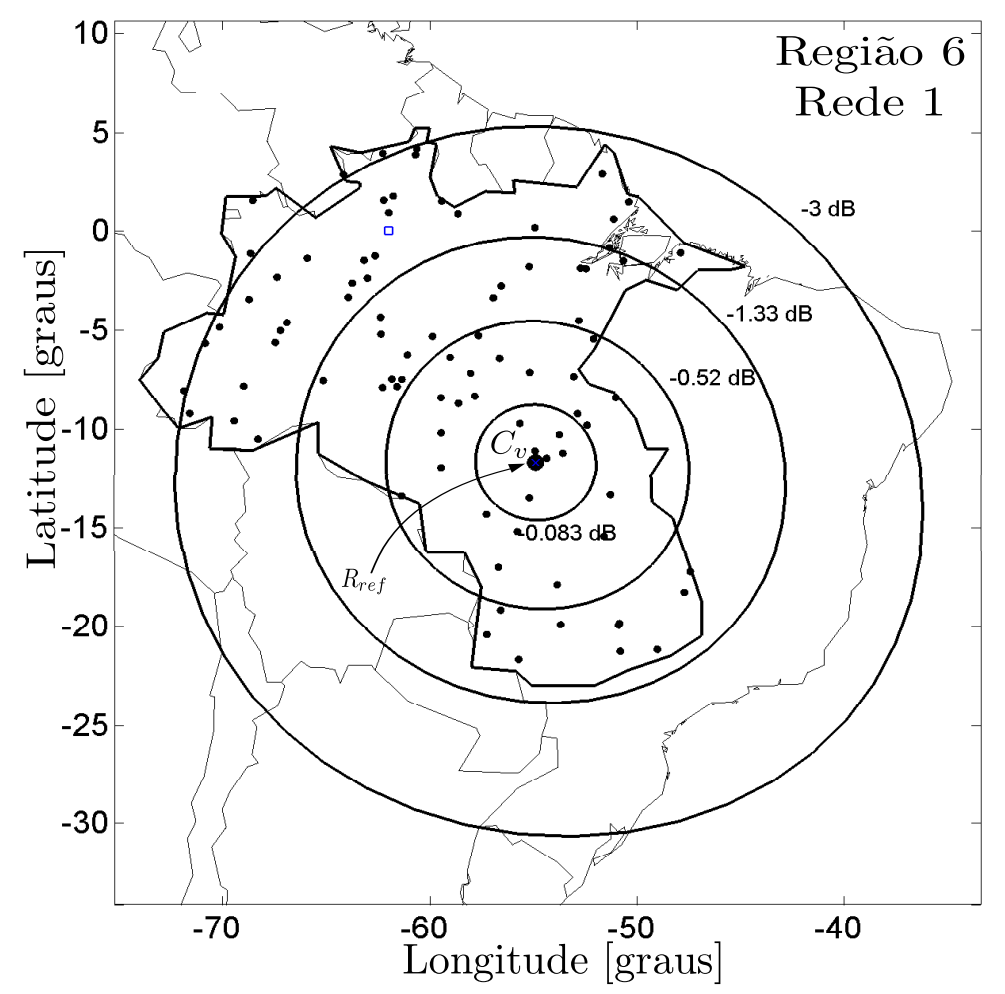

Figura 4.12: Amostra típica da distribuição de terminais da Rede 1 na Região 6 (PPP com densidade média de $1,66 \times 10^{-5}$ terminais por $\mathrm{km}^{2}$ ). Inclui a posição geográfica $R_{\text {ref }}$ utilizada no cálculo do valor de referência $i_{r e f}$ $\left(54,90^{\circ} \mathrm{W}, 11,70^{\circ} \mathrm{S}\right)$.

No caso de dados reais, esta figura também inclui ainda a curva média (linha tracejada) obtida a partir de diferentes conjuntos de valores de erro de apontamento das antenas escolhidos aleatoriamente.

Observe neste exemplo que em torno de $\mathrm{I}[\mathrm{dB}]=0$, existe uma diferença aceitável de aproximadamente $0,85 \mathrm{~dB}$ (rede 1) e 0,70 dB (rede 2), entre os resultados produzidos pelo modelo proposto e a curva média obtidos com base em dados reais, sendo o modelo proposto mais conservador.

No caso dos dados reais (ver curvas com pontos ligados por linhas nas figuras 4.14 e 4.15), a aleatoriedade esta relacionada com a escolha do terminal $T^{\prime}$ que vai transmitir em um determinado canal em um dado instante. E, considerou-se que os terminais VSAT têm a mesma probabilidade de ser escolhido.

Note ainda que, conforme esperado, por utilizar um modelo analítico na caracterização do comportamento estatístico do ângulo $\theta_{\mathbf{r}}$, o modelo proposto é capaz de fornecer a função distribuição de probabilidade de $i_{n_{\mathrm{dB}}}$ mesmo para valores bastante ínfimos de $P\left(i_{n_{\mathrm{dB}}}>I\right.$ ) (nas figuras estas probabilidade estão limitadas inferiormente por $10^{-5}$ ). Já as estimativas obtidas a partir de dados reais dependem da quantidade de dados considerados (basicamente do 


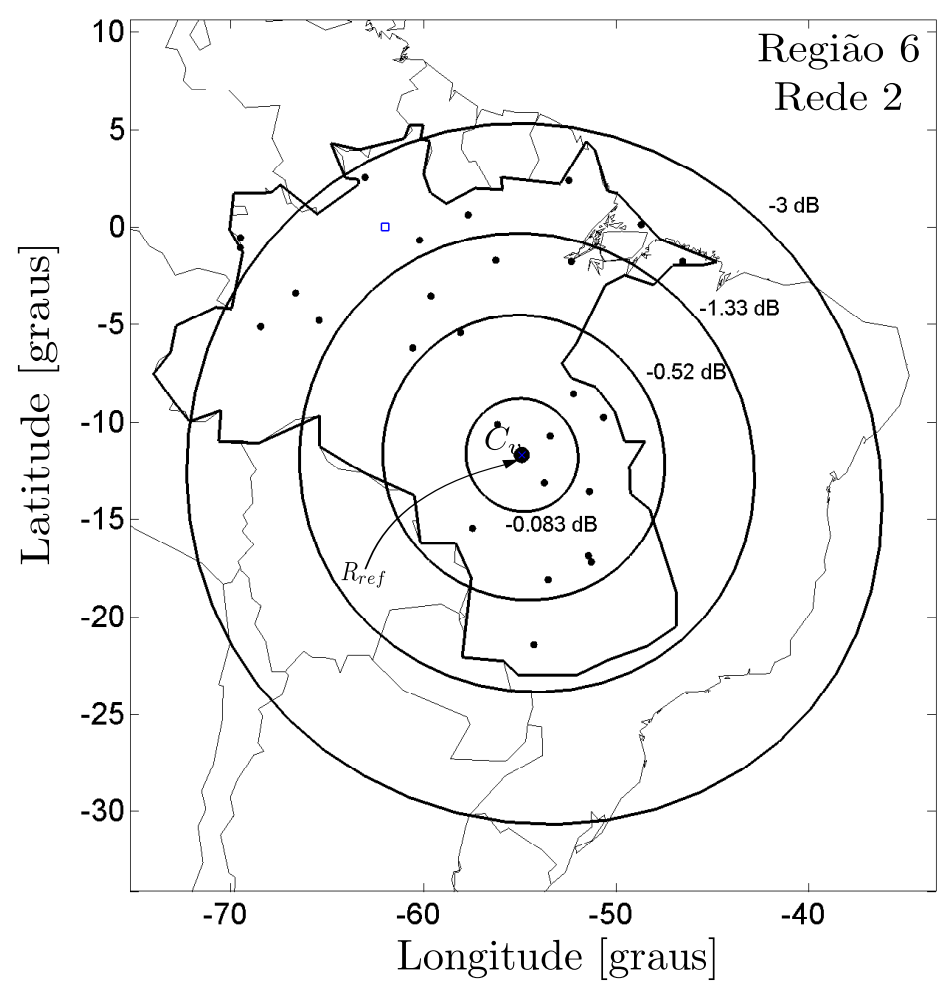

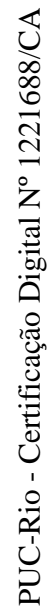

Figura 4.13: Amostra típica da distribuição de terminais da Rede 2 na Região 6 (PPP com densidade média de $5,09 \times 10^{-6}$ terminais por $\mathrm{km}^{2}$ ). Inclui a posição geográfica $R_{\text {ref }}$ utilizada no cálculo do valor de referência $i_{\text {ref }}$ $\left(54,90^{\circ} \mathrm{W}, 11,70^{\circ} \mathrm{S}\right)$.

número de terminais da rede), conforme refletido nos resultados apresentados nas figuras 4.14 (85 terminais) e 4.15 (26 terminais). 


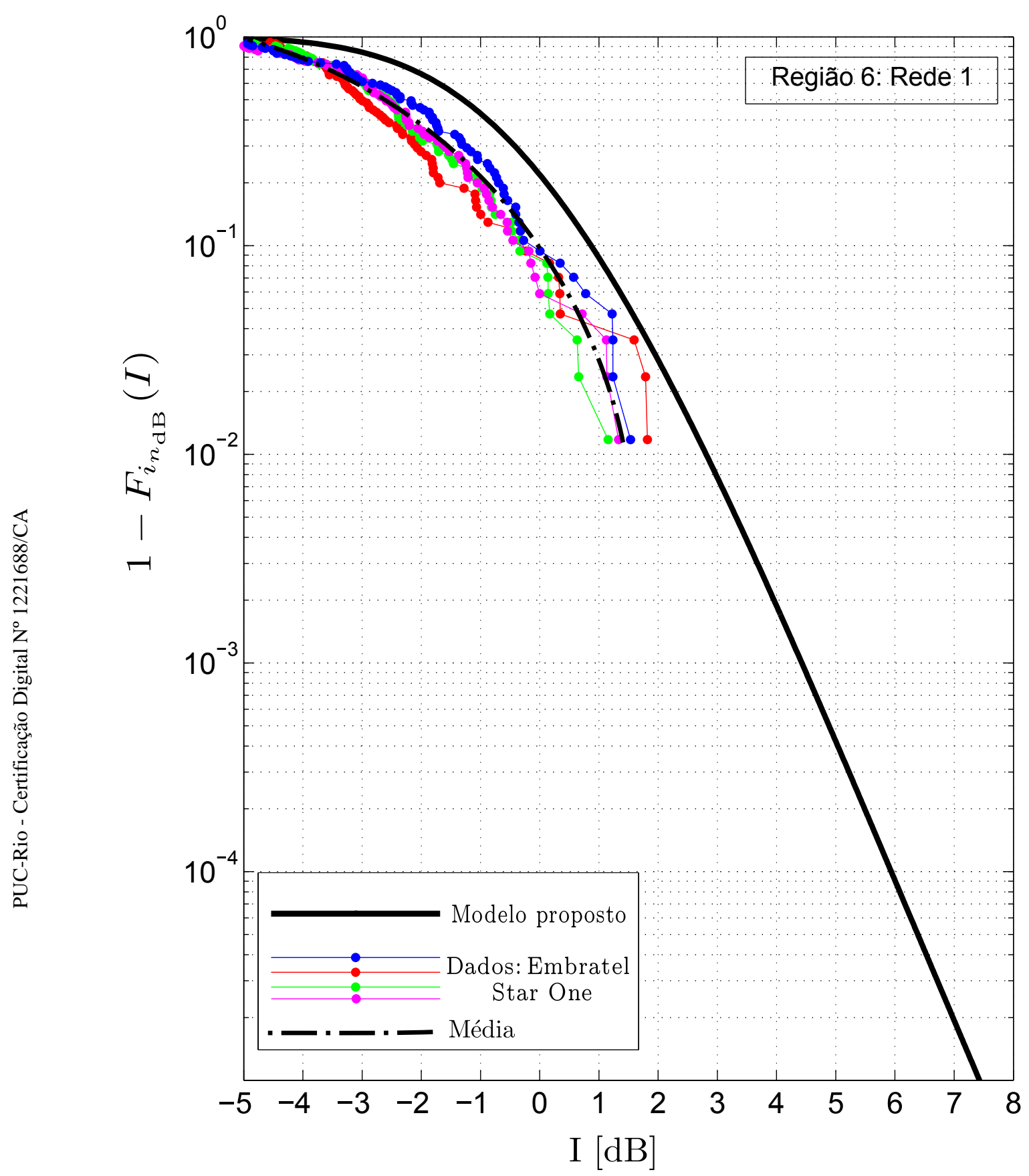

Figura 4.14: Função distribuição de probabilidade complementar correspondente à Rede 1, com 85 terminais VSAT na Região 6. 


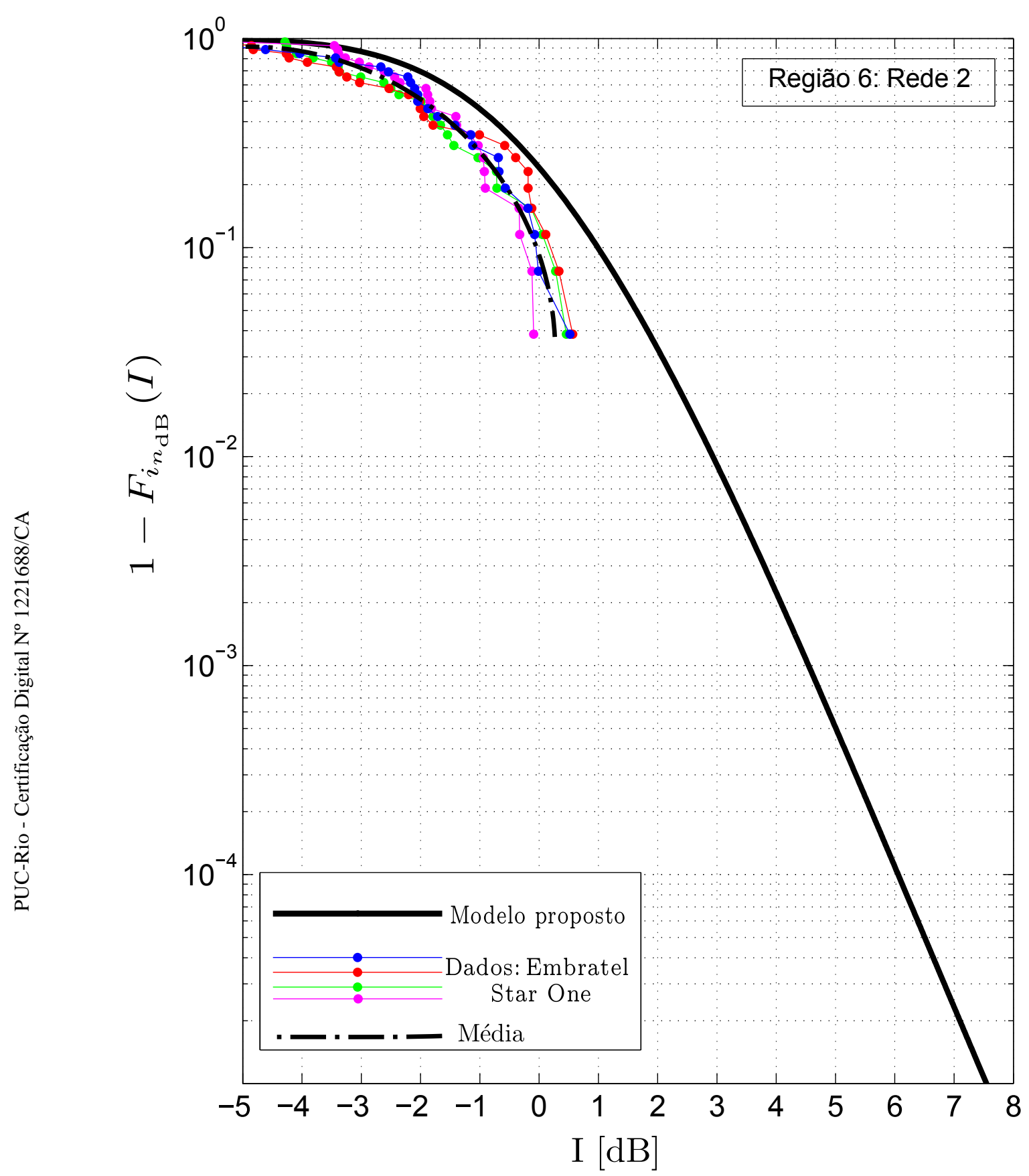

Figura 4.15: Função distribuição de probabilidade complementar correspondente à Rede 2, com 26 terminais VSAT na Região 6 . 


\subsubsection{3}

\section{Exemplo envolvendo a Região $\mathcal{R}_{4}$}

Este exemplo tem como objetivo ilustrar o comportamento do modelo proposto em situações nas quais os terminais VSAT podem operar com antenas de diferentes diâmetros, o que ocorre na região $\mathcal{R}_{4}$. Considera-se que a Rede 1 tem 11 terminais $\operatorname{VSAT}\left(\mathcal{N}_{T}=11\right)$ e a Rede 2 possui 9 terminais VSAT $\left(\mathcal{N}_{T}=9\right)$. Novamente, estes valores foram escolhidos de modo a possibilitar a comparação dos resultados obtidos com o modelo proposto com aqueles resultantes de dados reais fornecidos pela operadora de satélites Embratel Star One. Levando-se em conta que a área da Região $\mathcal{R}_{4}$ é de $725.217 \mathrm{~km}^{2}$, esses números de terminais conduzem aos valores de densidade de $1,516 \times 10^{-5}$ terminais por $\mathrm{km}^{2}$ e $1,241 \times 10^{-5}$ terminais por $\mathrm{km}^{2}$ para as redes 1 e 2 , respectivamente.

Com base nos comentários da Seção 4.1.2, considerou-se que, em ambas as redes, os terminais VSAT podem operar com dois possíveis tamanhos, equiprováveis, de antena $\left(N_{4}=2, d_{1}=1,2 \mathrm{~m}, d_{2}=1,8 \mathrm{~m}, P_{14}=0,5\right.$ e $\left.P_{24}=0,5\right)$. Para as densidades de e.i.r.p. transmitidas pelos terminais VSAT considerouse os valores apresentados na Tabela 4.2 , ou seja, $E_{14}=-1,11 \mathrm{~dB}(\mathrm{~W} / \mathrm{Hz})$ e $E_{24}=3,29 \mathrm{~dB}(\mathrm{~W} / \mathrm{Hz})$.

As figuras 4.16 e 4.17 ilustram, respectivamente, distribuições típicas (amostras de um PPP) de terminais VSAT das redes 1 e 2 na Região $\mathcal{R}_{4}$. Estas figuras incluem ainda a posição geográfica $R_{\text {ref }}$ utilizada no cálculo do valor de referência $i_{r e f}$, feito utilizando-se uma antena de diâmetro $d_{r e f}=1,2$ m. O comportamento estatístico (função distribuição de probabilidade complementar) da variável aleatória $i_{n_{\mathrm{dB}}}$, que caracteriza a densidade de potência interferente produzida pelos terminais VSAT da Rede 1 no enlace vítima, é apresentado na Figura 4.18 (linha cheia). A título de comparação, esta figura inclui ainda estimativas da função distribuição de probabilidade complementar de $i_{n_{\mathrm{dB}}}$, obtidas diretamente de dados reais fornecidos pela operadora de satélites, considerando-se quatro diferentes conjuntos de valores de erro de apontamento de antenas escolhidos aleatoriamente (pontos ligados por linhas). No caso de dados reais, esta figura inclui ainda a curva média (linha tracejada) obtida a partir de diferentes conjuntos de valores de erro de apontamento das antenas escolhidos aleatoriamente.

De maneira análoga, o comportamento estatístico da variável aleatória $i_{n_{\mathrm{dB}}}$, que caracteriza a densidade de potência interferente produzida pelos terminais VSAT da Rede 2 no enlace vítima, é apresentado na Figura 4.19 (linha cheia). Novamente, a título de comparação, esta figura inclui estimativas obtidas diretamente de dados reais fornecidos pela Embratel Star One, 


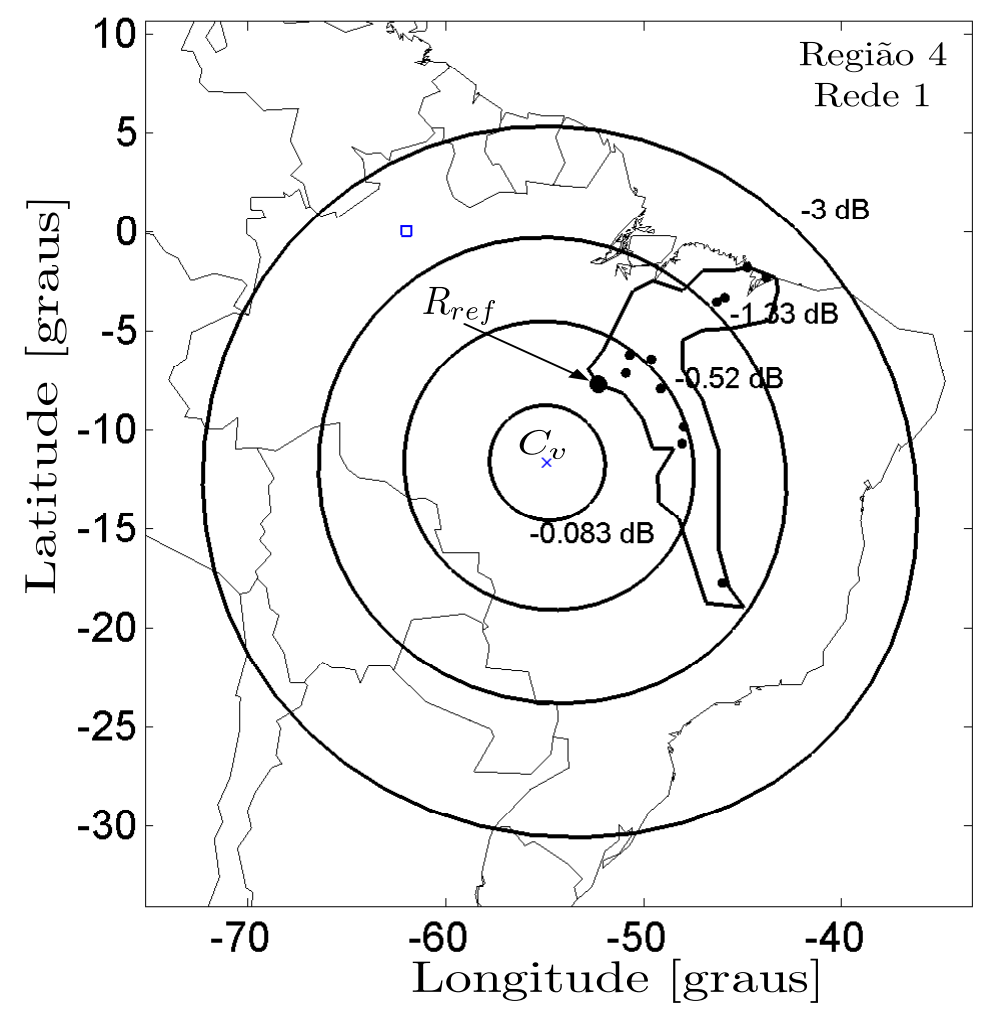

Figura 4.16: Amostra típica da distribuição de terminais da Rede 1 na Região 4 (PPP com densidade média de $1,516 \times 10^{-5}$ terminais por $\mathrm{km}^{2}$ ). Inclui a posição geográfica $R_{\text {ref }}$ utilizada no cálculo do valor de referência $i_{r e f}$ $\left(52,31^{\circ} \mathrm{W}, 7,76^{\circ} \mathrm{S}\right)$.

considerando-se quatro diferentes conjuntos de valores de erro de apontamento de antenas escolhidos aleatoriamente (pontos ligados por linhas). No caso de dados reais, esta figura também inclui ainda a curva média (linha tracejada) obtida a partir de diferentes conjuntos de valores de erro de apontamento das antenas escolhidos aleatoriamente.

Observe neste exemplo que em torno de $\mathrm{I}[\mathrm{dB}]=0$, existe uma diferença aceitável de aproximadamente 1,0 dB (rede 1) e 1,90 dB (rede 2), entre os resultados produzidos pelo modelo proposto e a curva média obtidos com base em dados reais, sendo o modelo proposto conservador em ambos os casos. O aumento desta diferença, em relação aos exemplos anteriores, se justifica pela incerteza adicional introduzida pela possibilidade de utilização equiprovável de antenas com dois diâmetros diferentes.

Observe que, no caso dos dados reais (ver curvas com pontos ligados por linhas nas figuras 4.18 e 4.19), a aleatoriedade esta relacionada com a escolha do terminal $T^{\prime}$ que vai transmitir em um determinado canal em um dado instante. E, considerou-se que os terminais VSAT têm a mesma probabilidade de ser escolhido. 




Figura 4.17: Amostra típica da distribuição de terminais da Rede 2 na Região 4 (PPP com densidade média de $1,241 \times 10^{-5}$ terminais por $\mathrm{km}^{2}$ ). Inclui a posição geográfica $R_{\text {ref }}$ utilizada no cálculo do valor de referência $i_{r e f}$ $\left(52,31^{\circ} \mathrm{W}, 7,76^{\circ} \mathrm{S}\right)$.

Novamente, por utilizar um modelo analítico na caracterização do comportamento estatístico do ângulo $\theta_{\mathbf{r}}$, o modelo proposto é capaz de fornecer a função distribuição de probabilidade de $i_{n_{\mathrm{dB}}}$ mesmo para valores bastante ínfimos de $P\left(i_{n_{\mathrm{dB}}}>I\right)$ (nas figuras estas probabilidade estão limitadas inferiormente por $10^{-5}$ ). Já as estimativas obtidas a partir de dados reais dependem da quantidade de dados considerados (basicamente do número de terminais da rede), conforme refletido nos resultados apresentados nas figuras 4.18 (11 terminais) e 4.19 (9 terminais). 


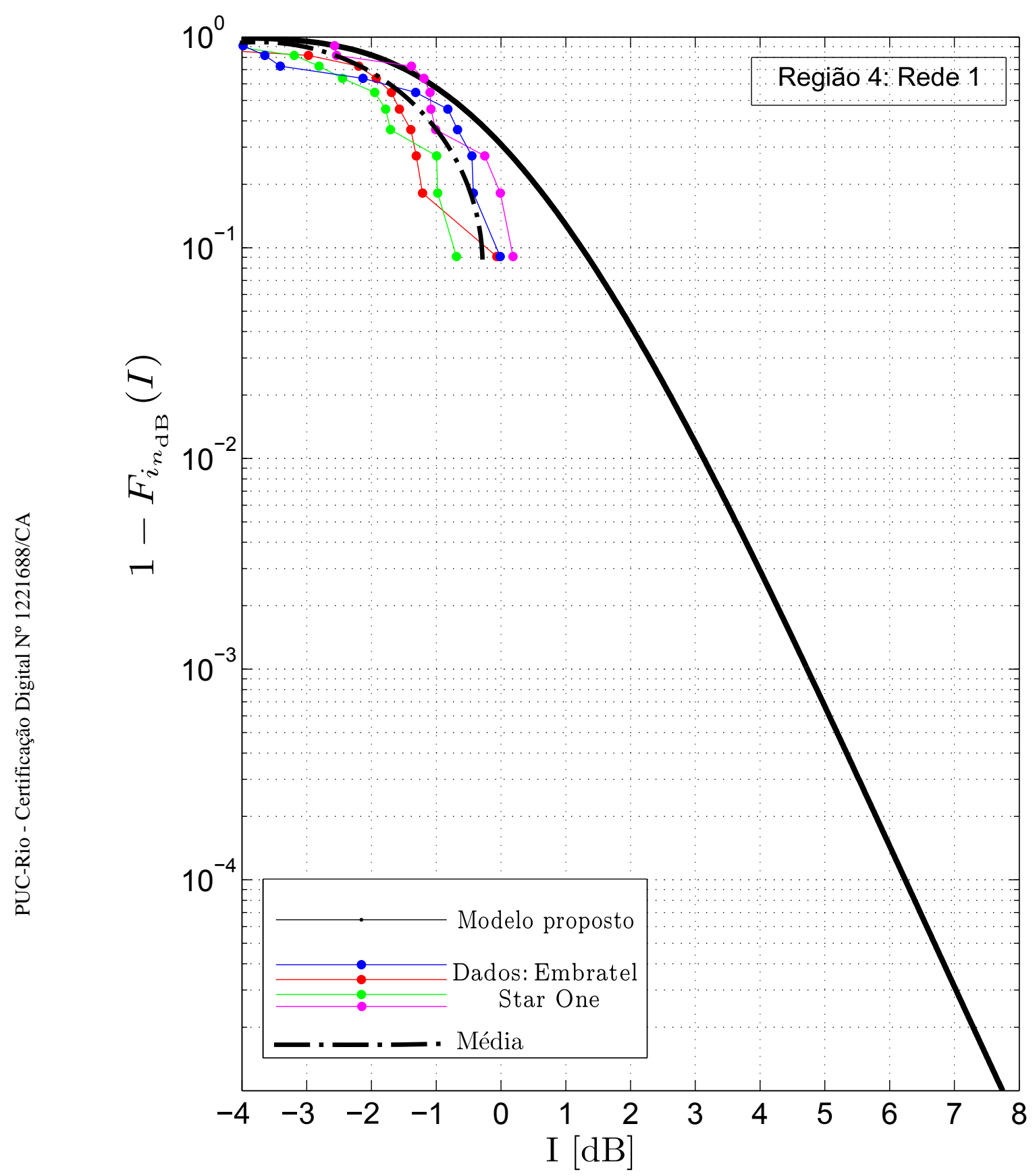

Figura 4.18: Função distribuição de probabilidade complementar correspondente à Rede 1, com 11 terminais VSAT na Região 4. 


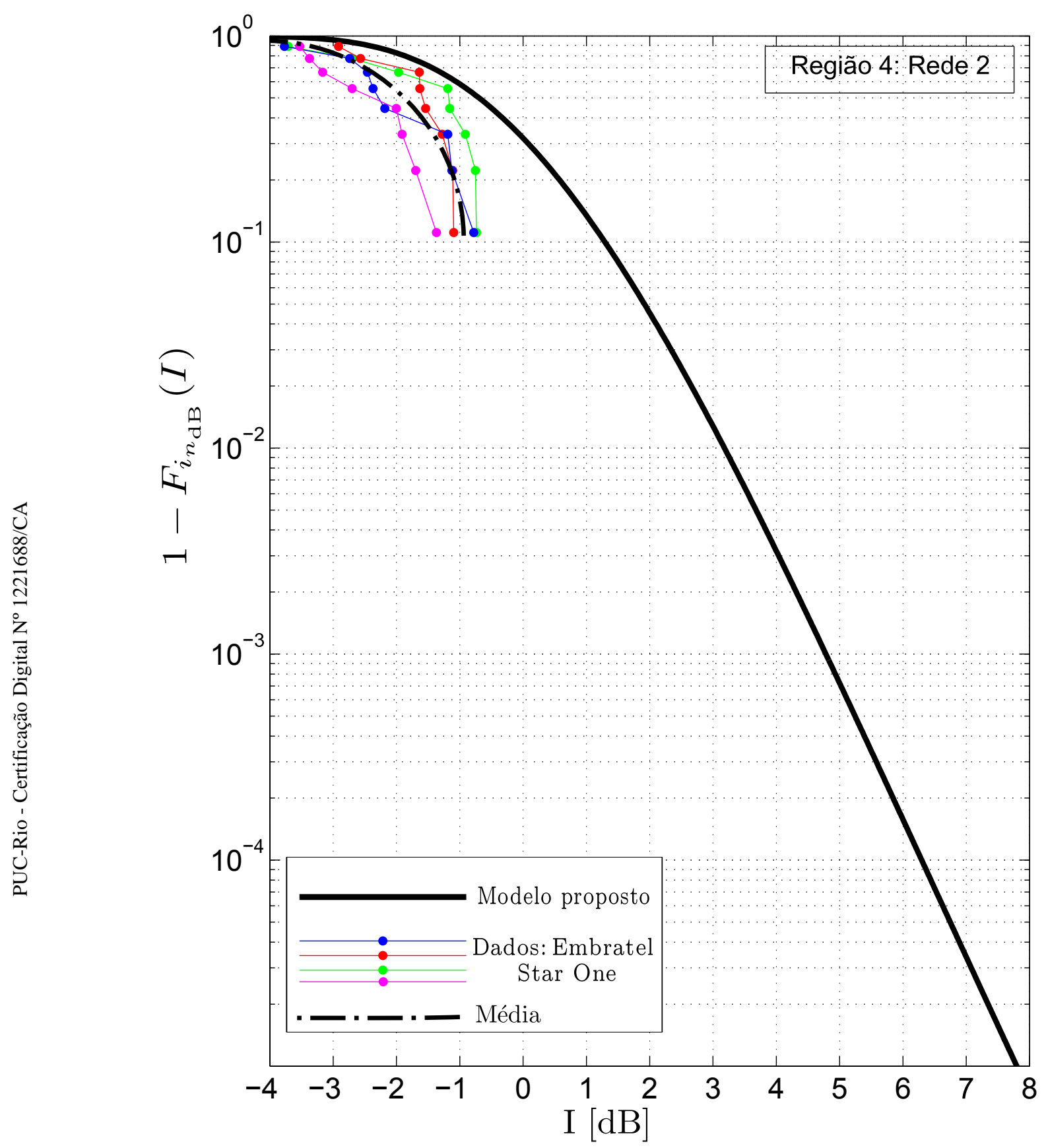

Figura 4.19: Função distribuição de probabilidade complementar correspondente à Rede 2, com 9 terminais VSAT na Região 4. 


\subsection{2}

\section{Regiões com múltiplas distribuições de terminais}

Nesta seção, são consideradas redes VSAT interferentes atuando numa área de serviço contendo múltiplos tipos de distribuição de terminais VSAT. Mais especificamente, são apresentados dois exemplos de redes VSAT operando em todo o território brasileiro. Conforme ressaltado na Seção 4.1, dois elementos importantes utilizados pelo modelo proposto são a distribuição demográfica e a distribuição da taxa de precipitação pluvial na área de serviço da rede, no caso em análise, do território brasileiro. Estes dados foram apresentados nas figuras 4.1 e 4.2 e utilizados na identificação de seis regiões no território brasileiro contendo uma densidade demográfica aproximadamente uniforme e, ao mesmo tempo, pouca variação nas características de precipitação. As regiões resultantes, foram ilustradas na Figura 4.3 e suas características apresentadas na Subseção 4.1.1.

Novamente, são considerados os enlaces (inbound) de duas redes VSAT: a primeira, aqui denominada Rede 1 , contendo 211 terminais $\operatorname{VSAT}\left(\mathcal{N}_{T}=211\right)$ e a segunda, Rede 2 , contendo 78 terminais $\operatorname{VSAT}\left(\mathcal{N}_{T}=78\right)$. Estes valores foram escolhidos de modo a possibilitar a comparação dos resultados obtidos com o modelo proposto com aqueles resultantes de dados reais, correspondentes a duas redes VSAT, fornecidos pela operadora de satélites Embratel Star One.

Conforme indicado na Tabela 4.1 da Subseção 4.1.2, o modelo proposto considera que os terminais VSAT podem operar com antenas de três diâmetros diferentes $0,96,1,20$ e 1,80 metros. As densidades de e.i.r.p. transmitidas pelos terminais VSAT foram determinadas de acordo com o procedimento apresentado na Subseção 4.1.3, do qual resultaram os valores apresentados na Tabela 4.2, ou seja, $-3,42,-1,11$, e $3,29 \mathrm{~dB}(\mathrm{~W} / \mathrm{Hz})$ correspondentes respectivamente às antenas de diâmetros 0,96, 1,20 e 1,80 metros. Os valores de densidade de e.i.r.p. utilizados em cada uma das regiões identificadas são aqueles apresentados na Tabela 4.3 da Subseção 4.1.3.

Em cada uma das regiões, as posições geográficas dos terminais VSAT foram modeladas por processos pontuais bidimensionais de Poisson (PPP). O número médio $\mathcal{N}_{m}$ e a densidade $\lambda_{m}$ de terminais VSAT em cada uma das regiões foram determinados utilizando-se (4-6) e (4-8) e são apresentados nas tabelas 4.7 e 4.8 para as redes 1 e 2 , respectivamente.

As figuras 4.20 e 4.21 ilustram, respectivamente, distribuições típicas de terminais VSAT, produzidas pelo modelo proposto para as redes 1 e 2 no território brasileiro. Estas figuras incluem ainda o centro do feixe da antena receptora $C_{v}$ do satélite vítima $S$ e a posição geográfica $R_{r e f}\left(54,90^{\circ} \mathrm{W}, 9,70^{\circ} \mathrm{S}\right)$ utilizada no cálculo do valor de referência $i_{r e f}$, em ambas redes. O valor de 
Tabela 4.7: Número $\mathcal{N}_{m}$ e densidade $\lambda_{m}$ de terminais de terminais por região (Rede $1-\mathcal{N}_{T}=211$ ).

\begin{tabular}{|c|c|c|}
\hline \hline Região & $\mathcal{N}_{m}$ & $\lambda_{m}, m=1, \ldots, 6\left[\right.$ terminais $\left./ \mathrm{km}^{2}\right]$ \\
\hline $\mathcal{R}_{1}$ & 84 & $1,127 \times 10^{-4}$ \\
\hline $\mathcal{R}_{2}$ & 7 & $5,809 \times 10^{-6}$ \\
\hline $\mathcal{R}_{3}$ & 65 & $1,338 \times 10^{-4}$ \\
\hline $\mathcal{R}_{4}$ & 9 & $1,248 \times 10^{-5}$ \\
\hline $\mathcal{R}_{5}$ & 10 & $2,917 \times 10^{-5}$ \\
\hline $\mathcal{R}_{6}$ & 36 & $7,054 \times 10^{-6}$ \\
\hline \hline
\end{tabular}

Tabela 4.8: Número $\mathcal{N}_{m}$ e densidade $\lambda_{m}$ de terminais de terminais por região (Rede $2-\mathcal{N}_{T}=78$ ).

\begin{tabular}{|c|c|c|}
\hline \hline Região & $\mathcal{N}_{m}$ & $\lambda_{m}, m=1, \ldots, 6\left[\right.$ terminais $\left./ \mathrm{km}^{2}\right]$ \\
\hline $\mathcal{R}_{1}$ & 31 & $4,167 \times 10^{-5}$ \\
\hline $\mathcal{R}_{2}$ & 3 & $2,147 \times 10^{-6}$ \\
\hline $\mathcal{R}_{3}$ & 24 & $4.946 \times 10^{-5}$ \\
\hline $\mathcal{R}_{4}$ & 3 & $4,614 \times 10^{-6}$ \\
\hline $\mathcal{R}_{5}$ & 4 & $1,078 \times 10^{-5}$ \\
\hline $\mathcal{R}_{6}$ & 13 & $2,607 \times 10^{-6}$ \\
\hline \hline
\end{tabular}

$i_{\text {ref }}$, foi determinado por meio de (4-2), obtendo-se $i_{\text {ref }}=-202.17 \mathrm{~dB}(\mathrm{~W} / \mathrm{Hz})$, correspondente a um terminal VSAT com antena transmissora de 0,96 metros de diâmetro. 


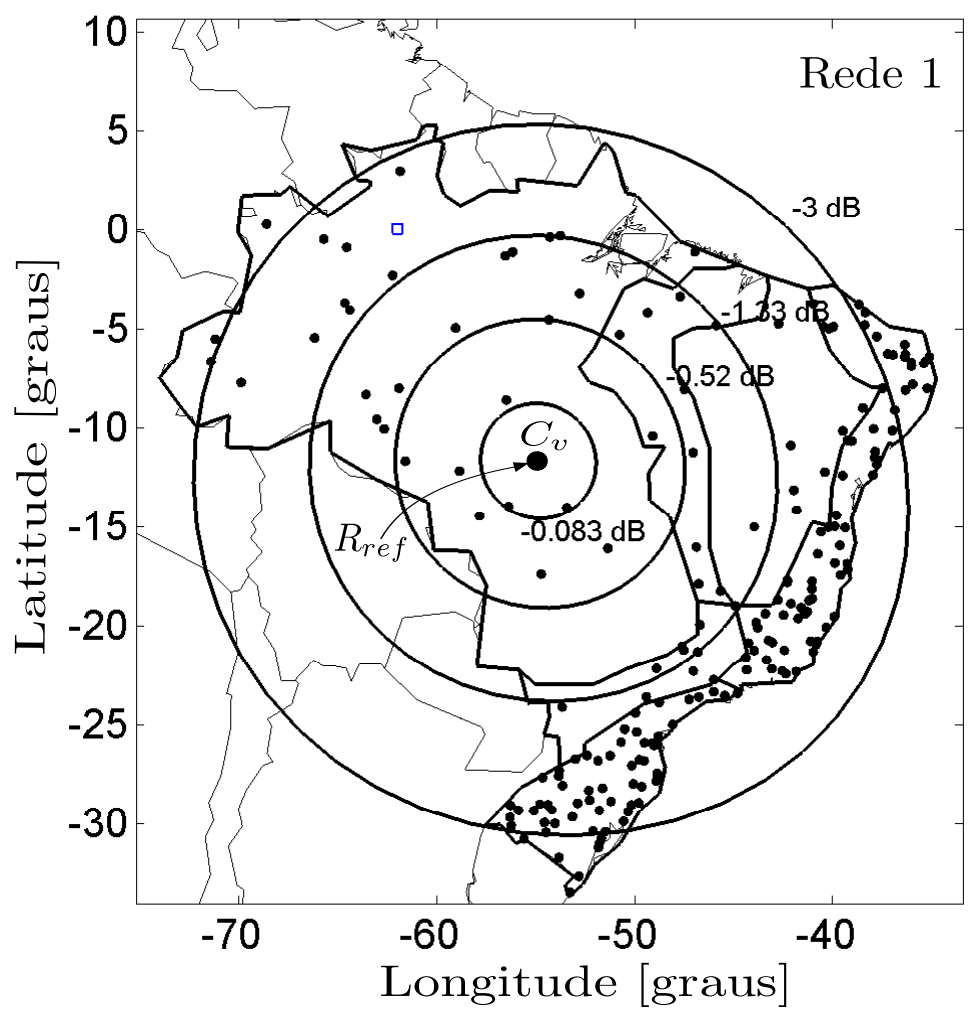

Figura 4.20: Amostras típicas das distribuições de terminais correspondentes a cada região da Rede 1 no território brasileiro (PPP com densidades médias por região [terminais $/ \mathrm{km}^{2}$ ], apresentada na Tabela 4.7$)$, com $R_{\text {ref }}$ $\left(54,90^{\circ} \mathrm{W}, 9,70^{\circ} \mathrm{S}\right)$. 


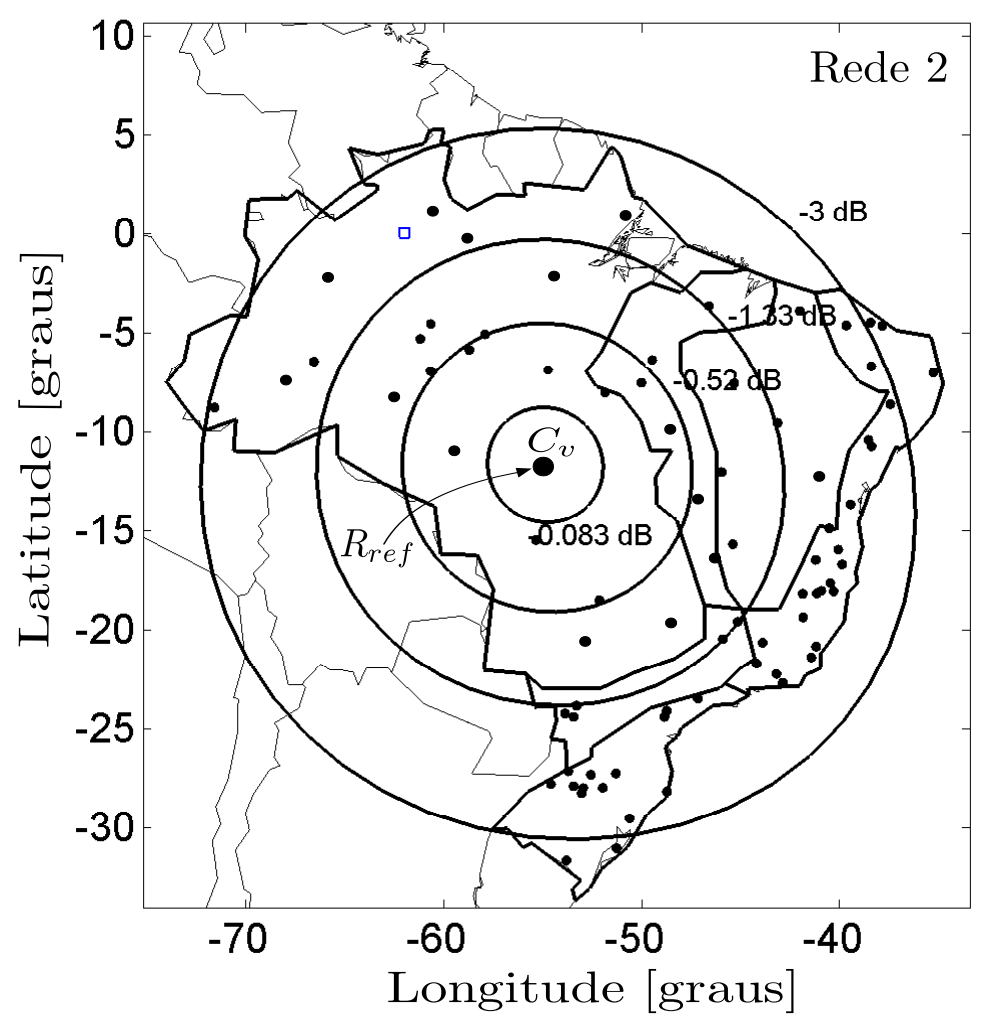

Figura 4.21: Amostras típicas das distribuições de terminais correspondentes a cada região da Rede 2 no território brasileiro (PPP com densidade média por região [terminais $/ \mathrm{km}^{2}$ ], apresentada na Tabela 4.8 ), com $R_{\text {ref }}$ $\left(54,90^{\circ} \mathrm{W}, 9,70^{\circ} \mathrm{S}\right)$. 
O comportamento estatístico (função distribuição de probabilidade complementar) da variável aleatória $i_{n_{\mathrm{dB}}}$, que caracteriza a densidade de potência interferente produzida pelos terminais VSAT no enlace vítima, foi obtido a partir de (3-53) e (3-52).

Inicialmente foram determinadas, para cada uma das regiões consideradas, as funções distribuição de probabilidade condicionais em (3-55). Essas funções distribuição de probabilidade são apresentadas nas figuras 4.22 e 4.23, respectivamente para as redes interferentes 1 e 2 (uma comparação destas curvas com aquelas obtidas a partir de dados reais é apresentado no Apêndice F). Em seguida, a função distribuição de probabilidade complementar da densidade de potência interferente normalizada $i_{n_{\mathrm{dB}}}$ devida a todos os $\mathcal{N}_{T}$ terminais VSAT (toda a área de serviço) foi determinada utilizando-se (3-54). Os valores das probabilidades $P\left(\mathbf{r} \in \mathcal{R}_{m}\right)$ que aparecem em (3-54) foram obtidos a partir de (4-9) e, no caso das redes $1\left(\mathcal{N}_{T}=211\right.$ terminais $)$ e $2\left(\mathcal{N}_{T}=78\right.$ terminais $)$, são apresentados na Tabela 4.9.

Tabela 4.9: Valores de probabilidade $P\left(\mathbf{r} \in \mathcal{R}_{m}\right)$ de cada região $\mathcal{R}_{m}(m=$ $1, \ldots, 6)$, para a Rede 1 e Rede 2 .

\begin{tabular}{|c|c|c|}
\hline \hline Região & $P\left(\mathbf{r} \in \mathcal{R}_{m}\right)$ Rede 1 & $P\left(\mathbf{r} \in \mathcal{R}_{m}\right)$ Rede 2 \\
\hline $\mathcal{R}_{1}$ & 0,39810 & 0,39744 \\
\hline $\mathcal{R}_{2}$ & 0,03318 & 0,03846 \\
\hline $\mathcal{R}_{3}$ & 0,30806 & 0,30769 \\
\hline $\mathcal{R}_{4}$ & 0,04265 & 0,03846 \\
\hline $\mathcal{R}_{5}$ & 0,04739 & 0,05128 \\
\hline $\mathcal{R}_{6}$ & 0,17062 & 0,16667 \\
\hline \hline
\end{tabular}




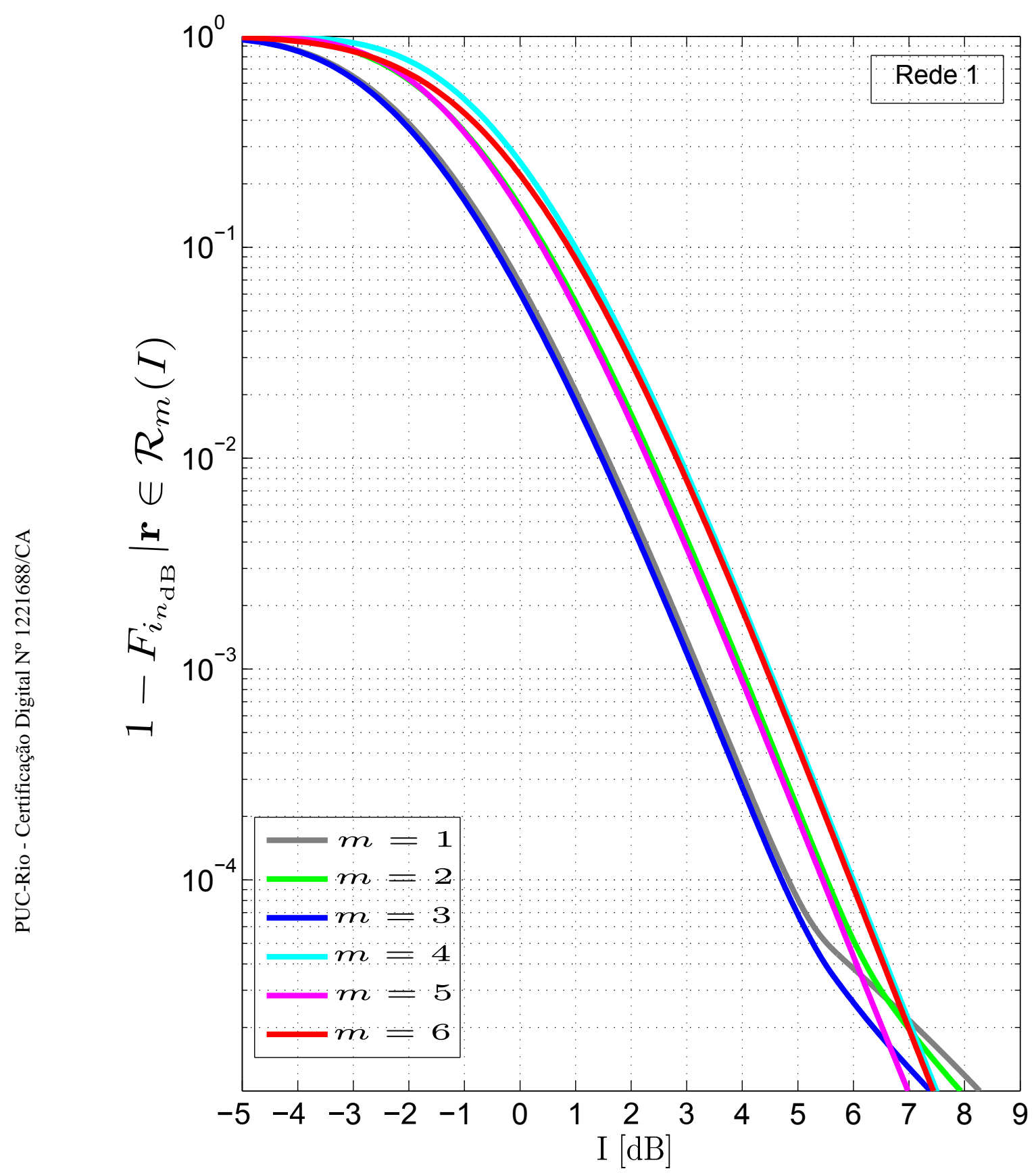

Figura 4.22: Função distribuição de probabilidade complementar correspondente à região $\mathcal{R}_{1}, \mathcal{R}_{2}, \mathcal{R}_{3}, \mathcal{R}_{4}, \mathcal{R}_{5}$, e $\mathcal{R}_{6}$ da Rede 1 , com $R_{\text {ref }}$ $\left(54,90^{\circ} \mathrm{W}, 9,70^{\circ} \mathrm{S}\right)$. 


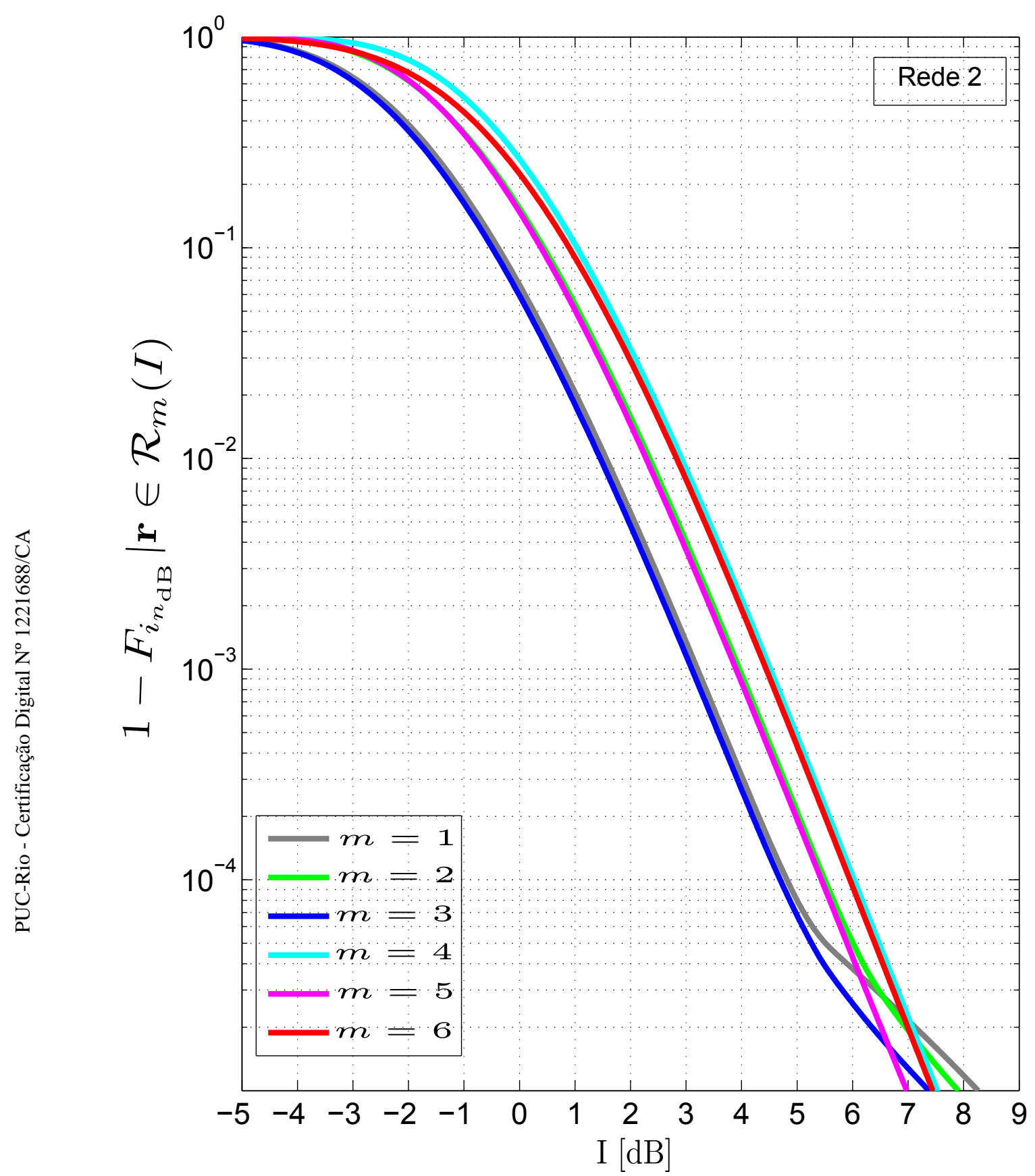

Figura 4.23: Função distribuição de probabilidade complementar correspondente à região $\mathcal{R}_{1}, \mathcal{R}_{2}, \mathcal{R}_{3}, \mathcal{R}_{4}, \mathcal{R}_{5}$, e $\mathcal{R}_{6}$ da Rede 2 , com $R_{\text {ref }}$ $\left(54,90^{\circ} \mathrm{W}, 9,70^{\circ} \mathrm{S}\right)$. 
O resultado obtido para o comportamento estatístico da densidade de potência interferente normalizada $i_{n_{\mathrm{dB}}}$ devida a todos os 211 terminais VSAT (toda a área de serviço) da Rede 1 é apresentado na Figura 4.24 (linha cheia). A título de comparação, esta figura inclui as estimativas obtidas diretamente de dados reais (211 terminais), fornecidos pela operadora de satélites Embratel Star One, considerando-se quatro diferentes conjuntos de valores de erro de apontamento de antenas escolhidos aleatoriamente (pontos ligados por linhas). No caso de dados reais, esta figura inclui ainda a curva média (linha tracejada) obtida a partir de diferentes conjuntos de valores de erro de apontamento das antenas escolhidos aleatoriamente.

De maneira análoga, o resultado obtido para o comportamento estatístico da densidade de potência interferente normalizada $i_{n_{\mathrm{dB}}}$ devida a todos os 78 terminais VSAT (toda a área de serviço) da Rede 2 é apresentado na Figura 4.25 (linha cheia). Novamente, a título de comparação, esta figura inclui as estimativas obtidas diretamente de dados reais (78 terminais), fornecidos pela operadora de satélites Embratel Star One, considerando-se quatro diferentes conjuntos de valores de erro de apontamento de antenas escolhidos aleatoriamente (pontos ligados por linhas). No caso de dados reais, esta figura também inclui ainda a curva média (linha tracejada) obtida a partir de diferentes conjuntos de valores de erro de apontamento das antenas escolhidos aleatoriamente.

Observe neste exemplo que em torno de $\mathrm{I}[\mathrm{dB}]=0$, no caso da Rede 1 existe uma concordância bastante grande entre os resultados produzidos pelo modelo proposto e a curva média obtidos com base em dados reais.

Já no caso da Rede 2, existe uma diferença aceitável de aproximadamente $0,34 \mathrm{~dB}$, entre os resultados produzidos pelo modelo proposto e a curva média obtidos com base em dados reais, sendo o modelo proposto conservador em ambos os casos.

Observe nas figuras 4.24 e 4.25, que a aleatoriedade envolvendo regiões com diferentes caraterísticas de distribuição de terminais depende da probabilidade $P\left(\mathbf{r} \in \mathcal{R}_{m}\right)$ do canal TDMA estar sendo utilizado por um terminal $T^{\prime}$ da região $\mathcal{R}_{m}$ para $m=1, \ldots, M$, estimadas por (4-9)

Note ainda que, em ambos os casos, conforme esperado, por utilizar um modelo analítico na caracterização do comportamento estatístico do ângulo $\theta_{\mathbf{r}}$, o modelo proposto é capaz de fornecer valores da a função distribuição de probabilidade complementar de $i_{n_{\mathrm{dB}}}$ mesmo para valores bastante ínfimos de $P\left(i_{n_{\mathrm{dB}}}>I\right)$ (nas figuras estas probabilidade estão limitadas inferiormente por $10^{-5}$ ). Já as estimativas obtidas a partir de dados reais dependem da quantidade de dados considerados (basicamente do número de terminais da 
rede), conforme refletido nos resultados apresentados nas figuras 4.24 (211 terminais) e 4.25 (78 terminais).

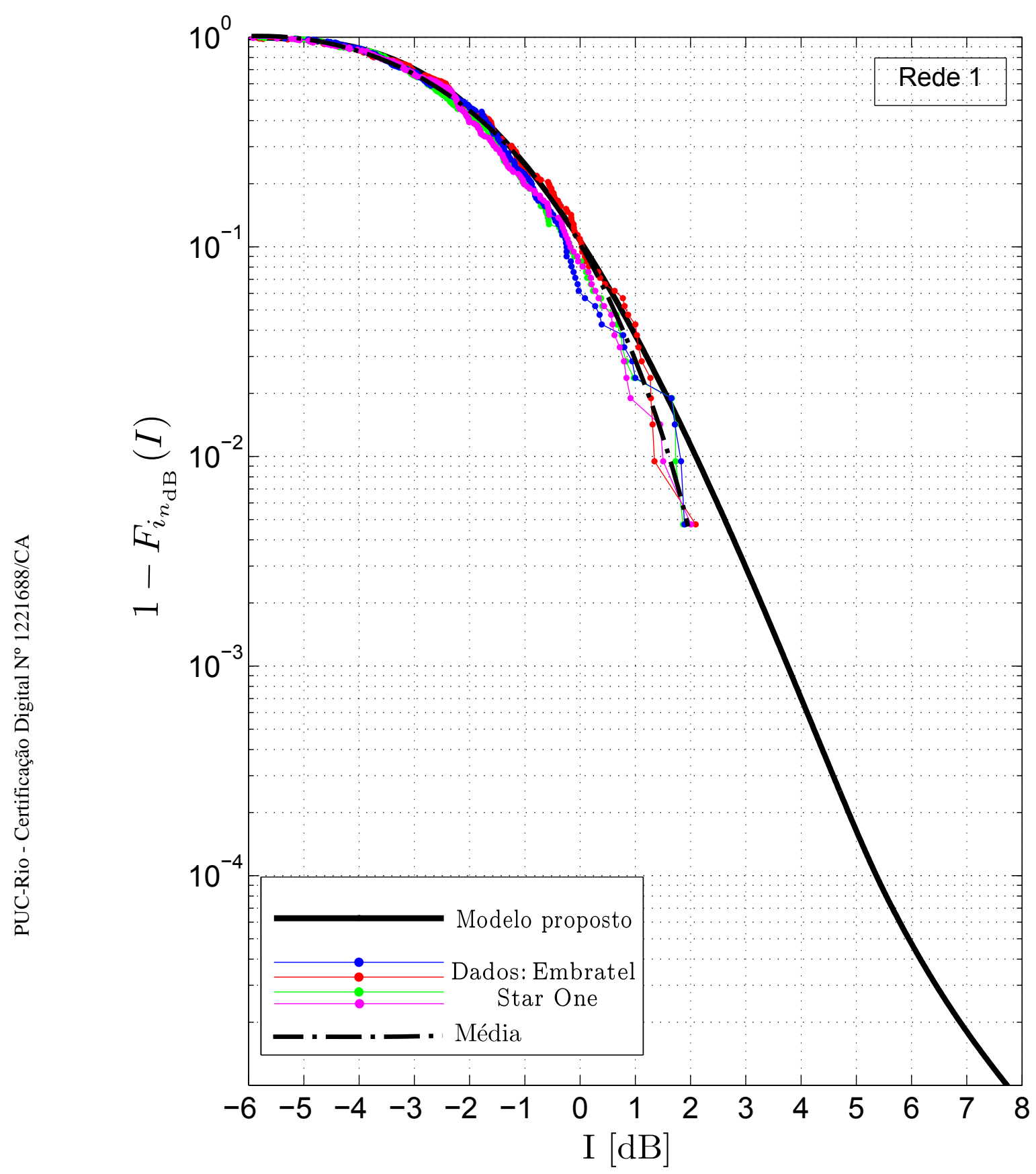

Figura 4.24: Função distribuição de probabilidade complementar correspondente à Rede 1 , com 211 terminais VSAT, com $R_{\text {ref }}\left(54,90^{\circ} \mathrm{W}, 9,70^{\circ} \mathrm{S}\right)$. 




Figura 4.25: Função distribuição de probabilidade complementar correspondente à Rede 2 , com 78 terminais VSAT, com $R_{\text {ref }}\left(54,90^{\circ} \mathrm{W}, 9,70^{\circ} \mathrm{S}\right)$. 


\section{Conclusões e sugestões para trabalhos futuros.}

\section{1 \\ Conclusão}

Neste trabalho foi apresentado um modelo matemático que permite avaliar o comportamento estatístico da potência interferente produzida por redes VSAT que utilizam o protocolo MF-TDMA de múltiplo acesso por divisão no tempo e frequência. O desenvolvimento analítico do modelo visou considerar principalmente a dependência entre as posições geográficas das estações terrenas transmissoras interferentes, e a densidade de e.i.r.p. na direção de máxima radiação das antenas transmissoras. Além disso foram também considerados os erros de apontamento das antenas das estações terrenas transmissoras.

A aplicação do modelo requer, apenas, o conhecimento do número de terminais da rede e de informações sobre a distribuição demográfica e a distribuição da taxa de precipitação ao longo da área de serviço da rede. Com base nestes dados, foram determinados os possíveis diâmetros das antenas transmissoras e a densidade de e.i.r.p. transmitida por cada uma delas. As posições geográficas das estações terrenas foram modeladas por um processo pontual bi-dimensional adequado (e.g. processo pontual de Poisson).

Um aspecto importante do modelo proposto diz respeito à sua aplicabilidade a redes VSAT/MF-TDMA com áreas de serviço envolvendo diferentes tipos de distribuição de terminais.

O modelo proposto foi aplicado a quatro cenários onde as transmissões dos terminais VSAT de uma determinada rede atingem o receptor de um satélite vizinho (a três graus de separação orbital). Os resultados produzidos pelo modelo proposto foram comparados àqueles obtidos a partir de dados reais (posição geográfica dos terminais, diâmetros de suas antenas e suas potências de transmissão) de duas redes, contendo respectivamente 211 e 78 terminais, fornecidos pela operadora de satélites Embratel StarOne.

Os três primeiros cenários envolveram exemplos de redes VSAT com áreas de serviço contendo um único tipo de distribuição de terminais. Mais especificamente, três situações foram abordadas: alta densidade de terminais com um único diâmetro de antena transmissora, baixa densidade de terminais com um único diâmetro de antena transmissora e baixa densidade de terminais com diferentes diâmetros de antena transmissora. Conforme indicado na Subseção 
4.2.1, no primeiro exemplo houve uma boa concordância, e no segundo e terceiro exemplos houve uma aceitável concordância entre os resultados produzidos pelo modelo e aqueles obtidos a partir de dados reais.

O primeiro exemplo mostrou, para as duas redes consideradas que em torno de $\mathrm{I}[\mathrm{dB}]=0$, existe uma concordância bastante grande entre os resultados produzidos pelo modelo proposto e a curva média obtidos com base em dados reais. No segundo exemplo, observou-se que em torno de $\mathrm{I}[\mathrm{dB}]=0$, existe uma diferença aceitável de aproximadamente $0,85 \mathrm{~dB}$ (rede 1) e 0,70 $\mathrm{dB}$ (rede 2), entre os resultados produzidos pelo modelo proposto e a curva média obtidos com base em dados reais, sendo o modelo proposto conservador em ambos os casos. No terceiro exemplo, observou-se que em torno de $\mathrm{I}[\mathrm{dB}]=0$, existe uma diferença aceitável de aproximadamente 1,0 dB (rede 1) e 1,90 dB (rede 2), entre os resultados produzidos pelo modelo proposto e a curva média obtidos com base em dados reais, sendo o modelo proposto conservador em ambos os casos devida à incerteza adicional introduzida pela utilização equiprovável de antenas com dois diâmetros diferentes.

O modelo proposto foi também aplicado a um cenário envolvendo redes VSAT/MF-TDMA com área de serviço abrangendo todo o território brasileiro, o que reflete uma situação onde, dependendo da região considerada, diferentes tipos de distribuição de terminais são encontrados. Conforme mostrado na Subseção 4.2.2, os resultados obtidos neste caso mais complexo foram bem próximos daqueles obtidos a partir de dados reais, para as duas redes consideradas. Na Rede 1 observou-se uma concordância bastante grande, na Rede 2 houve uma diferença de aproximadamente $0,34 \mathrm{~dB}$, em torno ao valor de referência $\mathrm{I}[\mathrm{dB}]=0$ correspondente à estimativa média obtida a partir de dados reais fornecidos pela operadora de satélites Embratel Star One, considerandose os resultados como aceitáveis e conservadores, de aqueles obtidos a partir de dados reais.

Em resumo, os resultados apresentados indicam que o modelo analítico proposto é robusto e possui um ótimo desempenho em condições diversas de distribuição de terminais que utilizam antenas de diferentes diâmetros e operam com diferentes potências de transmissão. 


\section{2}

\section{Sugestões para trabalhos futuros.}

Conforme ressaltado no Capítulo 3, o modelo matemático proposto é aplicável a redes VSAT com outros tipos de distribuição de terminais como, por exemplo, distribuições com clusters. Estas distribuições podem, por exemplo, ser modeladas por processos estocásticos pontuais de Cox ou de Neyman-Scott. O primeiro generaliza processo de Poisson considerando que a medida de intensidade é aleatória, e o segundo produz um clustering independente e homogêneo a partir de Poisson homogêneo. Esses dois processos são considerados, em termos gerais, como processos pontuais com graus mais altos de variabilidade que os processos pontuais Binomial e Poisson. Trabalhos futuros podem incluir, por exemplo, uma avaliação do modelo proposto quando aplicado a redes com distribuição de terminais em clusters. A dificuldade prevista nesta avaliação seria a identificação de situações reais com este tipo de distribuição e a obtenção, dos operadores de satélite, de informações sobre estas redes. 


\section{Referências bibliográficas}

[1] ITU-R Statistical methodology to assess time-varying interference produced by a geostationary fixed-satellite service network of earth stations operating with MF-TDMA schemes to geostationary fixed-satellite service network. Recommendation ITU-R S.2029, Geneva,2012.

[2] ITU-R Working Document: Methodology to estimate the sensitivity of GSO FSS interference levels to the geographical location of earth stations communicating with satellites in the fixed-satellite service in the $14 \mathrm{GHz}$ and 29.5-30 GHz frequency bands. Document 4A/316-E. Radiocommunication Study Groups, Geneva, 26 September 2013.

[3] ITU-R Working Document: Methodology to estimate the sensitivity of GSO FSS interference levels to the geographical location of earth stations communicating with satellites in the fixed-satellite service in the $14 \mathrm{GHz}$ and 29.5-30 GHz frequency bands. Annex 22 to Document 4A/343-E. Radiocommunication Study Groups, Geneva, 17 October 2013.

[4] ITU-R Preliminary Draft Report: Methodology to estimate the sensitivity of GSO FSS interference levels to the geographical location of earth stations communicating with satellites in the fixed-satellite service in the $14 \mathrm{GHz}$ and 29.5-30 GHz frequency bands. Annex 3 to Document 4A/468E. Radiocommunication Study Groups, Geneva, 19 February 2014.

[5] ITU-R Preliminary Draft Report: Methodology to estimate the sensitivity of GSO FSS interference levels to the geographical location of earth stations communicating with satellites in the fixed-satellite service in the $14 \mathrm{GHz}$ and 29.5-30 GHz frequency bands. Annex 21 to Document 4A/591-E. Radiocommunication Study Groups, Geneva, 23 July 2014.

[6] ITU-R Report: Methodology to estimate the sensitivity of GSO FSS interference levels to the geographical location of earth stations communicating with satellites in the fixed-satellite service in the $14 \mathrm{GHz}$ and $30 \mathrm{GHz}$ frequency ranges. Report ITU-R S.2362-0, Geneva, June 2015.

[7] Serguei Primak, Valeri Kontorovich, Vladimir Lyandres Stochastic Methods and Their Applications to Communications. First Edition, Cap. 2, e 8. John Wiley \& Sons Ltd, 2004. 
[8] Andreas F. Molisch Wireless Communications. Second Edition, Cap. 5, e 12. John Wiley \& Sons Ltd, 2011.

[9] Sung Nok Chiu, Dietrich Stoyan, Wilfrid S. Kendallm, Joseph Mecke Stochastic Geometry and its Applications. Third edition, Cap. 1, 2, 4, e 5, 2013 .

[10] ITU-R Maximum permissible levels of interference in a satellite network (GSO/FSS; non-GSO/FSS; non-GSO/MSS feeder links) in the fixedsatellite service caused by other codirectional FSS networks below $30 \mathrm{GHz}$. Recomendação ITU-R S.1323, Geneva, 2002.

[11] ITU-R Maximum permissible levels of off-axis e.i.r.p. density from earth stations in geostationary-satellite orbit networks operating in the fixedsatellite service transmitting in the $6 \mathrm{GHz}, 13 \mathrm{GHz}, 14 \mathrm{GHz}$ and $30 \mathrm{GHz}$ frequency bands. Recomendação ITU-R S.524-9, Geneva, 2006.

[12] ITU-R Maximum permissible levels of off-axis e.i.r.p. density from very small aperture terminals (VSAT's). Recomendação ITU-R S.7281, Geneva, 2006.

[13] ITU-R Mathematical model of average and related radiation patterns for line-of-sight point-to-point fixed wireless system antennas for use in certain coodination studies and interference assessment in the frequency range from $1 \mathrm{GHz}$ to about $70 \mathrm{GHz}$. Recomendação ITU-R F.1245-2, Geneva, 2012.

[14] ITU-R Satellite antenna radiation pattern for use as a design objective in the fixed-satellite service employing geostationary satellites. Rec. ITU-R S.672-4, Geneva, 1997.

[15] ITU-R Characteristics of precipitation for propagation modelling. Rec.ITU-R P.837-6, Geneva, 2012.

[16] Américo A. Rubin de Celis Vidal and José Mauro P. Fortes, Statistical behaviour of the interference produced by MF TDMA VSAT networks. Conference of Computational Interdisciplinary Science (CCIS 2016), São Jose dos Campos-SP, november 2016. 
[17] Alberth Tamo and José Mauro P. Fortes, Satellite interference evaluation: joint effect of modeling earth station locations and earth station antenna sidelobe gains as random variables. International Journal of Satellite communications and networking, Jonh Wiley, August 2014.

[18] Instituto Brasileiro de Geográfica e Estatística, Mapas Temáticos: densidade demografica do Brasil. http://mapas.ibge.gov.br/tematicos/ demografia, 2010.

[19] Instituto Brasileiro de Geográfica e Estatística, Estimativas populacionais para os municípios e para as Unidades da Federação brasileiros - julio de 2015. http://www.ibge.gov.br/home/estatistica/populacao/ estimativa2015/default.shtm, 2015.

[20] José Paulo Almeida, Jose Mauro Pedro Fortesm Weiler Alves Finamore, Probabilidade, Variáveis Aleatórias e Processos Estocásticos. Editora PUC-Rio,2008 .

[21] Rodney Josué Biezuner, Notas de Aula: Medida e Integração. Departamento de Matemática, Instituto de Ciências Exatas (ICEx) Universidade Federal de Minas Gerais (UFMG),março de 2012 .

[22] F. Baccelli, B. Blaszczyszyn, and P. Muhlethaler, Stochastic Analysis of Spatial and Opportunistic ALOHA. IEEE J. Sel. Areas Commun., vol. 27, no. 7, September 2009.

[23] S. Srinivasa and M. Haenggi, Distance Distributions in Finite Uniformly Random Networks: Theory and Applications. IEEE Trans. Veh. Technol., vol. 59, February 2010.

[24] William Rucklidge Efficient Visual Recognition Using the Hausdorff Distance. Springer,1996.

[25] Kingman, J. F. C., Poisson Processes. Oxford Studies in Probability, Oxford University Press, 1993.

[26] Lewis, P. A. W. and Shedler, G. S., Simulation of non-homogeneous Poisson processes by thinning. Naval Res. Logist. Quart., 1979.

[27] Matthes, K., Kerstan, J., and Mecke, J., In

nitely Divisible Point Processes. John Wiley and Sons Ltd, Chichester, 1978. 
[28] Kallenberg, O. ,Foundations of Modern Probability. Springer-Verlag, New York, 2nd edition, 2002.

[29] Daley, D. J. and Vere-Jones, D., An Introduction to the Theory of Point Processes. Volume II: General Theory and Structure. Springer-Verlag, New York, 2nd edition, 2008.

[30] M. Souryal, B. Vojcic and R. Pickholtz Ad hoc, Multihop CDMA Networks with Route Diversity in a Rayleigh Fading Channel. in Proc. IEEE Military Commun. Conf.(MILCOM01), October 2001.

[31] Van Lieshout, M. N. M. and Baddeley, A. J., A non-parametric measure of spatial interaction in point patterns, Statist. Neerl. 1996.

[32] Coles, P. and Jones, B., A lognormal model for the cosmological mass distribution. Not. R. Astr. Soc., 1991.

[33] Moller, J. and Waagepetersen, R. P., Statistical Inference and Simulation for Spatial Point Processes. Chapman and Hall/CRC, Boca Raton, 2004.

[34] Stoyan, D. and Stoyan, H., On one of Matérn's hard-core point process models. Math. Nachr. 122, 1985.

[35] Stoyan, D. and Schlather, M., Random sequential adsorption: relationship to dead leaves and characterization of variability. ,J. Stat. Phys. 100, 2000. 


\section{A \\ Geometria Estocástica}

Em Geometria Estocástica os Processos Pontuais (Point Process), conhecidos também como padrões de pontos aleatórios (random point patterns) desempenham um papel fundamental. Em muitos problemas de geometria estocástica padrões de objetos geométricos são interpretados como um sistema de pontos em um espaço representativo. Um ponto neste sistema representa um objeto geométrico particular no padrão original. Para estabelecer a notação utilizada na caracterização dos processos pontuais e seus modelos, uma revisão dos conceitos básicos da Geometria Estocástica é apresentada neste Apêndice.

Neste Apêndice, é feita a abordagem do processo pontual mais elementar, conhecido como Processo Pontual Binomial (Binomial point process), e do mais importante padrão de pontos aleatórios, denominado Processo Pontual de Poisson (Poisson Point Process) utilizado como base para a construção de outros modelos mais complexos. Um dado padrão de pontos aleatórios pode exibir vários tipos de interações entre seus pontos constituintes. Assim por exemplo, os pontos do processo podem ocorrer em grupos (clusters) ou podem apresentar uma distância mínima entre os pontos (hard-core distance).

Conceitos apresentados neste Apêndice são amplamente conhecidos e podem ser encontrados com maior detalhe por exemplo, em [9].

\section{A.1}

\section{Processo pontual Binomial}

O mais elementar exemplo de um processo pontual é aquele que contem apenas um ponto aleatório $x$, uniformemente distribuído em um espaço compacto $W \subset \mathbb{R}^{d}$

$$
\mathbf{P}(x \in A)=\frac{\nu_{d}(A)}{\nu_{d}(W)},
$$

Para todos conjuntos borel $A$ contidos em $W$.

Onde, $\nu_{d}($ ) na teoria da medida, é conhecida como a medida de Lebesgue, utilizada para a medida de subconjuntos em um espaço euclidiano $\mathbb{R}^{d}$. A titulo de exemplo, considerando um espaço euclidiano bidimensional $\mathbb{R}^{2}$, $\mathbf{P}(x \in A)$ representa a medida padrão do comprimento da área para o conjunto Borel $A$ contido em $W$.

Assim, considerando $n$ pontos independentes distribuídos uniformemente, eles podem ser superpostos para formar um novo e interessante padrão 
de pontos denominado Processo Pontual Binomial. Usando a definição inicial (A-1), para $n$ pontos independentes $x_{1}, \ldots, x_{n}$, distribuídos uniformemente num espaço compacto $W \subset \mathbb{R}^{d}$, é possível escrever

$$
\begin{array}{r}
\mathbf{P}\left(x_{1} \in A_{1}, \ldots, x_{n} \in A_{n}\right)=\mathbf{P}\left(x_{1} \in A_{1}\right) \ldots \mathbf{P}\left(x_{n} \in A_{n}\right) \\
=\frac{\nu_{d}\left(A_{1}\right) \cdot \ldots \cdot \nu_{d}\left(A_{n}\right)}{\nu_{d}(W)^{n}}
\end{array}
$$

para $n$ conjuntos Borel $A_{1}, \ldots, A_{n}$ contidos em $W$.

Uma definição equivalente, seria dizer que, pontos $x_{1}, \ldots, x_{n}$ formam um processo pontual binomial em $W\left(W \subset \mathbb{R}^{d}\right)$ se o vetor aleatório $\left(x_{1}, \ldots, x_{n}\right)$ é uniformemente distribuído em $W^{n}$. A Figura A.1, ilustra um exemplo do processo pontual Binomial, com $n=100$ pontos.

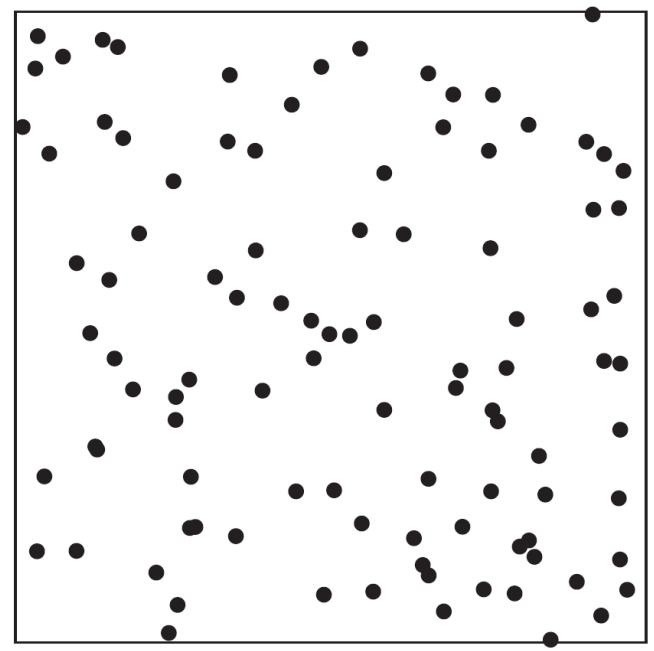

Figura A.1: Exemplo correspondente a um processo pontual Binomial, em $W=[0,1]^{2} \operatorname{com} n=100$ pontos

Assim, o padrão aleatório constituído por esses pontos é indicado por $\Phi_{W^{(n)}}$. Além disso, o processo pontual Binomial pode ser considerado também como uma medida de contagem aleatória (random counting measure), ou seja, para um conjunto Borel $A, \Phi_{W^{(n)}}(A)$ indica o numero de pontos de $\Phi_{W^{(n)}}$ contidos em $A$. Assim,

$$
\Phi_{W^{(n)}}(\emptyset)=0, \quad \Phi_{W^{(n)}}(W)=n
$$

$\mathrm{e}$

$$
\Phi_{W^{(n)}}\left(A_{1} \cup A_{2}\right)=\Phi_{W^{(n)}}\left(A_{1}\right)+\Phi_{W^{(n)}}\left(A_{2}\right),
$$

sempre que $A_{1}$ e $A_{2}$, sejam conjuntos disjuntos de $W$, a propriedade $\sigma$-aditividade também é válida. 
O processo pontual binomial $\Phi_{W^{(n)}}$ é o primeiro exemplo não trivial de um processo pontual. Estas múltiplas visões são importantes para o estudo de todos os processos pontuais aleatórios simples. Assim, um processo pontual é simples se não existem dois pontos que coincidem em um espaço compacto $W$. A simulação do processo pontual Binomial é descrito no Apêndice B.1.

\section{A.2 \\ Processo pontual Poisson Homogêneo}

Um processo pontual $\Phi$, pode ser considerado como uma sequência aleatória, também chamado conjunto aleatório (random set), $\Phi=x_{1}, x_{2}, \ldots$, utilizada como uma medida de contagem aleatória (random counting measure), onde para cada conjunto Borel $B$, o símbolo $\Phi(B)$ representa o número de pontos aleatórios de $\Phi$ que encontram-se contidos dentro do conjunto $B$. Um conjunto aleatório $\Phi$, pode ter interseção com outros conjuntos, e se $B$ é um conjunto Borel, então $B \cap \Phi$ é um conjunto aleatório de pontos $\Phi$ que também pertencem a $B$. Assim, um processo pontual Poisson homogêneo $\Phi$, é caracterizado por duas propriedades fundamentais.

1. Distribuição de Poisson para contagem de pontos (Poisson distribution of point counts), onde o número aleatório de pontos $\Phi$ em um conjunto Borel B tem uma distribuição de Poisson, de média $\lambda \nu_{d}(B)$, para algum valor constante de $\lambda$, isto é

$$
\mathbf{P}(\Phi(B)=m)=\frac{\mu^{m}}{m !} \exp (-\mu) \quad \text { para } m=0,1,2, \ldots
$$

onde, $\mu=\lambda \nu_{d}(B)$.

2. Espalhamento Independente (Independent scattering), os números de pontos de $\Phi$ em $k$ conjuntos Borel disjuntos formam $k$ variáveis aleatórias independentes, para um dado dado $k$ arbitrário. Esta também é conhecida como propriedade completamente aleatória.

Assim, o parâmetro $\lambda$ é um parâmetro característico do processo pontual homogêneo de Poisson que indica o número médio de pontos em um conjunto por unidade de volume, chamado intensidade ou densidade de $\Phi$, sendo $\lambda$ considerado sempre como positivo e finito para todos os conjuntos Borel delimitados por $B$, e satisfaz

$$
\lambda \nu_{d}(B)=\mathbf{E}(\Phi(B))
$$


Entretanto, a propriedade 2 não conduz sempre a 1 . Se a propriedade 1 for enfraquecida, então a propriedade 2 deve ser necessária para caracterizar um processo pontual de Poisson homogêneo. A simulação do processo pontual Poisson Homogêneo é descrito em B.2.

\section{A.3}

\section{Processo pontual Poisson Geral e Não Homogêneo}

O processo pontual de Poisson homogêneo tem uma densidade $\lambda$ constante e uma medida de intensidade que é proporcional a medida de Lebesgue. O número médio de seus pontos por unidade de área não varia no espaço. Entretanto, muitos padrões de pontos que aparecem em algumas aplicações exibem flutuações, e uma aparente tendência de crescimento em uma certa direção, a Figura A.2 ilustra o padrão de pontos que claramente exibem uma dependência direcional.
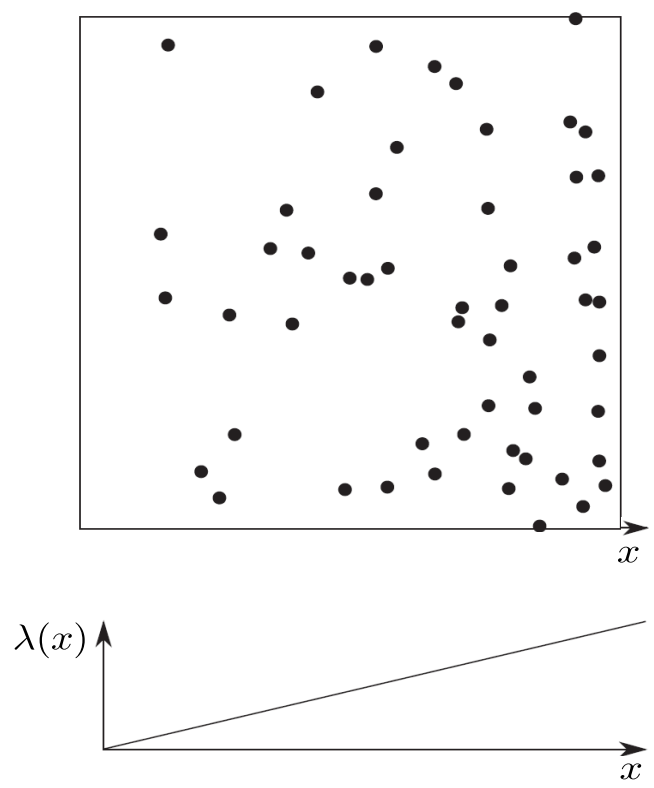

Figura A.2: Exemplo: Processo Pontual de Poisson como função intensidade linearmente crescente $\lambda(x)$

Em tal situação, é possível considerar um modelo de processo pontual como uma função intensidade $\lambda(x)$, e possui uma interpretação infinitesimal intuitiva; $\lambda(x) d x$ é uma probabilidade infinitesimal de encontrar um ponto de $\Phi$ em uma região infinitesimal $d x$, dada por

$$
\Lambda(B)=\int_{B} \lambda(x) d x
$$

onde $\Lambda(B)$, é a medida de intensidade, para um conjunto Borel $B$. 
O processo pontual de Poisson geral $\Phi$, com medida de intensidade $\Lambda$ possui duas propriedades importantes, descritas a seguir

1' Distribuição de Poisson para contagem de pontos (Poisson distribution of point counts), o número de pontos em um conjunto Borel limitado $B$, tem uma distribuição de Poisson com média $\Lambda(B)$

$$
\mathbf{P}(\Phi(B)=n)=\frac{\Lambda(B)^{n}}{n !} \exp (-\Lambda(B)) \quad \text { para } n=0,1,2, \ldots
$$

$2^{\prime}$ Espalhamento Independente (Independent scattering), o número de pontos em $k$ conjuntos Borel disjuntos formam $k$ variáveis aleatórias independentes.

Um processo pontual que possui uma "função intensidade" é chamado processo de Poisson não homogêneo. A simulação do processo pontual Poisson Geral e Não Homogêneo é descrita de forma sucinta em B.2 e B.3.

\section{A.4 \\ Modelos de Processos Pontuais}

Um processo pontual é definido matematicamente como uma variável aleatória, que toma valores em um espaço mensurável $[\mathbb{N}, \mathcal{N}]$, onde $\mathbb{N}$ é uma familia de todas as sequências $\varphi$ de pontos de $\mathbb{R}^{d}$. Formalmente um processo pontual é um mapeamento mensurável em um espaço de probabilidade $[\Omega, \mathcal{A}, \mathbf{P}]$ em $[\mathbb{N}, \mathcal{N}]$. Mais intuitivamente é uma escolha aleatória de uma sequência $\varphi$ em $\mathbb{N}$. O uso da palavra "processo" em termos "processos pontuais" não implica uma evolução dinâmica ao longo do tempo. No entanto, a noção de tempo é muitas vezes ausente em aplicações de processos pontuais em $\mathbb{R}^{2}$ ou $\mathbb{R}^{3}$. O uso da frase "campo pontual aleatório", seria ainda mais exato se: "Processo Pontual = campo pontual aleatório".

\section{A.4.1}

\section{Operações sobre Processos Pontuais}

A construção dos modelos de processos pontuais é uma parte fundamental nas aplicações práticas da geometria estocástica, e são desenvolvidos a partir de estruturas aleatórias simples por meio das três operações fundamentais seguintes:

- Thinning,

- Clustering, e 


\section{- Superposition.}

Em [27],[28],[29] são tratados os teoremas para resultados do repetido uso dessas operações, separadamente e combinadas. Neste Apêndice, somente as próprias operações serão brevemente descritas.

- Thinning: uma operação de thinning usa uma regra definida para apagar pontos de um processo pontual base $\Phi_{b}$, resultando em um processo pontual $\Phi$.

$$
\Phi \subset \Phi_{b}
$$

Uma forma simples de thinning é chamada p-thinning, onde cada ponto $\Phi_{b}$ tem probabilidade $1-p$ de ser apagado, e sua exclusão é independente da localização e da exclusão de qualquer outro ponto de $\Phi_{b}$. Assim, $p$ representa a probabilidade de um ponto seja mantido.

Uma generalização mais natural permite a probabilidade de retenção $p$ depender da localização $x$ dos pontos. Uma função determinística $p(x)$, é dada sobre $\mathbb{R}^{d}$ com $0 \leq p(x) \leq 1$. Se o ponto $x$ pertence a $\Phi_{b}$, ele é excluído com probabilidade $1-p(x)$, e, de novo, sua exclusão é independente da localização, e da exclusão de qualquer outro ponto. Para enfatizar a dependência espacial, a operação generalizada é chamada $p(x)-$ thinning.

Outra generalização permite dependência sobre $\Phi_{b}$, e é chamada classe de thinnings dependente. O qual sugere que um processo pontual é construído por um thinning com a probabilidade de exclusão maior em regiões de alta densidade de pontos. Isto poderia ser obtido, por exemplo, fazendo a probabilidade de exclusão dependente da distância para o ponto mais próximo em um padrão de pontos inicial. A Figura A.3 apresenta o resultado de thinnings independente e dependente aplicado a um mesmo padrão de pontos inicial.

- Clustering: em numa operação de Clustering, cada ponto $x$ de um dado processo pontual $\Phi_{p}$ é substituído por um cluster $N^{x}$ de pontos chamados de pontos filhos. Os $N^{x}$ clusters substituídos são eles processos pontuais que possuem cada um apenas um número finito de pontos. A união de todos esses clusters é um processo pontual cluster $\Phi$.

$$
\Phi=\bigcup_{x \in \Phi_{p}} N^{x}
$$

onde, o índice $p$ indica o ponto 'pai'. 


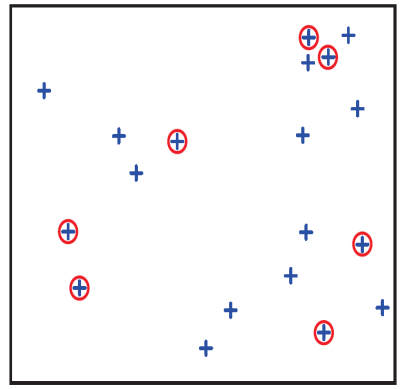

(a)

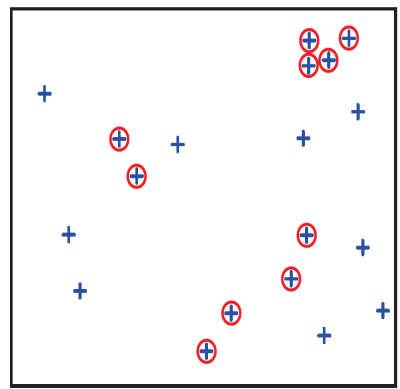

(b)

Figura A.3: Padrões de pontos Thinning, pontos do padrão original são marcados como + , e pontos excluídos $\oplus$. (a) Thinning Independente, realizado por p-thinning. (b) Thinning dependente, exclusão dos pontos com vizinhos mais próximos, com distância $R$.

É assumido que, quase sempre, os pontos de diferentes clusters não coincidem, então $N^{x} \bigcap N^{y}$ é vazio sempre que $x \neq y$. Além disso, é assumido que o processo pontual cluster $\Phi$ é localmente finito. Dependendo do modelo em particular, $x$ pode ou não pertencer a $N^{x}$. A Figura A.4 apresenta um exemplo de um processo pontual cluster.

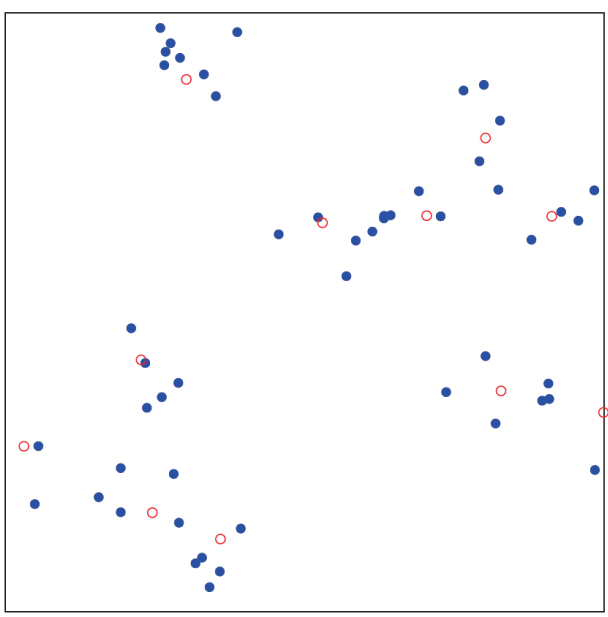

Figura A.4: Exemplo de um processo pontual cluster. Pontos pai são marcados por $\circ$ e pontos filhos por

Processos cluster têm sido utilizados como modelos para muitos fenômenos naturais. Geralmente, os processos cluster servem como modelos de processos pontuais de um grau mais alto de variabilidade que os processos pontuais de Poisson.

- Superposition: seja $\Phi_{1}$ e $\Phi_{2}$ dois processos pontuais com distribuições $P_{1}$ e $P_{2}$, e densidades $\lambda_{1}$ e $\lambda_{2}$, etc. Então, considerando a união

$$
\Phi=\Phi_{1} \cup \Phi_{2},
$$


com probabilidade um. Observe que os conjuntos $\Phi_{1}$ e $\Phi_{2}$, não se sobrepõem, (no caso de independência isto equivale dizer que a correspondente medida de intensidade não possui pontos comuns). A união destes conjuntos coincide com a operação de superposição correspondente à teoria do processo pontual. Fica claro, então, que a medida de intensidade $\Lambda$ e da densidade $\lambda$ de $\Phi$, (no caso de estacionariedade), são dadas por

$$
\begin{array}{r}
\Lambda=\Lambda_{1}+\Lambda_{2}, \\
\lambda=\lambda_{1}+\lambda_{2}
\end{array}
$$

Se $\Phi_{1}$ e $\Phi 2$ são independentes, então a distribuição $P$ de $\Phi$ pode-se escrever por meio do símbolo de convolução *,

$$
P=P_{1} * P_{2}
$$

\section{A.5 \\ Processos Cox}

O processo de Cox é uma generalização do processo de Poisson, feita supondo que a medida de intensidade é aleatória. Tal processo é chamado processo de Poisson duplamente estocástico ou processo Cox. Formalmente, um processo Cox é definido escolhendo uma distribuição $Q$ sobre $[\mathbb{M}, \mathcal{M}]$, o espaço de todas as medidas finitas localmente não negativas sobre $\mathbb{M}^{d}$. Supondo que, $P_{\Lambda}$ é a distribuição do processo de Poisson de medida de intensidade $\Lambda$, e $\Psi$ é uma medida aleatória com distribuição $Q$. Então, o processo Cox $\Phi$ com uma medida aleatória $\Psi$ tem uma distribuição

$$
P_{\Phi}(Y)=\int P_{\Lambda}(Y) Q(d \Lambda) \quad \text { para } Y \in \mathcal{N}
$$

observa-se que $\mathcal{N}$, é o $\sigma$-algebra de $\mathbb{N}$ de todas as sequências de pontos simples e localmente finitos. Para assegurar que $\Phi$, fique simples, e dizer que não tenha pontos múltiplos, assume-se que $Q$ é concentrado sobre subconjuntos de medidas difusas.

Um processo Cox pode ser imaginado como um mecanismo aleatório de dois passos, devido a isso o termo duplamente estocástico. O primeiro passo gera a medida $\Lambda$ sobre $\mathbb{R}^{d}$ de acordo com a distribuição $Q$ da medida aleatória que dirige o processo, e o segundo passo gera um processo Poisson de medida de intensidade $\Lambda$. Portanto eles possuem importantes aplicações como modelos estocásticos. 


\section{A.6 \\ Processos Neyman-Scott}

Processos Neyman-Scott (chamados processos satélite central) são processos cluster de Poisson, frequentemente usado em estatística espacial. Resultam de um clustering independente e homogêneo aplicado a um processo de Poisson homogêneo. Os pontos pais formam um processo de Poisson homogêneo de intensidade $\lambda_{p}$, enquanto os pontos filhos de um cluster representativo $N_{0}$ são aleatórios em número e espalhados com uma densidade de probabilidade espacial $f(y)$, de forma independente e idêntica em torno da origem. Os pontos pais não ocorrem no padrão de pontos observados, somente pontos filhos são incluídos. Utilizando essas premissas, o processo cluster resultante $\Phi$ é estacionário. Se a função densidade de probabilidade $f(y)$, do espalhamento é radialmente simétrico, então $\Phi$ é isotrópico. O processo Neyman-Scott pode ser interpretado como um tipo particular de modelo booleano, onde os clusters tem um papel de grãos.

\section{A.7 \\ Processos Gibss}

Os processos Gibss não são uma classe universal de modelos que pode ser utilizada em todas as situações. Eles são bons modelos para padrões com certo grau de regularidade, até processos cluster moderados, mas podem ser deficientes em casos de forte agrupamento de pontos clustering. A teoria dos processos de Gibbs é desenvolvida a partir da ideia de novos processos pontuais que podem ser gerados não apenas por thinning, clustering e superposição, mas também por transformação de sua distribuição por meio da densidade de probabilidade.

Fundamentalmente a teoria do processo de Gibbs se concentra na ideia de um processo base $\Psi$ de distribuição $Q$. Assim, uma nova distribuição $P$, pode ser definida sobre $[\mathbb{N}, \mathcal{N}]$ por meio de uma densidade de probabilidade $f(\varphi)$ como

$$
P_{\lambda}(Y)=\int_{Y} f(\varphi) Q(d \varphi)
$$

Se $\Phi$, é um processo pontual de distribuição $P$, então $f(\varphi)$ é a probabilidade de $\Phi$ tenha uma realização $\varphi$, que comparada com $\Psi$ seja a mesma realização.

A forma da densidade $f(\varphi)$ é, geralmente, definida pelo campo de aplicação. Ela pode ser escolhida convenientemente para modelar interações 
entre os pontos do processo. A abordagem do processo de Gibbs é direta se o processo a ser modelado contém somente um número de pontos finito e está confinado a uma determinada região limitada. 


\section{B \\ Simulações de Processos Pontuais}

\section{B.1 \\ Simulação do processo pontual Binomial}

Um processo pontual Binomial é facilmente obtido por superposição a partir da simulação de um ponto aleatório $x$ distribuído uniformemente sobre a região requerida $W$, o processo pontual Binomial pode ser obtido por $n$ repetições independentes de pontos aleatórios.

Para simular um ponto aleatório, distribuído uniformemente no intervalo $[0,1]^{2}$. Se $\left\{u_{j}\right\}$ é uma sequência de números aleatórios independentes e uniformemente distribuídos em $[0,1]$, então os pontos

$$
x_{i}=\left(u_{2 i-1}, u_{2 i}\right) \quad \text { para } i=1,2, \ldots
$$

Formam uma sequência de pontos aleatórios independentes, e distribuídos uniformemente no intervalo $[0,1]^{2}$. No caso de um hipercubo, a sequência de pontos aleatórios distribuídos uniformemente em $[0,1]^{d}$ é produzido por

$$
x_{i}=\left(u_{(i-1) d+1}, \ldots, u_{i d}\right) \quad \text { para } i=1,2, \ldots
$$

A simulação para a geração de pontos aleatórios uniformes em uma região delimitada $W$, é realizado através de uma das três principais técnicas

1. Amostragem rejeição (Rejection sampling), um retângulo $R$ contendo $W$ é obtido a partir da geração de uma sequência de pontos aleatórios uniformes e independentes, e simulado no rectângulo $R$ até que um ponto caia em $W$. Este ponto será uniformemente distribuído em $W$. Para obter um processo pontual binomial, este procedimento é repetido até $n$ pontos ter caído em $W$. Para maximizar a eficiência, $R$ deve ser escolhido tão pequeno quanto seja possível. Na Figura (B.1) é apresentada o processo.

2. Aproximação (Approximation), a região $W$ é substituída pela união disjunta de $k$ quadrados abertos, que aproximam $W$. Um ponto aleatório distribuído uniformemente sobre esta união é simulado, primeiro escolhendo um quadrado com probabilidade proporcional a sua área e, então, simulando um ponto aleatório uniformemente distribuído sobre este quadrado. Simulações exatas para regiões complicadas podem ser obtidas a 
partir da combinação desta técnica e a técnica anterior de Rejection sampling.

3. Transformação de Coordenadas (Transformation of coordinates), se a região $W$ exibe alguma simetria, então uma transformação de coordenadas pode ser útil. Assumindo que, se $W$ é um disco unitário $B(o, 1)$, então um ponto aleatório uniforme pode ser descrito em coordenadas polares

$$
x=(r, \theta) \quad \text { para } r \in[0,1] \text { e } \theta \in(0,2 \pi] .
$$

As variáveis aleatórias $r$ e $\theta$ são independentes, com $\theta$ uniforme sobre $(0,2 \pi]$ e $r$ satisfazendo $P(r \leq t)=t^{2}$, se $0 \leq t \leq 1$. Assim se $u_{1}$ e $u_{2}$ são números aleatórios independentes e uniformes sobre $[0,1]$, então

$$
r=\sqrt{u_{1}} \quad \text { e } \quad \theta=2 \pi u_{2}
$$

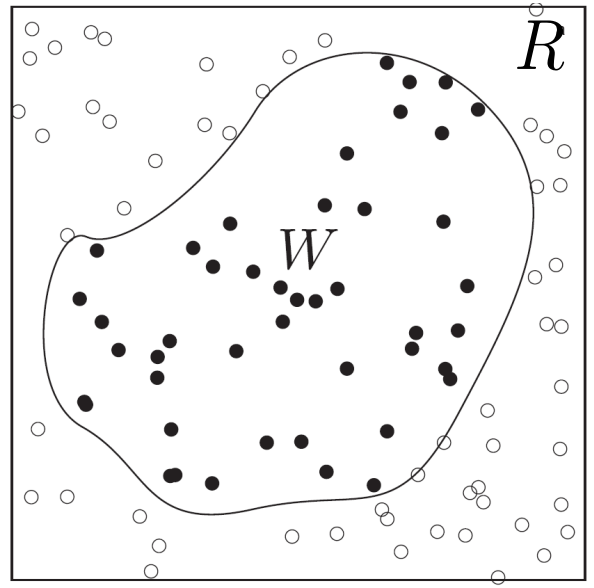

Figura B.1: Simulação de 45 pontos aleatórios uniformemente distribuídos em um região $W$.

\section{B.2}

\section{Simulação do processo pontual Poisson homogêneo}

Considerando uma região compacta $W$, a simulação é feita em dois estágios. No primeiro estagio, o número de pontos em $W$ é determinado por meio da simulação de uma variável aleatória de Poisson. No segundo estagio, as posições dos pontos em $W$ são determinados por meio da simulação de um processo pontual Binomial que utiliza aquele número de pontos gerados no primeiro estagio. O procedimento para o desenvolvimento do segundo estagio 
foi obtido a partir do item (1) em B.1. Existem várias possibilidades para a geração do primeiro estágio, ou seja a simulação de uma variável aleatória de Poisson, o método mais utilizado é aquele que depende da média $\mu=\lambda \nu_{d}(W)$. Este método é geralmente utilizado para pequenos valores de $\mu$, e considera o processo de Poisson como linear, explorando o fato que suas distâncias entre pontos sejam variáveis aleatórias exponenciais independentes [25].

\section{B.3}

\section{Simulação do Processo pontual Poisson Geral Não Homogêneo}

Para simular uma amostra de um processo pontual de Poisson não homogêneo com função intensidade $\lambda(x)$, um procedimento de filtragem (thinning) pode ser usado $[25,26]$. O procedimento assume que a função intensidade é limitada acima de um número $\lambda^{*}$, sendo executada em dois estágios. Primeiro, um processo pontual de Poisson homogêneo de intensidade $\lambda^{*}$ é simulado como mostrado acima. Segundo, o padrão de pontos resultante é filtrado, excluindo cada ponto $x$ independentemente dos outros com probabilidade $1-\lambda(x) / \lambda^{*}$. Se os pontos do padrão do processo pontual Poisson homogêneo são $\left\{x_{1}, x_{2}, \ldots\right\}$ então esta filtragem pode ser realizada com a ajuda de uma sequência $u_{1}, u_{2}, \ldots$ de números aleatórios independentes e uniformemente distribuídos em $[0,1]$. O ponto $x_{k}$ é excluído se $u_{k}>\lambda\left(x_{k}\right) / \lambda^{*}$.

\section{B.4 \\ Simulação do Processo Pontual de Poisson (PPP) homogéneo sobre as regiões geográficas definidas na Subseção 4.1.1}

A técnica utilizada neste trabalho para gerar os padrões de pontos é a denominada Amostragem rejeição (Rejection sampling), a modo de exemplificar a aplicabilidade da simulação para gerar pontos (terminais) aleatórios distribuídos uniformemente (padrões de pontos) usando o processo pontual Poisson homogêneo, são consideradas seis regiões geográficas $\left\{W_{m}, m=1, \ldots, 6\right\}$ e definidas na Subseção 4.1.1. A área correspondente a cada rectângulo, contendo cada região definida como $\left\{R_{m}, m=1, \ldots, 6\right\}$, com uma densidade $\lambda$, $\left\{\lambda_{m}, m=1, \ldots, 6\right\}$, aqui são utilizadas para gerar o processo pontual de Poisson (PPP). O número de pontos contidos em cada região $W_{m}$ é determinado por simulação e descrito neste Apêndice. A Tabela B.1, apresenta um resumo dos parâmetros correspondentes de cada região $W$, a área do rectângulo $R \mathrm{e}$ densidade $\lambda$ de pontos por cada região, utilizados para gerar o processo pontual Poisson (PPP) homogêneo. 
Tabela B.1: Parâmetros $R, W$ e $\lambda$. Assumindo uma rede com $\mathcal{N}_{T}=211$ terminais

\begin{tabular}{|c|c|c|c|}
\hline \hline$m$ & Área da região $W_{m}\left(\mathrm{~km}^{2}\right)$ & Área do rectângulo $R_{m}\left(\mathrm{~km}^{2}\right)$ & $\lambda_{m}$ \\
\hline 1 & 747.075 & 5.703 .136 & $1,127 \times 10^{-4}$ \\
\hline 2 & 1.172 .461 & 3.565 .346 & $5,809 \times 10^{-6}$ \\
\hline 3 & 486.807 & 1.944 .892 & $1,338 \times 10^{-4}$ \\
\hline 4 & 725.217 & 3.968 .744 & $1,248 \times 10^{-5}$ \\
\hline 5 & 335.342 & 1.429 .949 & $2,917 \times 10^{-5}$ \\
\hline 6 & 5.101 .773 & 11.385 .188 & $7,054 \times 10^{-6}$ \\
\hline \hline
\end{tabular}

As figuras B.2 e B.3 ilustram a modo de exemplo a simulação de um processo pontual Poisson (PPP), para a Região 1 e Região 6 respectivamente.

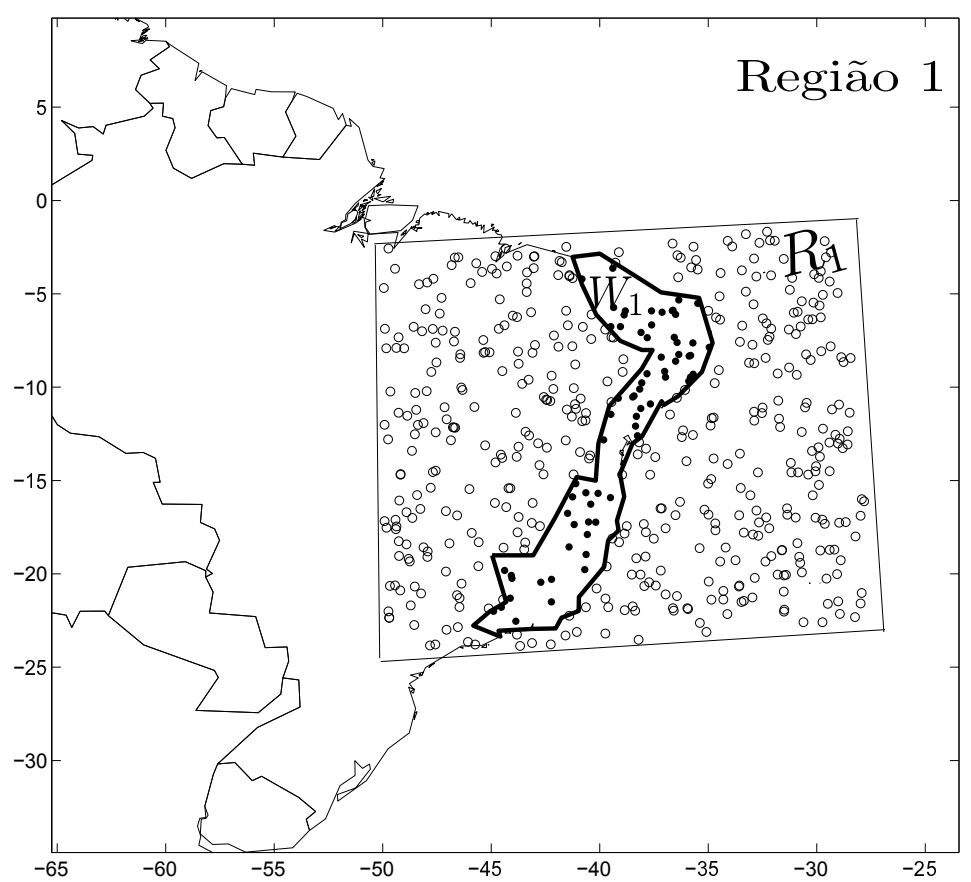

Figura B.2: Simulação de um Processo pontual de Poisson(PPP), uniformemente distribuído correspondente à Região 1. 


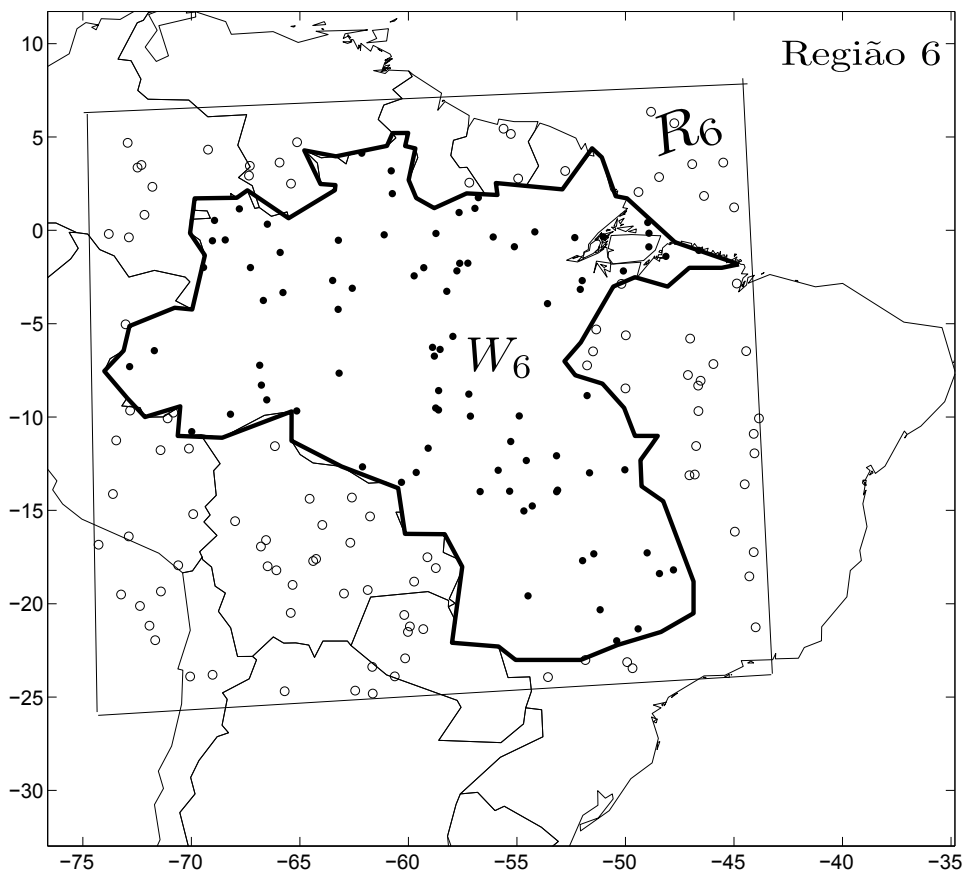

Figura B.3: Simulação de um Processo pontual de Poisson(PPP), uniformemente distribuído correspondente à Região 6 . 


\section{C \\ Diagrama de radiação das antenas correspondentes aos ter- minais interferentes $T^{\prime}$ da rede VSAT: Recomendação ITU-R F.1245-1}

A Recomendação ITU-R F.1245-1, fornece padrões de radiação de referência média para antenas do sistema fixo sem fio ponto-a-ponto, na faixa de frequência de $1 \mathrm{GHz}$ a $70 \mathrm{GHz}$. O modelo matemático do diagrama de radiação é usado para frequências na faixa de $1 \mathrm{GHz}$ a $40 \mathrm{GHz}$ e provisoriamente na faixa de $40 \mathrm{GHz}$ a $70 \mathrm{GHz}$. Para antenas transmissoras dos terminais VSAT interferentes de 0,96, 1,20 e 1,80 metros considerou-se o Caso 2.2 da Recomendação ITU-R F.1245-1, onde a razão entre o diâmetro da antena $d$ e o cumprimento de onda é menor ou igual a $100(d / \lambda \leq 100)$, com diagrama de radiação descrito em (C-1).

$$
\begin{array}{lll}
G(\varphi)=G_{\max }-2.5 \times 10^{-3}\left(\frac{d}{\lambda} \varphi\right) & ; 0 \leq \varphi<\varphi_{m} \\
G(\varphi)=39-5 \log (d / \lambda)-25 \log \varphi & ; \quad \varphi_{m} \leq \varphi<48^{\circ} \\
G(\varphi)=-3-5 \log (d / \lambda) & ; \quad 48^{\circ} \leq \varphi<180^{\circ}
\end{array}
$$

Alguns, dos parâmetros técnicos para o Caso 2.2 da Recomendação ITUR F.1245-1, são apresentados na Tabela C.1, e os respectivos diagramas de radiação são ilustrados na Figura C.1 .

Tabela C.1: Parâmetros correspondentes às antenas dos terminais interferentes $T^{\prime}$ VSAT.

\begin{tabular}{|l|r|r|r|}
\hline \hline & Antena 1 & Antena 2 & Antena 3 \\
\hline Diâmetro $[\mathrm{m}]$ & 0,96 & 1,2 & 1,8 \\
\hline Frequência $[\mathrm{GHz}]$ & 14 & 14 & 14 \\
\hline Eficiência $(\eta)$ & 0,55 & 0,55 & 0,55 \\
\hline Ganho máximo [dBi] & 40,37 & 42,31 & 45,83 \\
\hline$d / \lambda$ & 44,80 & 56,00 & 84,00 \\
\hline Ganho primeiro lóbulo [dBi] & 26,77 & 28,22 & 30,86 \\
\hline$\varphi_{m}$ [graus] & 1,65 & 1,34 & 0,92 \\
\hline$\varphi_{r}$ [graus] & 1,22 & 1,07 & 0,84 \\
\hline \hline
\end{tabular}


Apêndice C. Diagrama de radiação das antenas correspondentes aos terminais interferentes $T^{\prime}$ da rede VSAT: Recomendação ITU-R F.1245-1

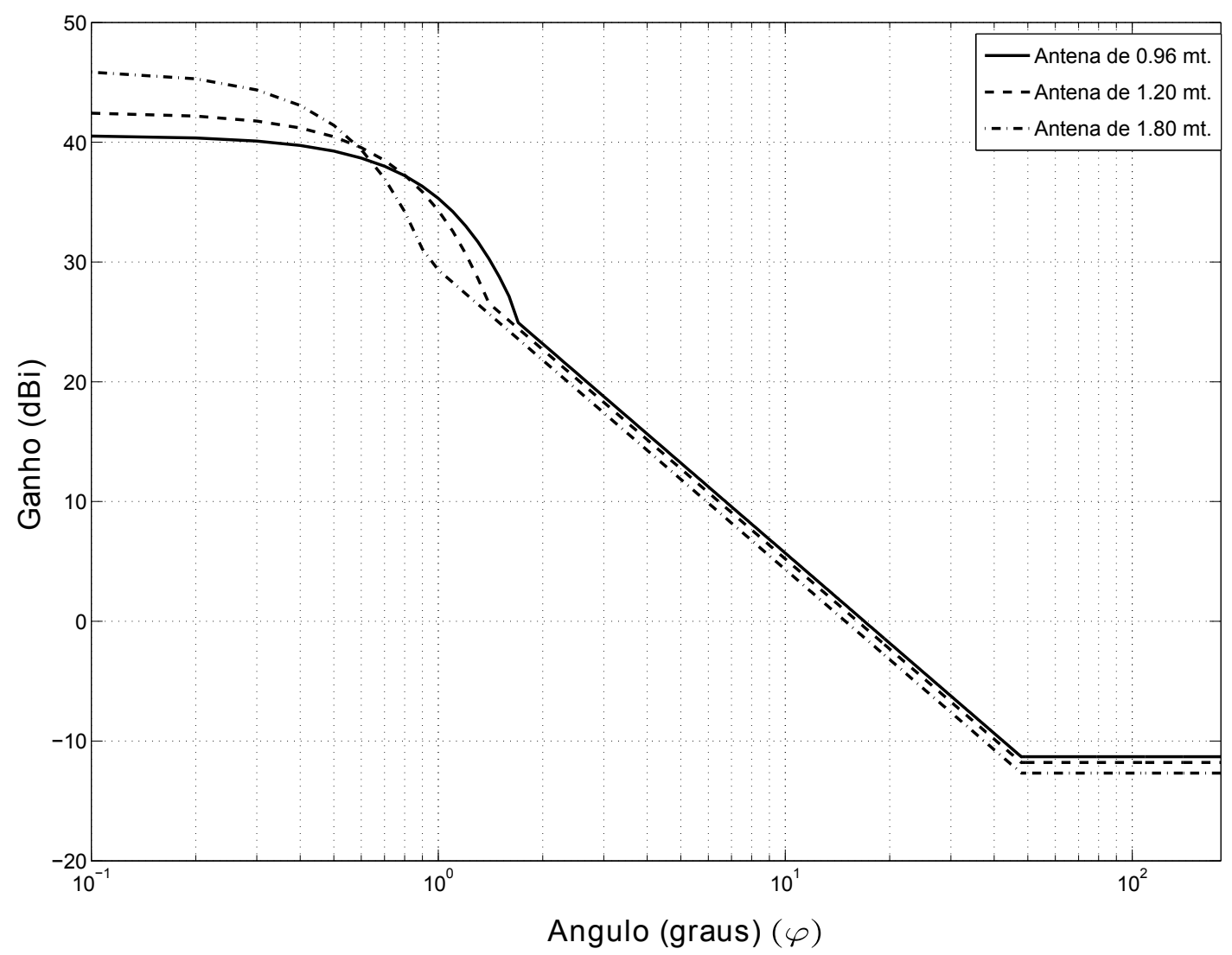

Figura C.1: Diagramas de radiação das antenas dos terminais interferentes $T^{\prime}$, da rede VSAT MF-TDMA. 
D

Diagrama de radiação da antena receptora do satélite vitima $S$ : Recomendação ITU-R S.672-4

Considerou-se o padrão de radiação de referencia da antena do satélite do Anexo 1, correspondente ao feixe circular contida na Recomendação ITUR S.672-4. Assim, os diagramas possíveis começam no contorno de $-3 \mathrm{~dB}$ do lóbulo principal e são divididos em quatro regiões, como é ilustrada na Figura D.1.

O diagrama de radiação da antena, contém as seguintes funções

$$
\begin{array}{ll}
G(\Psi)=G_{m}-3\left(\Psi / \Psi_{0}\right)^{2} & ; \Psi_{0} \leq \Psi<a \Psi_{0} \\
G(\Psi)=G_{m}+L_{s} & ; a \Psi_{0} \leq \Psi<b \Psi_{0} \\
G(\Psi)=G_{m}+L_{s}+20-25 \log \left(\Psi / \Psi_{0}\right) & ; b \Psi_{0} \leq \Psi<\Psi_{1} \\
G(\Psi)=0 & ; \Psi_{1} \leq \Psi
\end{array}
$$

Tabela D.1: Parâmetros da antena receptora do satélite $S$.

\begin{tabular}{|l|r|}
\hline \hline & Antena \\
\hline Diâmetro $(\mathrm{m})$ & 1,4 \\
\hline Frequência $(\mathrm{GHz})$ & 14 \\
\hline Eficiência $(\eta)$ & 0,55 \\
\hline Ganho máximo $\left(G_{m}\right)(\mathrm{dBi})$ & 30,99 \\
\hline$\theta_{3 d B}$ (graus) & 6 \\
\hline$\Psi_{0}$ (graus) & 3 \\
\hline$L_{s}(\mathrm{~dB})$ & -30 \\
\hline$a$ & 3,16 \\
\hline$b$ & 6,32 \\
\hline \hline
\end{tabular}


Apêndice D. Diagrama de radiação da antena receptora do satélite vitima $S$ : Recomendação ITU-R S.672-4

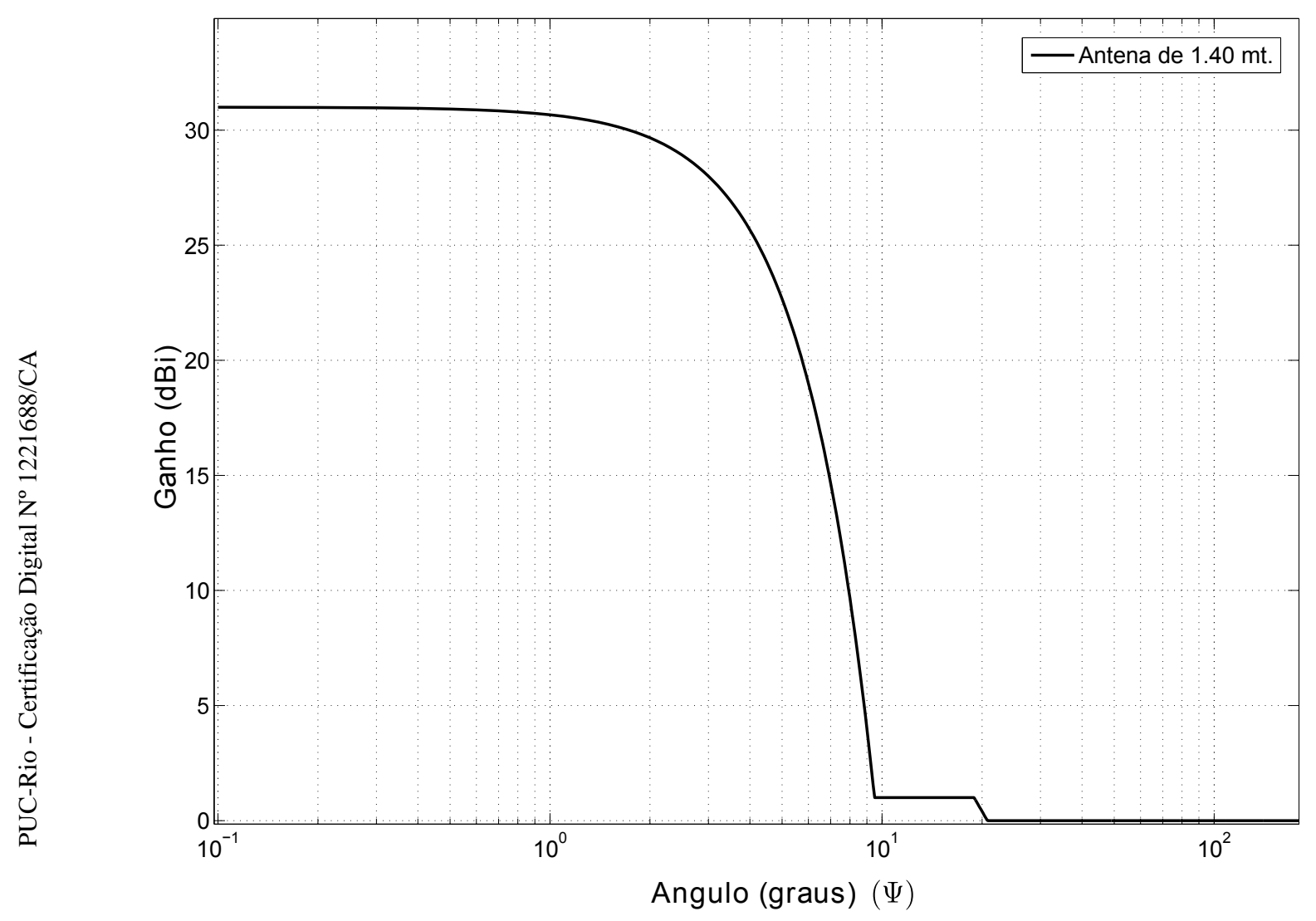

Figura D.1: Diagrama de radiação da antena receptora do satélite vítima $S$. 


\section{E \\ Níveis máximos admissíveis da densidade e.i.r.p., das antenas transmissoras VSAT, Recomendação ITU-R S.728-1}

Na Recomendação ITU-R S.728-1 são estabelecidos níveis máximos admissíveis da densidade e.i.r.p. off-axis, que deverão ser utilizados por cada estação terrena transmissora VSAT, com o objetivo de limitar o uso da potencia interferente em redes de satélites geoestacionários (GSO). Estes níveis máximos admissíveis da densidade e.i.r.p., são definidos para antenas transmissoras que utilizam a bandas de frequência de $14 \mathrm{GHz}$.

A Recomendação ITU-R S.728-1 estabelece que, as estações que transmitam utilizando redes de satélites geoestacionários (GSO) e sistemas fixos terrestres (SFS), na banda de frequências de $14 \mathrm{GHz}$, são desenhadas de modo que, para quaisquer ângulo $\varphi$, fora do eixo do lóbulo principal da antena correspondente à estação terrena, a densidade e.i.r.p. em qualquer direção dentro dos $3^{\circ}$ não deverá exceder os valores seguintes,

$$
\begin{array}{ll}
\text { e.i.r.p.e máximo para } 40 \mathrm{KHz} & \text { Angulo (Off-axis) } \\
(33-25 \log \varphi) \mathrm{dB}(\mathrm{W} / 40 \mathrm{KHz}) & ; 2^{\circ} \leq \varphi \leq 7^{\circ} \\
12 \mathrm{~dB}(\mathrm{~W} / 40 \mathrm{KHz}) & ; 7^{\circ}<\varphi \leq 9.2^{\circ} \\
(36-25 \log \varphi) \mathrm{dB}(\mathrm{W} / 40 \mathrm{KHz}) & ; \quad 9.2^{\circ}<\varphi \leq 48^{\circ} \\
-6 \mathrm{~dB}(\mathrm{~W} / 40 \mathrm{KHz}) & ; \varphi>48^{\circ}
\end{array}
$$

Os possíveis valores da densidade e.i.r.p., calculados para os três diâmetros de antenas $\left(d_{j}, j=1,2,3\right)$ utilizados foram descritos na Tabela 4.3. Para que esses níveis de densidade de e.i.r.p. transmitidos satisfaçam ao limite máximo permitido na Recomendação ITU-R S.728-1 [12], os valores das curvas correspondentes a cada diagrama de radiação não deverão ultrapassar (tangenciar) à curva definida em (E-1). Observe-se na Figura ??, que em cada caso, os padrões de radiação das antenas sempre tangenciam à curva da Recomendação ITU-R S.728-1. Assim, os níveis de densidade de potência de transmissão de uma antena de diâmetro 0,96, 1,20 ou 1,80 metros foi determinado como sendo o maior valor valor de potência $P_{j}$ que satisfaz à condição em cada caso.

$$
P_{j} g_{j}(\varphi) \leq L_{728}(\varphi) \quad \forall \varphi \in\left[2^{\circ}, 180^{\circ}\right]
$$

onde, $L_{728}(\varphi)$ representa valores dos níveis máximos admissíveis da densidade e.i.r.p. estabelecida na Recomendação ITU-R S.728-1. Considerou-se também, 
Apêndice E. Níveis máximos admissíveis da densidade e.i.r.p., das antenas transmissoras VSAT, Recomendação ITU-R S.728-1

uma normalização dos valores do padrão da densidade de e.i.r.p. definido em (E-1), como dB(W/40KHz) para $\mathrm{dB}(\mathrm{W} / 1 \mathrm{~Hz})$.

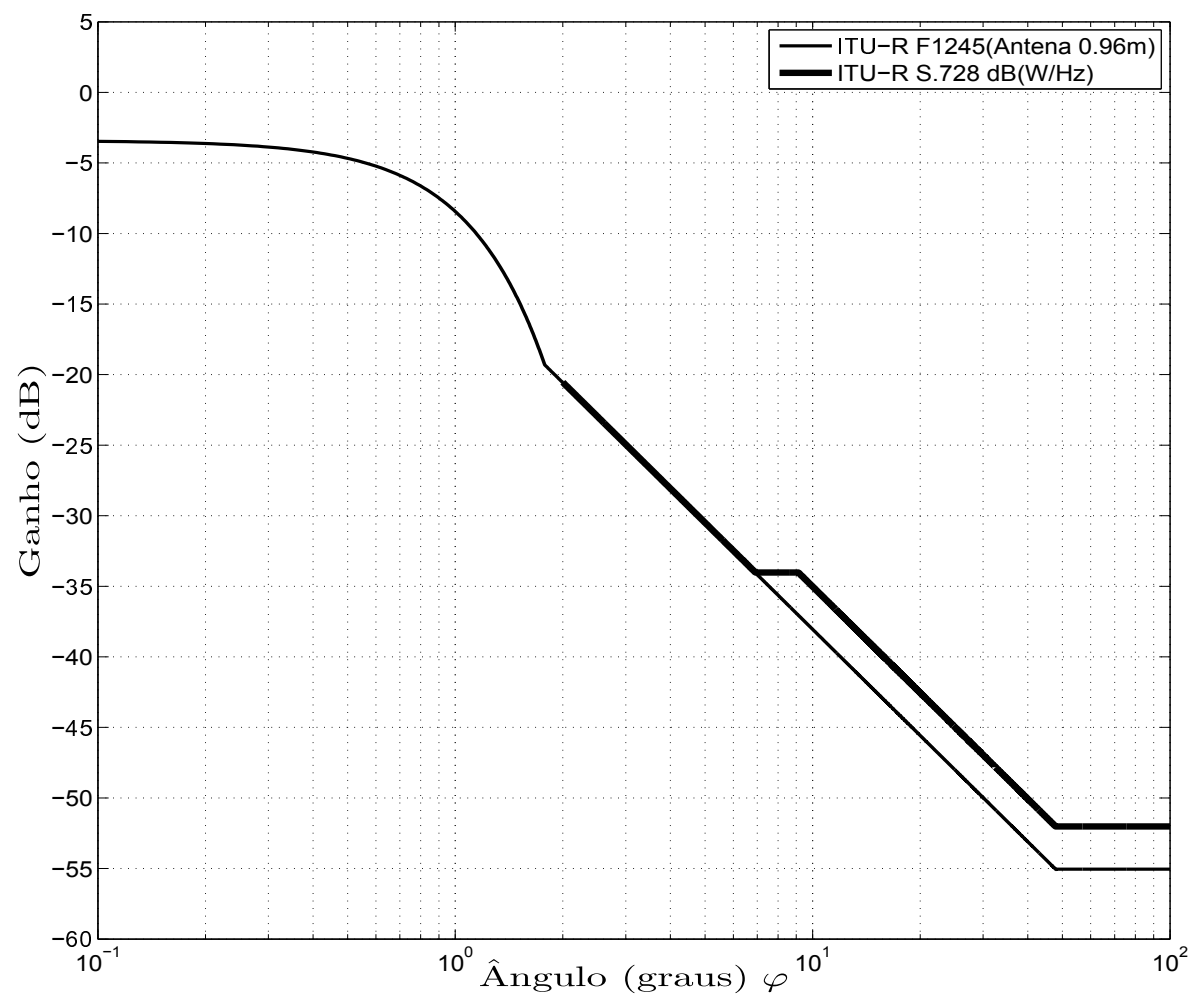

Figura E.1: Diagrama de radiação antena 0,96 m. e nível máximo admissível da densidade e.i.r.p.e (Recomendação ITU-R S.728-1) em dB(W/Hz) 


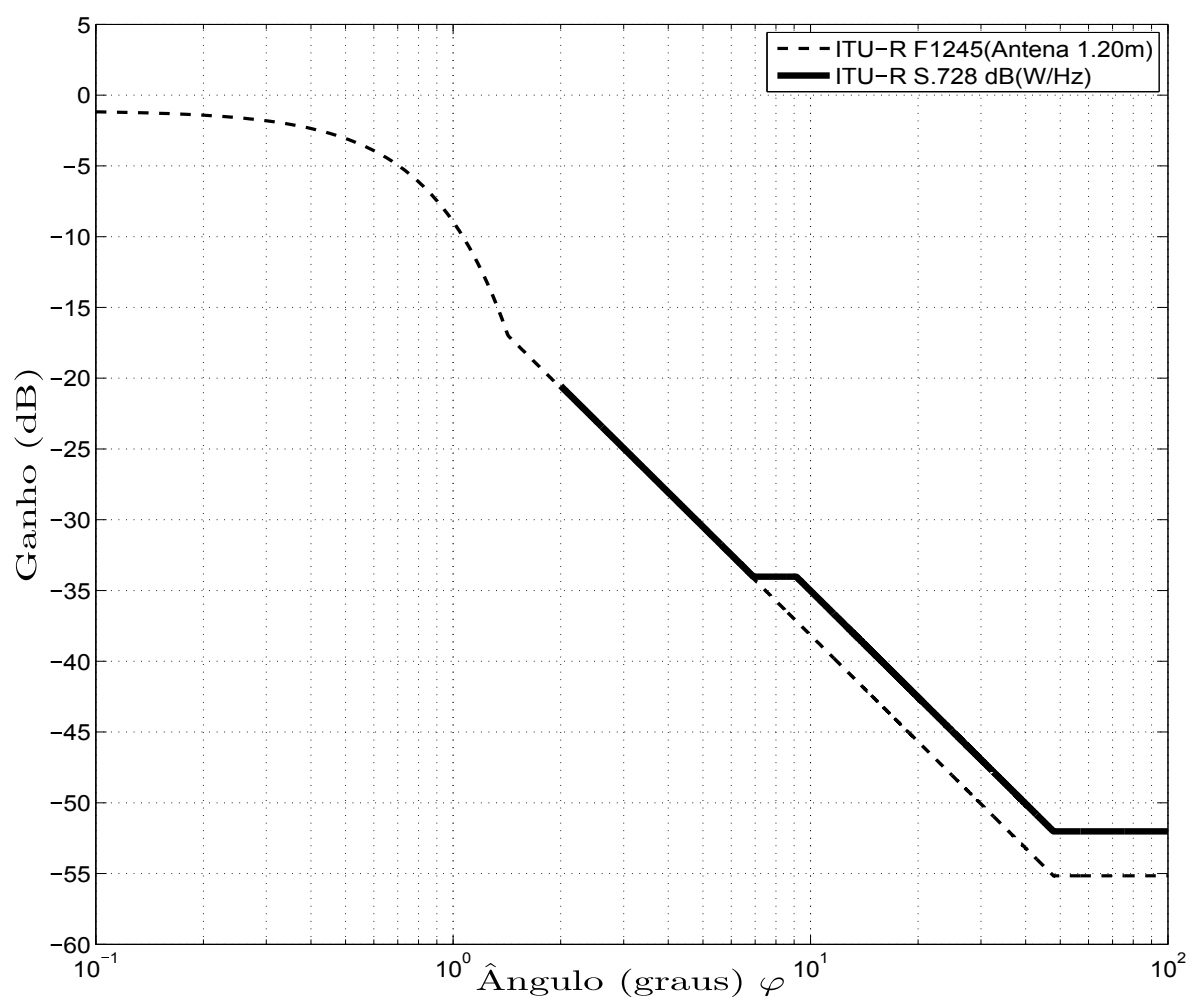

Figura E.2: Diagrama de radiação antena 1,20 m. e nível máximo admissível da densidade e.i.r.p.e (Recomendação ITU-R S.728-1) em dB(W/Hz)

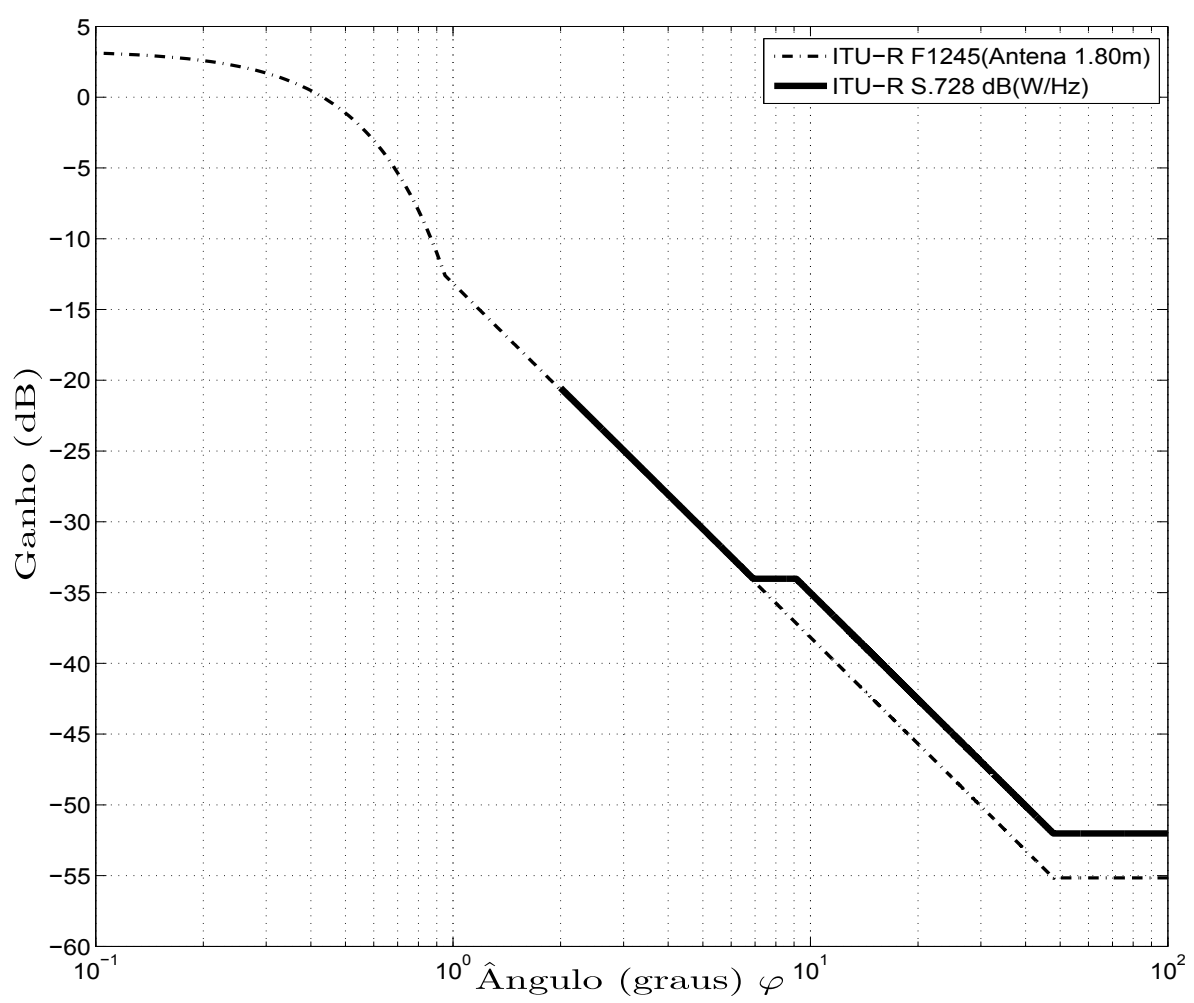

Figura E.3: Diagrama de radiação antena 1,80 m. e nível máximo admissível da densidade e.i.r.p.e (Recomendação ITU-R S.728-1) em dB(W/Hz) 


\section{$\mathbf{F}$}

Comportamento estatístico da Função distribuição de probabilidade complementar correspondente por região $\mathcal{R}_{m},(m=$ $1 \ldots 6)$, para a rede 1 e 2 com $R_{r e f}\left(54,90^{\circ} \mathbf{W}, 9,70^{\circ} \mathbf{S}\right)$

\section{F.1}

Comparação do comportamento estatístico da variável aleatória $i_{n}$ obtida a partir do modelo proposto e estimativas a partir de dados reais da operadora de satélites Embratel Star One, por região $\mathcal{R}_{m},(m=1 \ldots 6)$ correspondente à Rede 1 de 211 terminais VSAT.

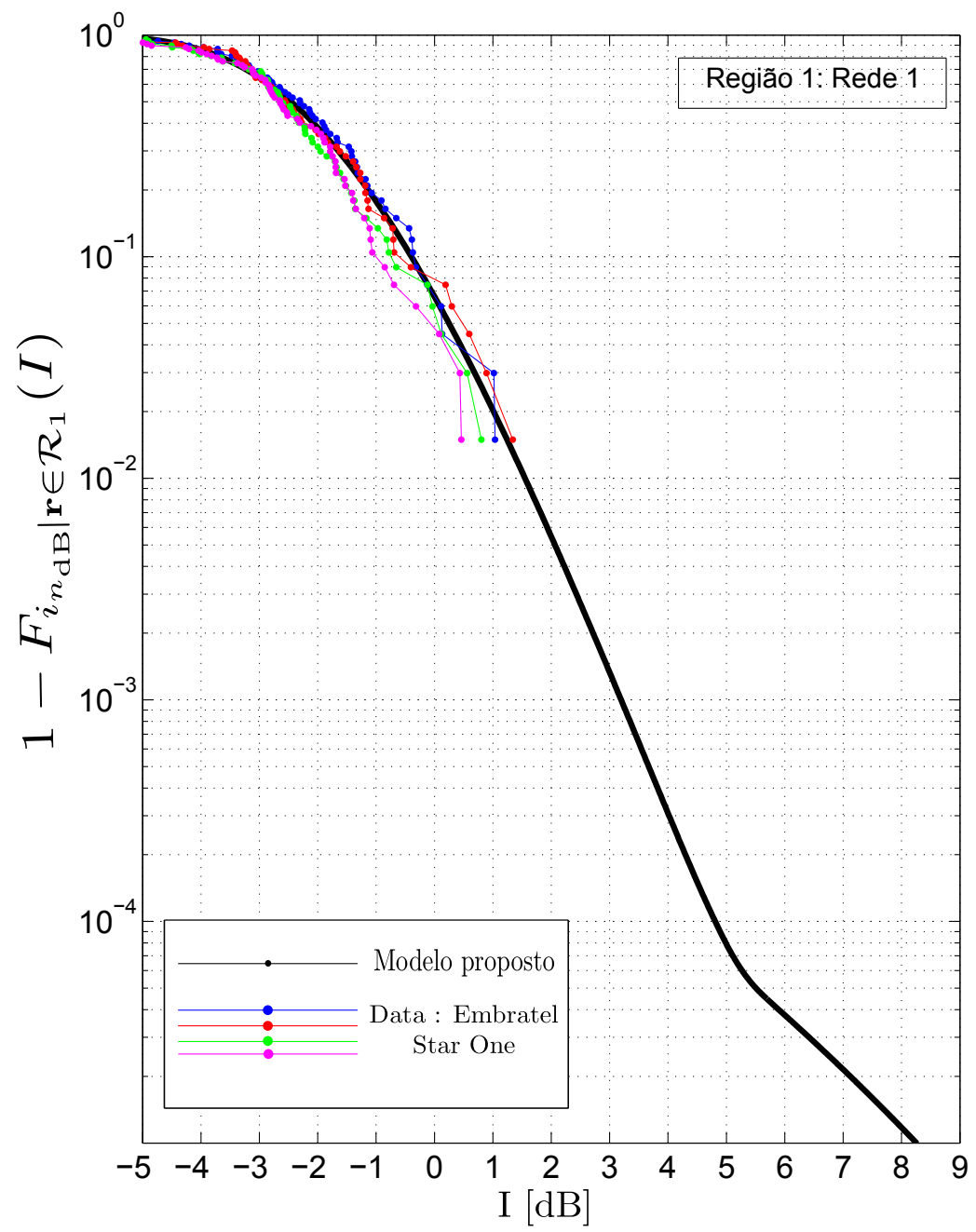

Figura F.1: Número de terminais $\mathcal{N}_{m}: 84$. 
Apêndice F. Comportamento estatístico da Função distribuição de probabilidade complementar correspondente por região $\mathcal{R}_{m},(m=1 \ldots 6)$, para a rede 1 e 2 com $R_{\text {ref }}\left(54,90^{\circ} \mathrm{W}, 9,70^{\circ} \mathrm{S}\right)$



Figura F.2: Número de terminais $\mathcal{N}_{m}: 7$. 
Apêndice F. Comportamento estatístico da Função distribuição de probabilidade complementar correspondente por região $\mathcal{R}_{m},(m=1 \ldots 6)$, para a rede 1 e 2 com $R_{\text {ref }}\left(54,90^{\circ} \mathrm{W}, 9,70^{\circ} \mathrm{S}\right)$

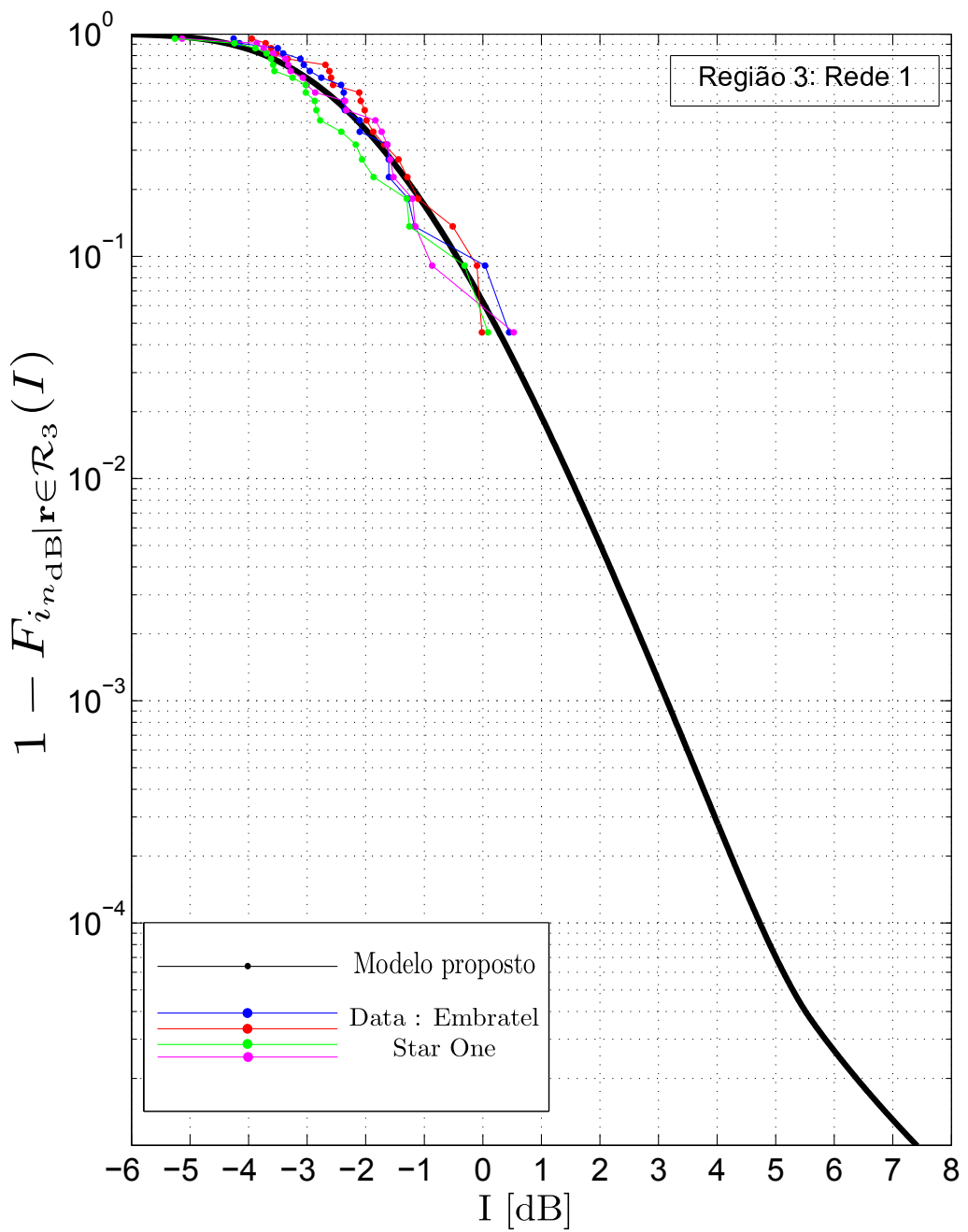

Figura F.3: Número de terminais $\mathcal{N}_{m}: 65$. 
Apêndice F. Comportamento estatístico da Função distribuição de probabilidade complementar correspondente por região $\mathcal{R}_{m},(m=1 \ldots 6)$, para a rede 1 e 2 com $R_{\text {ref }}\left(54,90^{\circ} \mathrm{W}, 9,70^{\circ} \mathrm{S}\right)$

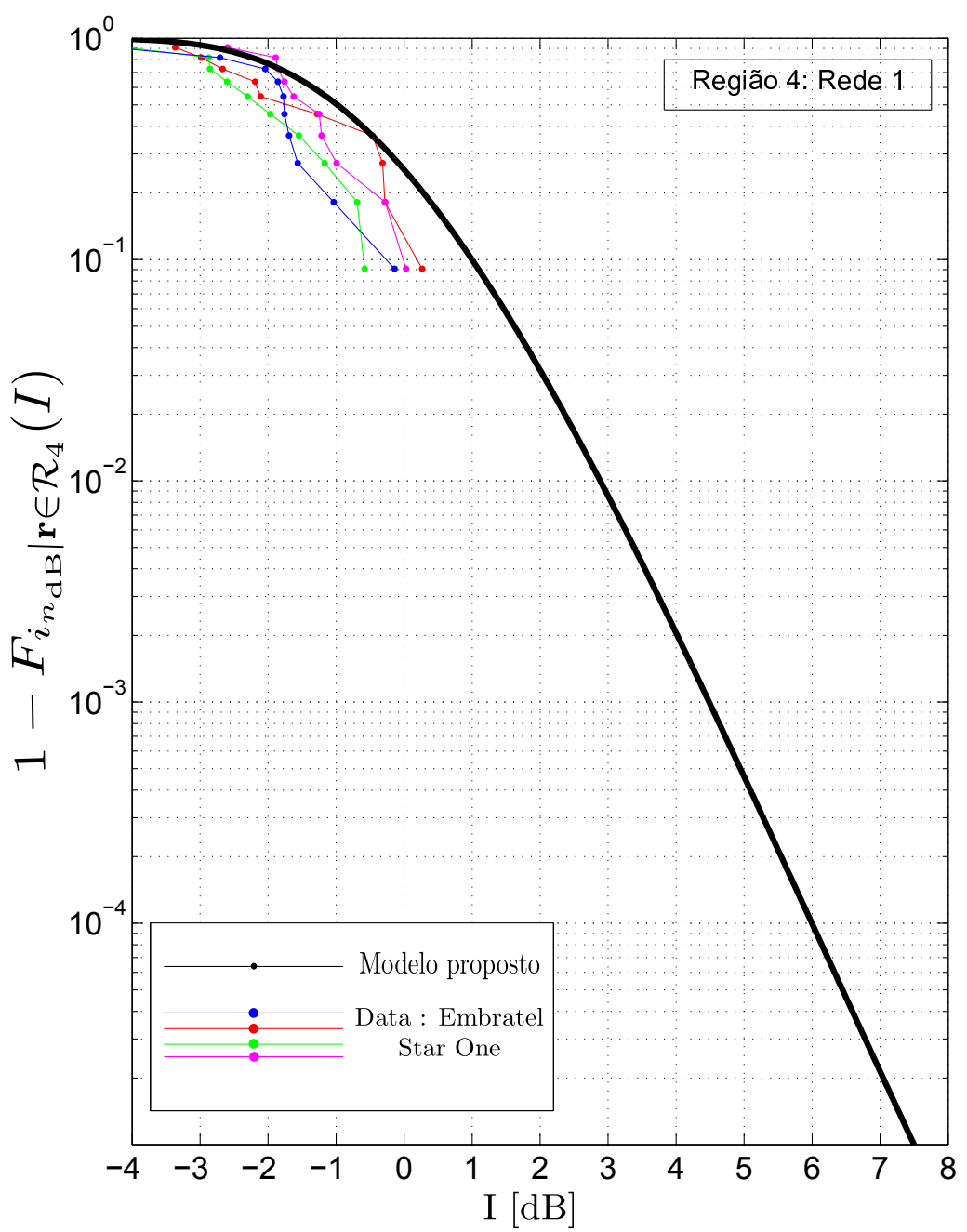

Figura F.4: Número de terminais $\mathcal{N}_{m}: 9$. 
Apêndice F. Comportamento estatístico da Função distribuição de probabilidade complementar correspondente por região $\mathcal{R}_{m},(m=1 \ldots 6)$, para a rede 1 e 2 com $R_{\text {ref }}\left(54,90^{\circ} \mathrm{W}, 9,70^{\circ} \mathrm{S}\right)$

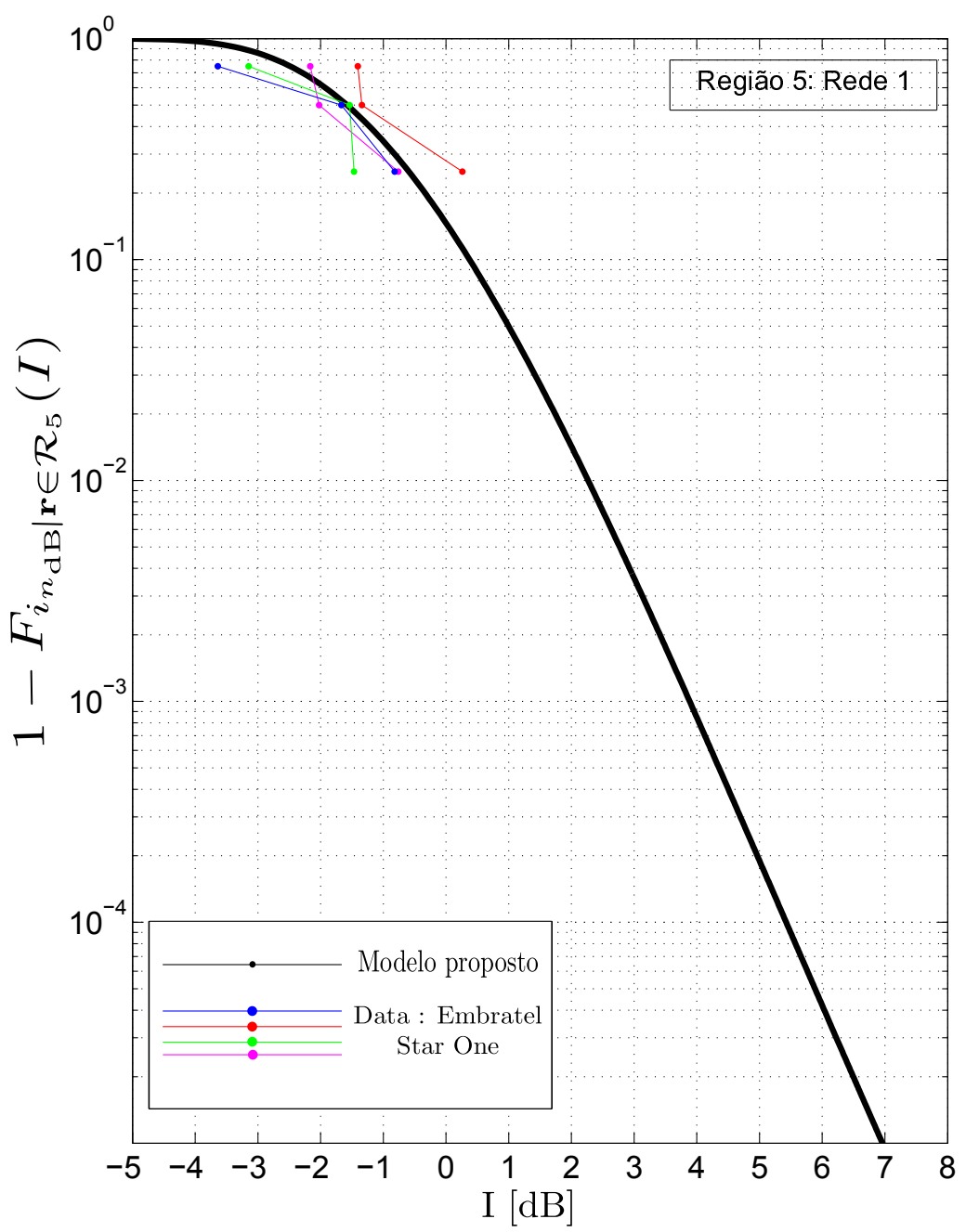

Figura F.5: Número de terminais $\mathcal{N}_{m}: 10$. 
Apêndice F. Comportamento estatístico da Função distribuição de probabilidade complementar correspondente por região $\mathcal{R}_{m},(m=1 \ldots 6)$, para a rede 1 e 2 com $R_{\text {ref }}\left(54,90^{\circ} \mathrm{W}, 9,70^{\circ} \mathrm{S}\right)$

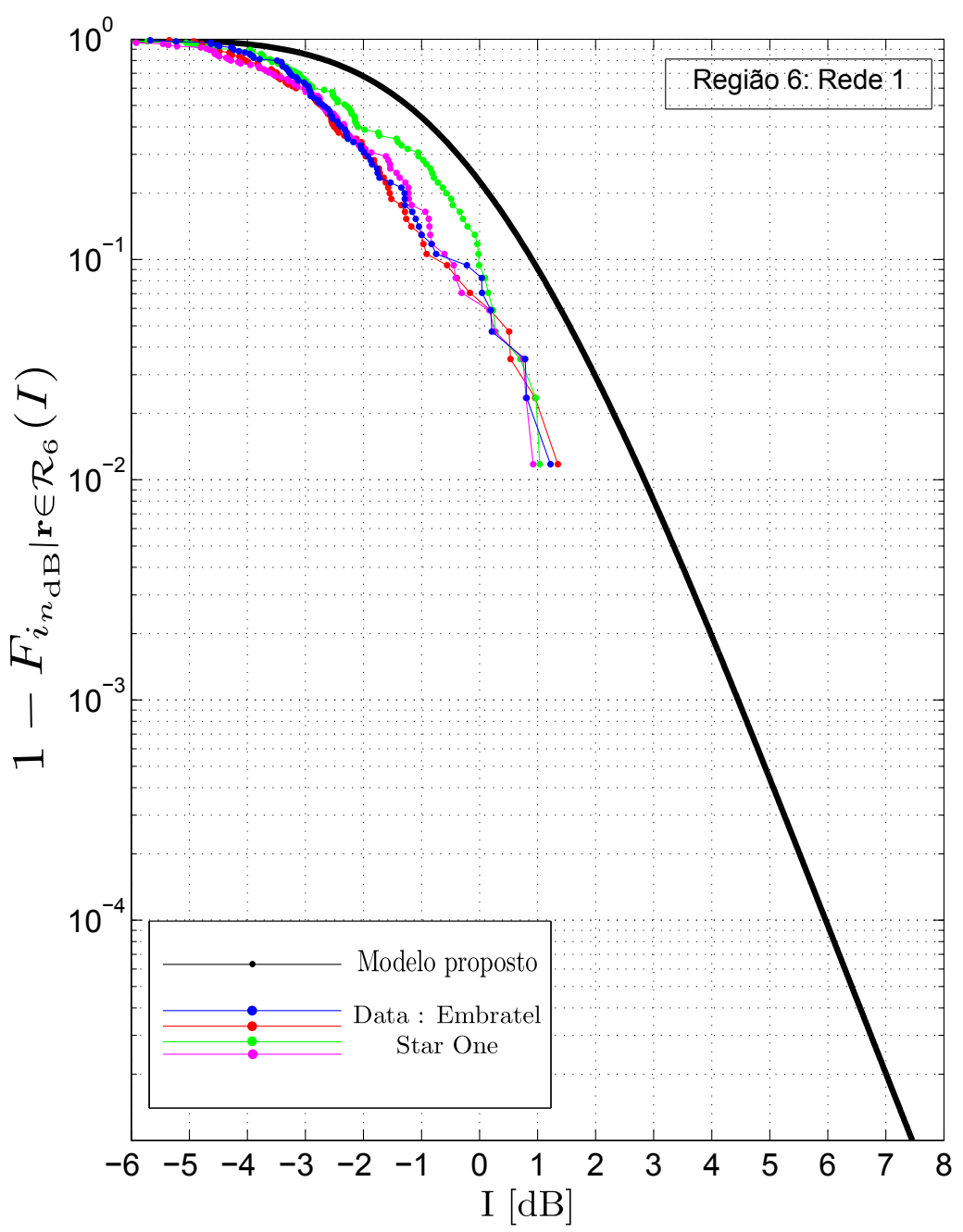

Figura F.6: Número de terminais $\mathcal{N}_{m}: 36$. 
Apêndice F. Comportamento estatístico da Função distribuição de probabilidade complementar correspondente por região $\mathcal{R}_{m},(m=1 \ldots 6)$, para a rede 1 e 2 com $R_{\text {ref }}\left(54,90^{\circ} \mathrm{W}, 9,70^{\circ} \mathrm{S}\right)$

F.2

Comparação do comportamento estatístico da variável aleatória $i_{n}$ obtida a partir do modelo proposto e estimativas a partir de dados reais da operadora de satélites Embratel Star One, por região $\mathcal{R}_{m},(m=1 \ldots 6)$ correspondente à Rede 2 de 78 terminais VSAT.

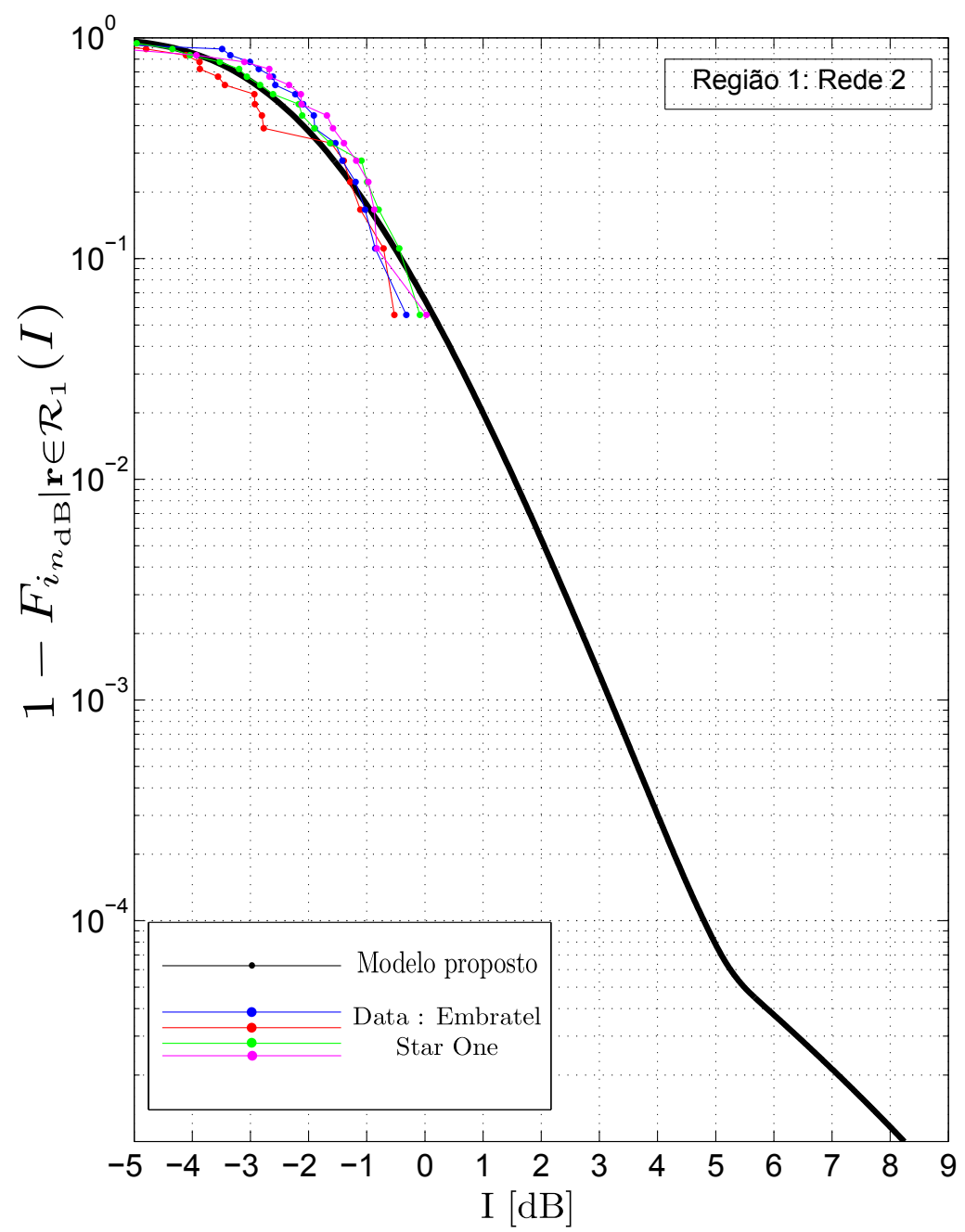

Figura F.7: Número de terminais $\mathcal{N}_{m}: 31$. 
Apêndice F. Comportamento estatístico da Função distribuição de probabilidade complementar correspondente por região $\mathcal{R}_{m},(m=1 \ldots 6)$, para a rede 1 e 2 com $R_{\text {ref }}\left(54,90^{\circ} \mathrm{W}, 9,70^{\circ} \mathrm{S}\right)$

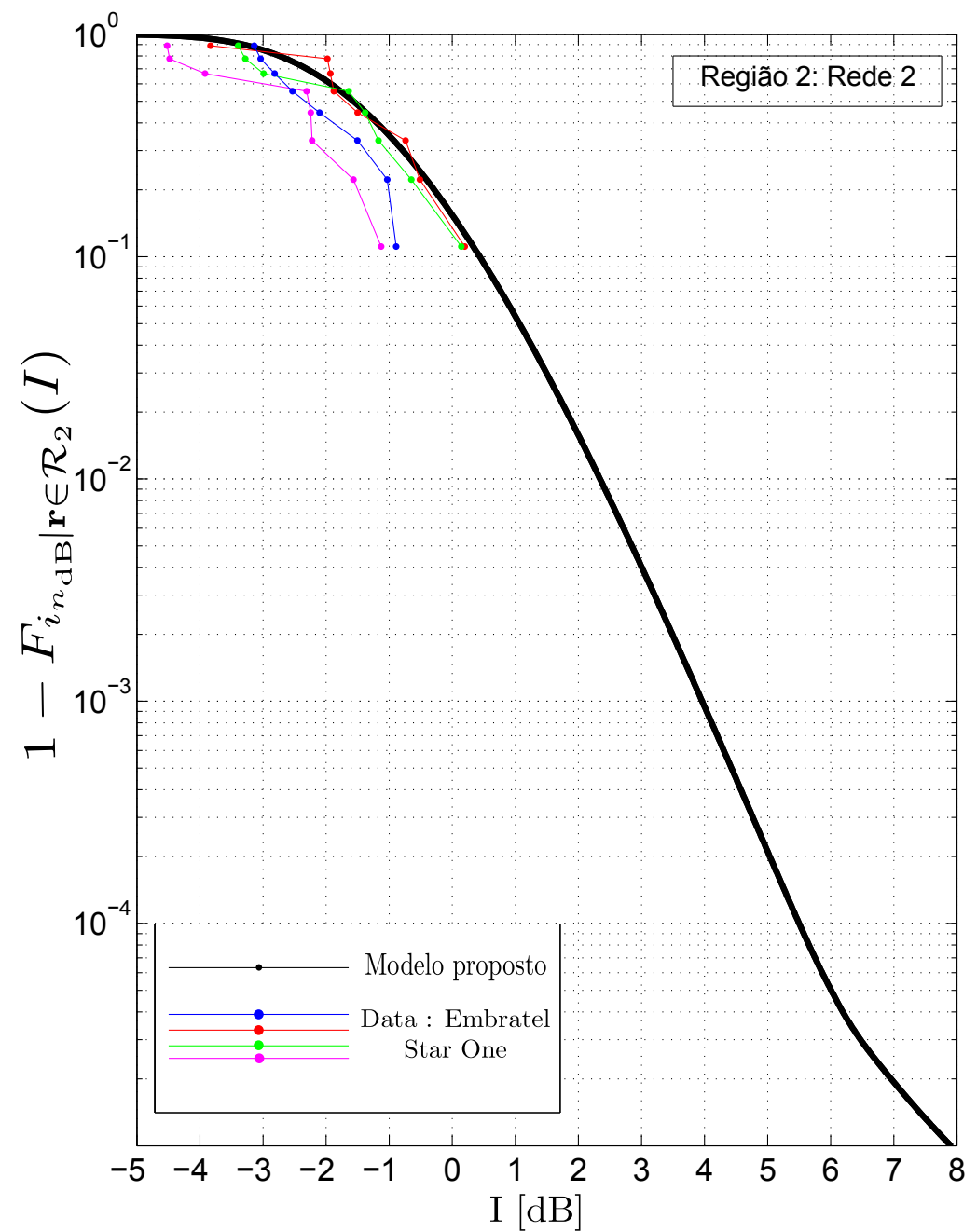

Figura F.8: Número de terminais $\mathcal{N}_{m}: 3$. 
Apêndice F. Comportamento estatístico da Função distribuição de probabilidade complementar correspondente por região $\mathcal{R}_{m},(m=1 \ldots 6)$, para a rede 1 e 2 com $R_{\text {ref }}\left(54,90^{\circ} \mathrm{W}, 9,70^{\circ} \mathrm{S}\right)$

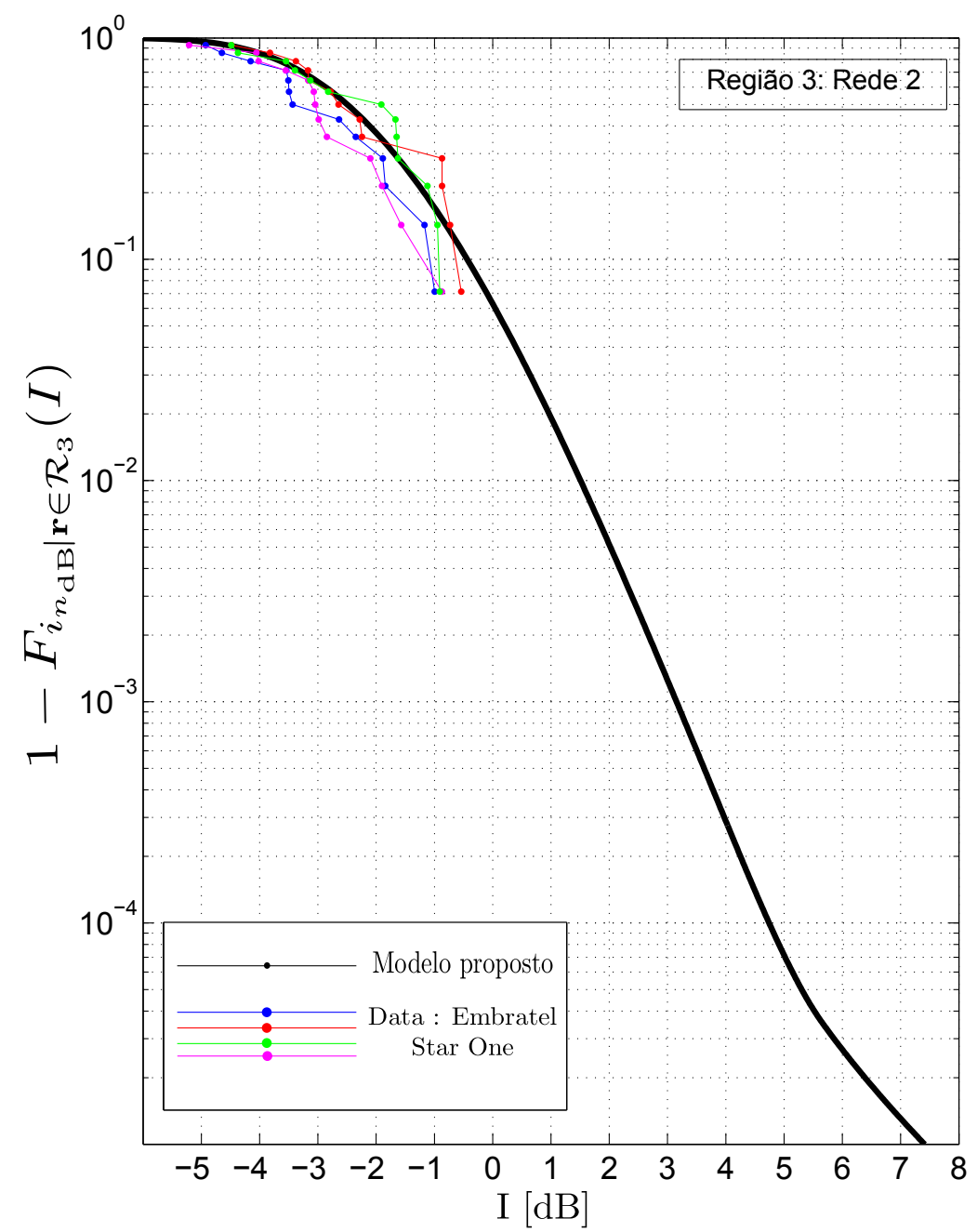

Figura F.9: Número de terminais $\mathcal{N}_{m}: 24$. 
Apêndice F. Comportamento estatístico da Função distribuição de probabilidade complementar correspondente por região $\mathcal{R}_{m},(m=1 \ldots 6)$, para a rede 1 e 2 com $R_{\text {ref }}\left(54,90^{\circ} \mathrm{W}, 9,70^{\circ} \mathrm{S}\right)$

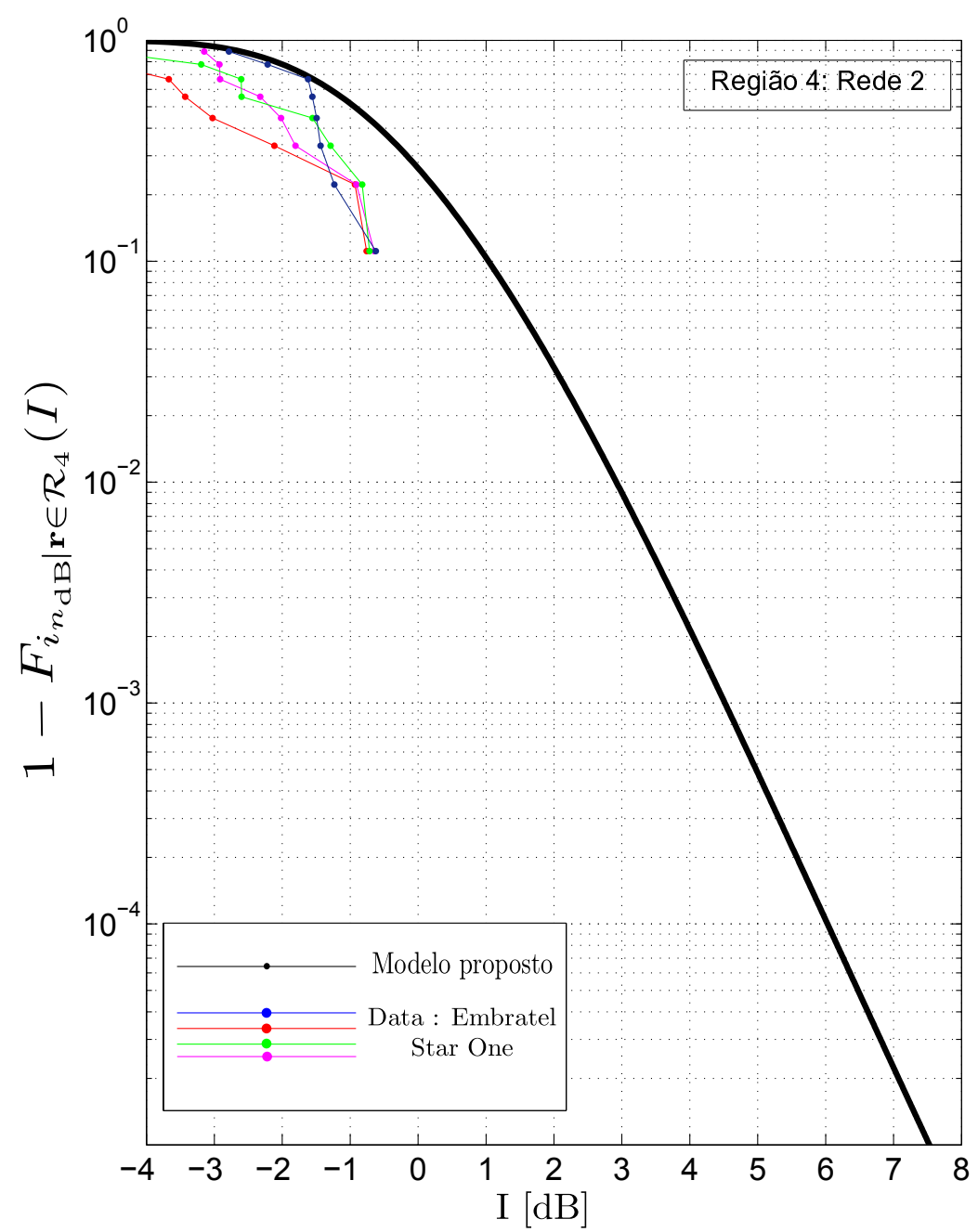

Figura F.10: Número de terminais $\mathcal{N}_{m}: 3$. 
Apêndice F. Comportamento estatístico da Função distribuição de probabilidade complementar correspondente por região $\mathcal{R}_{m},(m=1 \ldots 6)$, para a rede 1 e 2 com $R_{\text {ref }}\left(54,90^{\circ} \mathrm{W}, 9,70^{\circ} \mathrm{S}\right)$

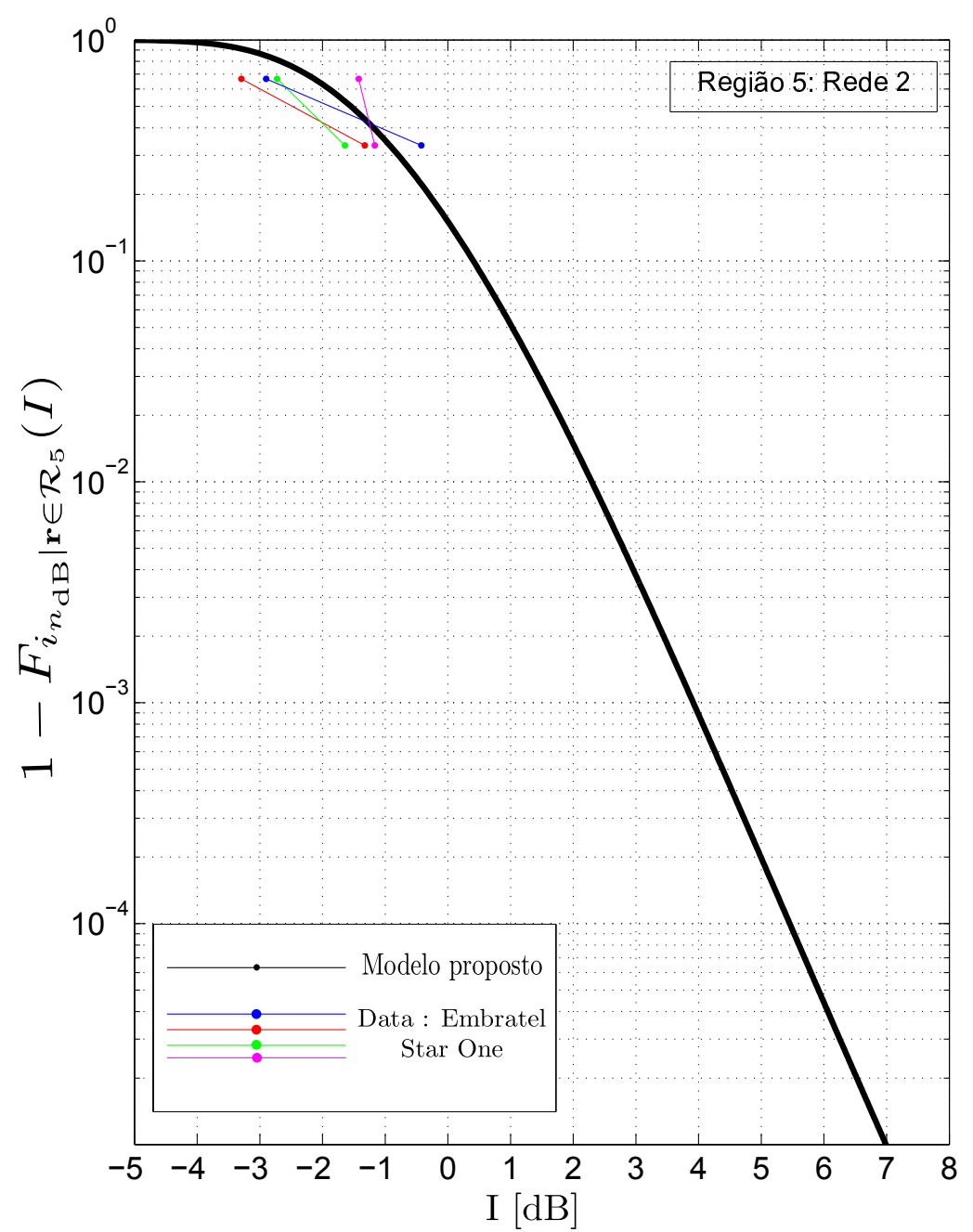

Figura F.11: Número de terminais $\mathcal{N}_{m}: 4$. 
Apêndice F. Comportamento estatístico da Função distribuição de probabilidade complementar correspondente por região $\mathcal{R}_{m},(m=1 \ldots 6)$, para a rede 1 e 2 com $R_{\text {ref }}\left(54,90^{\circ} \mathrm{W}, 9,70^{\circ} \mathrm{S}\right)$

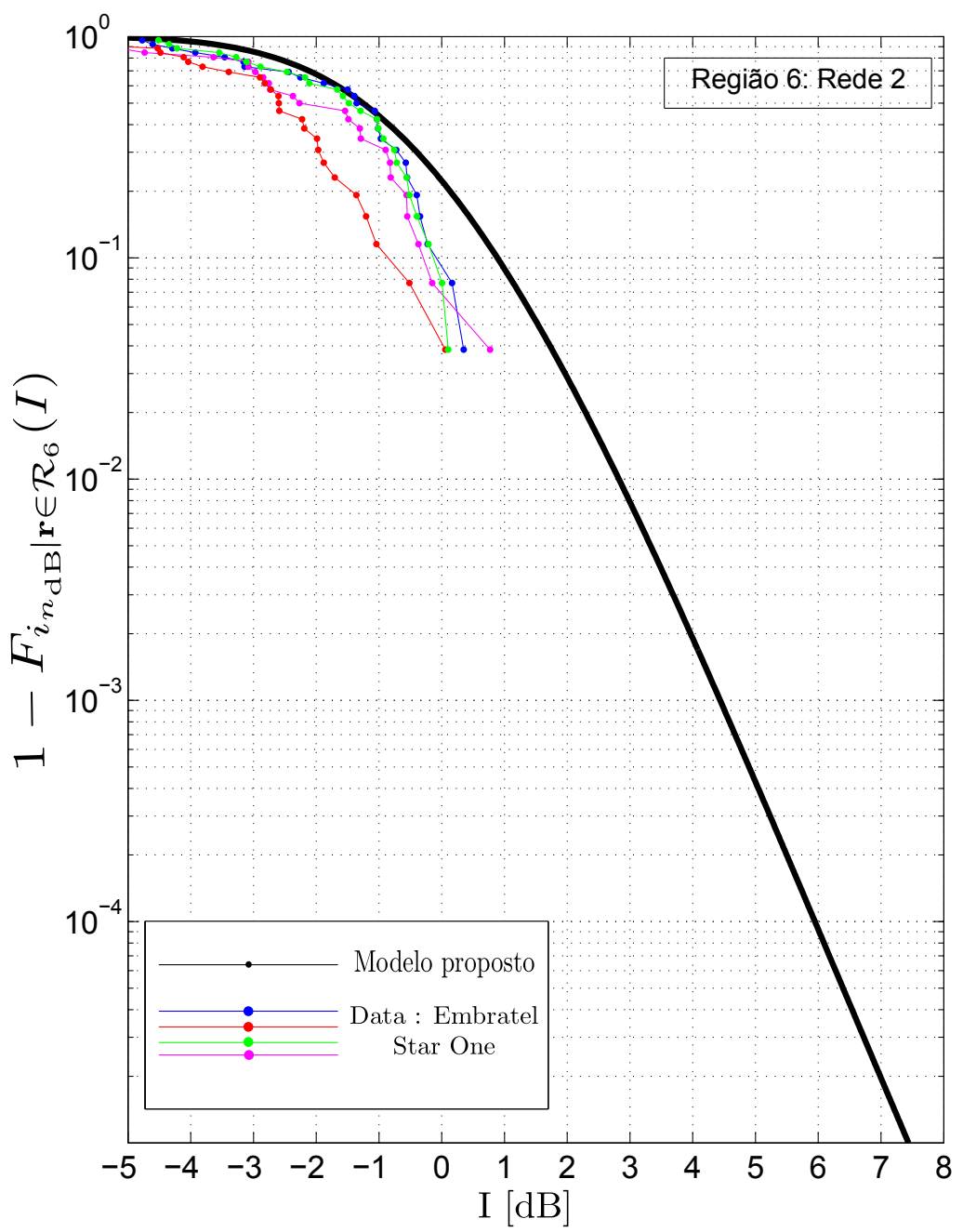

Figura F.12: Número de terminais $\mathcal{N}_{m}: 13$. 